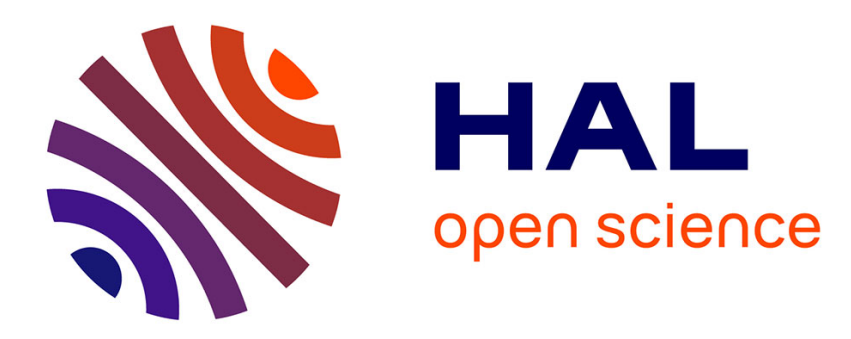

\title{
Utilization of renormalized mean-field theory upon novel quantum materials
}

\author{
Wei-Lin Tu
}

\section{To cite this version:}

Wei-Lin Tu. Utilization of renormalized mean-field theory upon novel quantum materials. General Relativity and Quantum Cosmology [gr-qc]. Université Paul Sabatier - Toulouse III, 2018. English. NNT : 2018TOU30161 . tel-02316080

\section{HAL Id: tel-02316080 \\ https://theses.hal.science/tel-02316080}

Submitted on 15 Oct 2019

HAL is a multi-disciplinary open access archive for the deposit and dissemination of scientific research documents, whether they are published or not. The documents may come from teaching and research institutions in France or abroad, or from public or private research centers.
L'archive ouverte pluridisciplinaire HAL, est destinée au dépôt et à la diffusion de documents scientifiques de niveau recherche, publiés ou non, émanant des établissements d'enseignement et de recherche français ou étrangers, des laboratoires publics ou privés. 


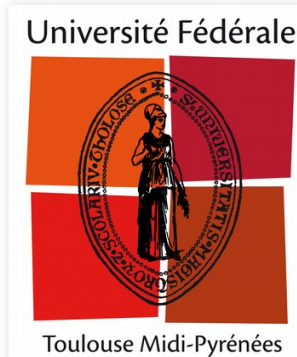

\section{THĖSE}

En vue de l'obtention du

\section{DOCTORAT DE L'UNIVERSITÉ DE TOULOUSE}

Délivré par l'Université Toulouse 3 - Paul Sabatier

Cotutelle internationale : National Taiwan University

$\overline{\text { Présentée et soutenue par }}$

Wei-Lin TU

Le 21 septembre 2018

Théorie de champ moyen renormalisée appliquée aux matériaux quantiques avancés

Ecole doctorale : SDM - SCIENCES DE LA MATIERE - Toulouse

Spécialité : Physique

Unité de recherche :

LPT-IRSAMC - Laboratoire de Physique Théorique

Thèse dirigée par

Didier POILBLANC

Jury

M. Nicolas REGNAULT, Rapporteur

M. Chung-Yu MOU, Rapporteur

M. Chung-Hou CHUNG, Rapporteur

Mme Pina ROMANIELLO, Examinateur

M. Sung-Po CHAO, Examinateur

M. Lei YIN, Examinateur

M. Didier POILBLANC, Directeur de thèse

$M$. Ting-Kuo LEE, Co-directeur de thèse 


\section{Publication Index}

[1] W. Tu and T.K. Lee, "Genesis of charge orders in high temperature superconductors", Scientific Reports 6, 18675 (2016)

Journal: Scientific Reports, Impact factor: 4.259

[2] P. Choubey, W. Tu, T.K. Lee, and P.J. Hirschfeld, "Incommensurate charge ordered states in the $t-t^{\prime}-J$ model”, New Journal Physics 19, 013028 (2017)

Journal: New Journal Physics, Impact factor: 3.786

[3] W. Tu, F. Schindler, T. Neupert, and D. Poilblanc, "Competing orders in the Hofstadter $t$ - $J$ model”, Physical Review B 97, 035154 (2018)

Journal: Physical Review B, Impact factor: 3.836

[4] W. Tu and T.K. Lee, "Pair density waves in the pseudogap phase of cuprate superconductors", manuscript under consideration by Scientific Reports 


\section{Acknowledgements}

To finish a thesis co-advised by two parties is not a easy task. I can never accomplish this without being helped by a lot of people. Among them, first, I need to show my 100 percent gratitude to Dr. T.K. Lee, who granted me this opportunity of conducting research under his supervision. Besides the knowledge I have learned and the training I obtained, the most important spirits I learned from him are the integrity and diligence that a scientist should possess. After occupying a same position and doing the same job for a long time, it is easy for a person to feel exhausted and therefore lose interest. But as a scientist it is a sign of danger because what we are doing for lifetime is the pursuit for the truth and reality. That can be the burden but also the joy. In these days collaborating with Dr. Lee, despite the hard time when we tried to settle down on the same page, I have never saw him lose interest in Physics. Considering how busy he is with also a administrative position as the director of Institute of Physics, Academia Sinica, this is even more difficult to keep the passion like he does. Those spirits I learned from him will be my lifetime treasure no matter if I succeed in becoming a scholar in the future.

Second, Dr. Didier Poilblanc also taught me a lot. The academic environment in Taiwan is not that open compared with Europe where scientists from difference countries can easily meet each other. Thanks for his acceptance of my request of a joint-degree thesis, I got the chance to conduct research in France and therefore obtained many opportunities discussing physical topics with other outstanding scientists. To learn how to express my idea properly 
is crucial in becoming an independent physicist and during the time in France those are among all what I got to learn the most. I think it is fair to say that he led me on the track of becoming an independent researcher from just a apprentice of physics. Thus, I want to thank Didier for not only teaching me a lot of academic knowledge, but also those chances he granted me for interacting with other experts in this field.

Plus, our co-workers mean a lot to me since they were willing to spare time collaborating with us upon certain issues. I would like to thank Dr. Peayush Choubey and Dr. Peter J. Hirschfeld from the University of Florida for participating in the work of analyzing STS results in detail with Wannier function. Dr. Peng-Jen Chen in Institute of Physics, Academia Sinica helped a lot for providing the Wannier90 package, too. Mr. Frank Schindler and Dr. Titus Neupert from the University of Zurich worked with us and provided their beautiful ED results and together we made a nice publication on Physical Review B. Dr. Kenji Harada from the Kyoto University granted me the chance to start a short-term project with him which will become a very useful experience for my career.

As mentioned, to accomplish a co-advised degree is difficult and I cannot go through the administrative process with the help of secretaries from each parties. Therefore I need to thank the secretaries from TK group, Vicky Chen and Judy Hong, and from Department of Physics, NTU, Mr. Jih and Miss Lin. Also Malika Bentour from IRSAMC who provided me a lot of help not only during the administrative process but also for me better fitting in the life in France should be mentioned especially.

Also I want to thank my jury members, Dr. Nicolas Regnault, Dr. Pina Romaniello, Dr. Sung-Po Chao, and Dr. Lei Yin for accepting my request of being included in my committee. I cannot appreciate more for their time and energies spent upon me, a student they maybe never heard of before. Especially, Dr. Chung-Yu Mou and Dr. Nicolas Regnault are also my responsible 
referees who provided their reports to École Doctorale Science de la Matière for my thesis. I would like to thank for their extra effort for me.

Next, all my friends from TK group and Laboratoire de Physique Théorique should be mentioned too. Members from TK group no matter those who already left or still with us provided me help and useful information from time to time. Those routine lunch time became more interesting with your companions. This is also the same for my colleagues in LPT. They helped me adjust myself for the life style in France. Thanks to them I no longer felt alone during the time in abroad. Especially, I would like to thank Mr. HuanKuang $\mathrm{Wu}$ and Dr. Giuseppe Alberti for kindly sharing their experience of preparing a defense.

At last, I need to show my gratitude to my family, my parents Jyy-Jiun Duh and Li-Ching Huang and my sister Jia-Jien Tu, and my girlfriend, Moeka Yamaguchi who we met in France. I would like to thank for your supports during my difficult moments. I want to share the glory, if there is any, to each of you. 


\section{Abstract}

This thesis is aiming in utilizing the strongly correlated $t-J$ Hamiltonian for better understanding the microscopic pictures of certain condensed matter scenario. One of the long existing issues in the Hubbard model and its extreme version, $t-J$ model, lies in the fact that there is not an analytical way of solving them. Therefore, when dealing with these models, numerical approaches become very crucial. In this thesis, we will present one of the methods called renormalized mean-field theory(RMFT) and exploit it upon the $t-J$ model. Thanks to the concept proposed by Gutzwiller, all we have to do is to try to include the correlation of electrons, which is mainly the most difficult part, with several renormalization factors. After obtaining the correct form of these factors, we can apply the routine mean-field theory in solving for the Hamiltonian, which is the principle methodology throughout this thesis.

Next, the physical systems that we are interested in consist of two parts. The mystery of High-Tc superconductivity comes first. After 30 years of its discovery, people still cannot settle down a complete microscopic theory in describing this exotic phenomenon. However, with more and more experimental equipment with higher accuracy nowadays, lots of behavior of copperoxide superconductor(also known as cuprate) have been revealed. Those discoveries can definitely help us better understand its microscopic mechanism. Therefore, from the theoretical side, to compare the calculated data with experiments leads us to know whether our theory is on the right track or not. 
We have produced tons of data and made a decent comparison which will be shown in the main text.

The second system we are curious about is the mechanism of electrons under magnetic field. The Hofstadter butterfly along with its Hamiltonian, the Harper-Hofstadter model has achieved great success in describing free electrons' movement with lattice present. Thus, it will be also interesting to ask the question: what will happen if the electrons are correlated. Our RMFT for $t-J$ Hamiltonian, by adding an additional phase in the hopping term, happens to serve as a great preliminary model for answering this question. We will compare the results of ours with our collaborators, who solved this model by a different approach, the exact diagonalization(ED). Together with our calculations, we proposed several discoveries which might be realized by the cold atom experiments in the future.

Keywords: Strongly correlated systems, $t-J$ model, RMFT 


\section{摘要}

本篇論文致力於使用強關聯電子模型之 $t-J$ 漢密頓量來了解物質 之微觀行為, 關於解釋強關聯之哈伯模型與其之極限對應的 $t-J$ 模型 有一長久以為無法解決的問題為, 我們無法用解析解完美詮釋此量子 模型; 因此, 在探討此模型時數值解便變得極為重要, 在此篇論文中, 我們利用重整化平均場論近似 (RMFT) 之數值理論來探討 $t-J$ 模型的 可能詮釋, 歸功於 Gutzwiller 的發現, 我們將可以把此模型中最困難 之部分：電子的強關聯性重整化為係數置於模型前, 在得到這些相關 係數後, 我們將可以利用平均場論的方法來對角化此一模型以便取得 其本徵函數, 此一方法為此一論文之標準方法。

接下來, 我們關心的物理情境分為兩大類, 第一類為高温超導體之 研究; 在其最初發現於 1987 年以來已經過了 30 年, 但科學家們仍無 法為其定調, 但隨著實驗器具的精準度上升, 我們越來越能清楚得知 其在微觀下的表現, 這有助於幫助理論學家針對其建立一完整模型, 但同時也增加其困難因為要預想一合理之模型能夠完整解釋所有實驗 驗結果並非易事; 我們的計算結果取得了許多數據, 一一與實驗對比 的結果發現雨者之契合度非常之高, 這也是我們對此模型抱有高度信 心之原因, 詳細的比較結果將會在文中一一詳列。

第二類我們感興趣之系統為電子在強磁場作用之下的運動, 霍夫斯 塔德蝴蝶與哈伯一霍夫斯塔德模型在自由電子於強磁場下在晶格内之運 動給了一完整描述, 因此, 探討同樣之運動唯電子具有關聯性便成為 一有趣課題, 而我們的 $t-J$ 模型在動能項增加一相位後, 便成為一個 用來探討此物理情境的可靠模型; 在此一研究之中, 為了能更好得比 
較計算結果, 我們的合作者採用了另一數值方法：完整對角化, 我們 將會比較這雨種方法所計算出之結果並強調其可驗證性於未來之冷原 子實驗中。

關鍵字： 強關聯系統、 $t-J$ 模型、RMFT 


\section{Résumé}

Cette thèse vise à utiliser le $t-J$ Hamiltonian de la corrélation forte pour mieux comprendre la micro-fonctionnalité des scénarios de matériau condensé. Un des problèmes qui existe depuis longtemps est que pour ce type de modèle comme Hubbard Hamiltonian ou $t-J$ Hamiltonian avec une corrélation forte ne peut pas être résolu complètement analytiquement. Par conséquent, quand on aborde ces modèles, il est important de les exploiter de façon numérique. Dans cette thése, nous utiliserons la manière qui s'appelle "Renormalized Mean-Field Theory"(RMFT) pour le $t-J$ Hamiltonian. Grâce à M. Gutzwiller, ce que nous devons faire est simplement de chiffrer des paramètres qui incluent l'influence de la corrélation électronique et de les mettre avant chaque partie du Hamiltonian. Après ce calcul, nous calculerons l'Hamiltonian du champ moyen de manière standard. Ceci sera notre façon principale pour aborder des questions physiques.

Ensuite, nous l'appliquerons sur deux systèmes. Le premier est la mystique de supraconducteur à haute température. Après sa découverte il y a 30 ans, on ne peut pas encore définir une théorie pour expliquer sa micromécanique de manière appropriée. Cependant, avec des équipements avancés, on peut faire des expériences correctement et obtenir des résultats exacts. Ces preuves nous facilitent l'élaboration d'une bonne théorie, même s'il est aussi très difficile d'inclure tous les phénomènes ensemble. Nous avons obtenu des résultats et par rapport aux expériences, ils sont similaires qualitativement. Nous montrerons les détails dans le texte. 
Le deuxième système qui nous intéresse est le mouvement d'électron dans un champ magnétique fort. Le papillon d'Hofstadter et son modèle, l'Hamiltonian de Harper-Hofstadter ont obtenu un grand succès à décrire la mécanique d'électrons libres aux treillis. Donc il est ainsi intéressant de se demander ce qu'il se passera si nous remplaçons des électrons libres avec ceux qui s'interagissent. D'ailleurs, $t-J$ Hamiltonian s'utilise comme bon modèle à le découvrir. Nous allons comparer nos résultats avec ceux de la diagonalisation exacte. Nous proposerons des découvertes intéressantes qui désormais seront réalisées par l'expérience d'atome froide.

Mots clés: Systèmes fortement corrélatives, $t-J$ Hamiltonian, RMFT 


\title{
List of Abbreviations
}

\author{
ABS - Andreev Bound State \\ AF - Antiferromagnetic \\ AP - Anti-Phase
}

AP-CDW - Anti-Phase Charge Density Wave

ARPES - Angle Resolved Photoemission Spectroscopy

ASH - Anderson, Shastry, and Hristopoulos

BdG - Bogoliubov-deGenne

BZ - Brillouin Zone

CB - Checkerboard

CDW - Charge Density Wave

CFP - Commensurate Flux Phase

DC - Discommensurate

DOS - Density Of State

DW - Density Wave

dSC - $d$-Wave Superconductivity

dSC-AFM - $d$-Wave Superconductivity + Antiferromagnetism

ED - Exact Diagonalization

EDC - Energy Distribution Curve

FQH - Fractional Quantum Hall

FLS - Fermi Liquid State

FT - Fourier Transform

GS - Ground State 
GSD - Ground State Degeneracy

GWA - Gutzwiller Approximation

IP - In Phase

IPDW - Incommensurate Pair Density Wave

iPEPS - infinite Projected Entangled-Pair State

LDOS - Local Density Of State

nPDW - nodal Pair Density Wave

PDW - Pair Density Wave

PG - Pseudo-gap

QI-APCDW - Quasi-Incommensurate Anti-Phase Charge Density Wave

RVB - Resonate Valence Bond

RMFT - Renormalized Mean-Field Theory

SC - Superconductivity or Staggered Current

SDW - Spin Density Wave

STS - Scanning Tunneling Spectroscopy

UPOP - Uniform Pairing Order Parameter 


\section{Contents}

\begin{tabular}{lll}
\hline Acknowledgements & v
\end{tabular}

\begin{tabular}{ll} 
Abstract ix & ix \\
\hline
\end{tabular}

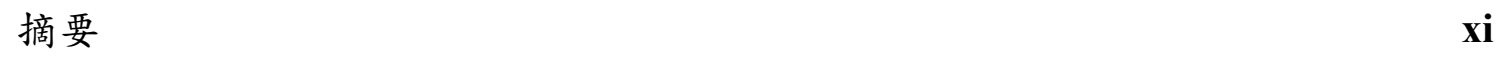

Résumê xiii

\begin{tabular}{ll}
\hline List of Abbreviations & xv
\end{tabular}

\begin{tabular}{lll}
\hline & Introduction & 1
\end{tabular}

1.1 High- $T_{c}$ copper oxide superconductivity $\ldots \ldots \ldots$. . . . . . . . . 1

1.1.1 The density waves . . . . . . . . . . . . . . 3

1.1.2 The pseudo-gap phase . . . . . . . . . . . . 7

1.2 Correlated electrons under strong magnetic field $\ldots \ldots \ldots$. . . . . . 11

\begin{tabular}{|lll}
2 & Renormalized Mean Field Theory & 15
\end{tabular}

$2.1 \quad$ BdG equation of mean-field Hamiltonian $\ldots \ldots \ldots$

2.2 Green's function and LDOS . . . . . . . . . . . . . . . 20

2.3 Spectra weight and many-body Chern number . . . . . . . . . . . 24

\begin{tabular}{|ll|}
\hline 3 & Results I - High $T_{c}$ Cuprate
\end{tabular}

$3.1 \quad$ Real space properties . . . . . . . . . . . . . . . . . . . 27

3.1.1 Charge-ordered patterns . . . . . . . . . . . . 27

3.1 .2 Continuum LDOS . . . . . . . . . . . . . . . 35 
3.1 .3 Bias and doping dependence . . . . . . . . . . . . . . . 37

3.1 .4 Discussion ......................... 41

3.2 Momentum space properties . . . . . . . . . . . . . . . . 42

3.2.1 Particle-hole asymmetry . . . . . . . . . . . . . . . 43

3.2.2 Two-gap in the SC phase . . . . . . . . . . . . . 45

$3.2 .3 \quad$ Finite temperature IPDW states . . . . . . . . . . . . . . 47

3.2 .4 Discussion . . . . . . . . . . . . . . 52

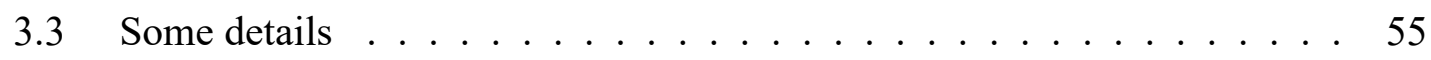

3.3 .1 Method to determine $k_{G} \ldots \ldots \ldots 5$

3.3 .2 Two-gap plots $\ldots \ldots \ldots \ldots 6$

$3.3 .3 \quad$ Choices of $\Gamma \ldots \ldots \ldots \ldots \ldots$

$3.3 .4 \quad$ Fermi arcs and LDOS . . . . . . . . . . . . . 58

4 Results II - Correlated Electrons Under Magnetic Field 61

4.1 Uniform and modulated singlet flux phase . . . . . . . . . . . . . . . . 62

$4.2 \quad$ Fully polarized electron systems $\ldots \ldots \ldots$. . . . . . . . . . . . 68

4.3 Topological properties $\ldots \ldots \ldots$. . . . . . . . . . . . . 69

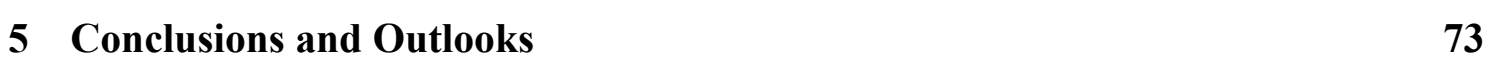

6 Sommaire

$6.1 \quad$ Méthode . . . . . . . . . . . . . . . . . . . . . . 79

6.1.1 Équation BdG de Hamiltonian du champ moyen . . . . . . . . . 79

$6.1 .2 \quad$ Fonctions de Green et LDOS . . . . . . . . . . . . . 85

$6.1 .3 \quad$ La spectre et le nombre de Chern . . . . . . . . . . . . 88

6.2 Supraconduteur à haute température . . . . . . . . . . . . . . . . . 89

6.2.1 Charactéristique en éspace réel . . . . . . . . . . . . . . . 89

$6.2 .2 \quad$ Charactéristique en éspace d'élan . . . . . . . . . . . . . . . 990

6.3 Électrons corrélé dans un champ magnétique . . . . . . . . . . . . 93

6.4 Conclusion . . . . . . . . . . . . . . . . . . . . . . . . . 94 


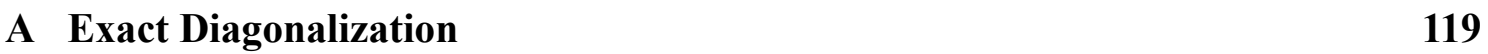

A.1 Model . . . . . . . . . . . . . . . . . . . . . 119

A.2 Many-body Chern number . . . . . . . . . . . . . . . . . . . . . 119

A.3 Results . . . . . . . . . . . . . . . . . . . . . 122

\begin{tabular}{lll}
\hline B Induced Topological-Trivial Transition & 123
\end{tabular} 


\section{List of Figures}

1.1 The phase diagram of hole-doped cuprate. The vertical axis is the temperature in the unit of Kelvin and the horizontal axis is the hole doping level. $T^{*}$ is the transition temperature of pesudo-gap phase, marked by PG while $T_{c}$ is the one for $d$-wave superconductivity, marked by dSC. AF stands for the phase of antiferromagnetism and DW pins out the region where density wave appears. The detailed discussion of each phase is in the text and this figure is borrowed from Ref. [1] . . . . . . . . . . . 3

$2.1 \quad$ Distribution of the phases $\phi_{i j}$ on the bonds of $4 \times 4$ and $2 \times 2$ unit cells (on the 2-torus) for the flux densities $\Phi$ considered in this work(times $\pi / 32$ ). Arrows again indicate the directions of current and negative signs stand for opposite flows. The flux density $\Phi=1 / 4$ has only two different bonds (bond 1 and 2). The right panel shows detailed numbers of variables for

the patterns we have obtained. Those patterns will be discussed later. . .

3.1 Schematic illustration of modulations for stripe like patterns. (a) IP-CDWSDW (b) AP-CDWSDW (c) AP-CDW (d) IP-cCB-sCB (e) AP-cCB-sCB (f) AP-cCB respectively. Size of the circle represents the hole density. The width of the bond around each site represents the amplitude of pairing $\Delta\left(\Delta=\sum_{\mu} \Delta_{\mu}\right)$ and sign is positive (negative) for red (cyan). The size of black arrows represents the spin moment. The average hole density is about 0.1 but 0.09 for IP-cCB-sCB. . . . . . . . . . . . . . . 28 
3.2 (a) Energy per site as a function of hole concentration. Six states are shown in the main figure with notations defined in Table 2. The lower (upper) inset is for stripe (CB) patterns. Blue triangles, circles, and diamonds are for IP-CDW-SDW, AP-CDW-SDW, and AP-CDW respectively. And red triangles, circles and diamonds are for IP-cCB-sCB, AP-cCB-sCB, and AP-cCB respectively. (b) Schematic illustration of modulations for nPDW stripe. The numbers in red denote the hole density at each site while the numbers in black below them represent the pairing amplitude in y direction. The rest numbers above the figure stand for the pairing amplitude in $\mathrm{x}$ direction. Here our pairing amplitudes denote $\left(\left\langle c_{i \uparrow} c_{j \downarrow}\right\rangle\right)$. Note that in this figure neither the size of circles nor the width of bonds represent amplitudes. The hole concentration is 0.125 . (c) LDOS at 8 sites plotted from energy $0.6 \mathrm{t}$ to $-0.6 \mathrm{t}$. The inset shows hole density along the modulation direction of the $n P D W$ stripe and (d) from $0.2 t$ to $-0.2 t$ but shifted vertically for clarity. . . . . . . . . . . . . . . . . . . . . . . . 29

3.3 Properties of nPDW. (a) The real space modulation of nPDW in $32 \times 32$ lattice sites with $\delta=0.125$. Since the pattern repeats itself with an inversion symmetry in the middle bond, here we only show the first 16 sites. The red and black numbers on each bond denote the values of pairing order and the number at each site(black dots) is the hole density. (b)(c) The Fourier transform of the value of hole density(b) and pairing order(c). (d) LDOS of the first 4 sites of this $32 \times 32$ nPDW. (e) Different form factors. . . . . 32

$3.4 \quad$ Figures showing the properties of discommensurate nPDW. (a) The phase variation of this pattern. Site $0-3,12-15$, and 24-27 are of phase equal to $0(2 \pi)$ while sites $6-9,18-21$, and 30-33 are of phase $\pi$. (b) Form factors for discommensurate nPDW. We also include the Fourier transform of hole density(c) and pairing order(d). . . . . . . . . . . . . . . 33 
3.5 (a) Energies of several states chosen by us. Although we have listed ten

different states here, their energies seem to be nearly degenerate and follow the same trend line. (b) Magnitude of $d$ form factor of patterns. Given different states we expect their magnitude to change but still all of them seem to have the same trend: the magnitude maintains the same until doping level exceeds 0.18 , where it starts to drop drastically and becomes zero in the range of $0.18 \sim 0.22 \ldots \ldots \ldots \ldots$

3.6 Continuum LDOS map at $\omega= \pm 0.25 t$ and $\sim 5$ angstrom above BiO plane. (a) LDOS map at $\omega=0.25 t$ in a range of $20 \times 20$ unit cells located in the central region of $60 \times 60$ lattice. (b) Zoomed-in view of the area marked by square in (a). Black dots and open circles represent positions of $\mathrm{Cu}$ and $\mathrm{O}$ atoms, respectively, in the $\mathrm{CuO}$ plane underneath. (c) LDOS map at $\omega=-0.25 t$ in the same region as in (b). . . . . . . . . . . . 36

3.7 Continuum LDOS spectrum registered above $\mathrm{Cu}, O_{x}$ and $O_{y}$ sites in the unit cell $(25,25)$ at an height $\sim 5$ angstrom above $\mathrm{BiO}$ plane (a)without, and (b)with $\Gamma=\alpha|\omega|$ inelastic scattering ( $\alpha=0.25)$, as extracted in [2]. The location of the unit cell can be referred from figure 4(b) as shown in the inset. Dots and open circles represent $\mathrm{Cu}$ and $\mathrm{O}$ atoms, respectively. .

3.8 (a) Bias dependence of the intra-unit cell form factors at $x=0.125$ computed from atomic sublattice averages as described in the text.Next to it are the doping dependence of (a) energy at which d-form factor peaks $\left(\Omega_{d}\right)$ and (b) corresponding magnitude $\left(D_{\max }^{Z}\right) . \ldots \ldots \ldots$

3.9 (a) Bias dependence of average spatial phase difference defined in equation (18). (b) Bias $\Omega_{p}$ at which initial $\pi$ phase jump in $\Delta_{\pi}$ takes place versus doping. (c) Lattice LDOS in the case when nPDW charge and bond modulations are turned off keeping only pair field modulations. (d) Lattice LDOS in the case when nPDW pair field modulations are turned off keeping charge and bond modulations. . . . . . . . . . . . . . . . . 39 
3.10 (a) and (c) Form factors and average spatial phase difference $(\Delta \phi)$ in the case when nPDW charge and bond modulations are turned off keeping only pair field modulations. (b) and (d) Form factors and average spatial phase difference $(\Delta \phi)$, respectively, in the case when nPDW pair field modulations are turned off keeping charge and bond modulations. . . . . 40

3.11 The quasiparticle spectra of a nPDW state calculated in a $32 \times 32$ lattice for hole concentration 0.125: (a) the vertical cuts $(V 1-V 5)$ denote the $y$ component of the momentums scanned from (b)(near nodal region) to (f)(anti-nodal region). (b)-(f): quasiparticle spectra weight for each cut as a function of $k_{y}$ with a fixed $k_{x}$ value shown above each figure. . . . .

3.12 The quasiparticle spectra of a nPDW state calculated in a $32 \times 32$ lattice for hole concentration 0.125: (a) the vertical cuts $(H 1-H 5)$ denote the y component of the momentums scanned from (b)(near nodal region) to (f)(anti-nodal region). (b)-(f): quasiparticle spectra weight for each cut as a function of $k_{x}$ with a fixed $k_{y}$ value shown above each figure. . . . . . . 44

3.13 The gap value evolving from nodal to antinodal region for nPDW for (a) two different lattice sizes at doping 0.125; (b) different doping levels but same size $(30 \times 30)$. Red, green and purple lines are just guides for the eyes. The black dotted(dashed) line is a plotted pure $d$-wave(antinodal)

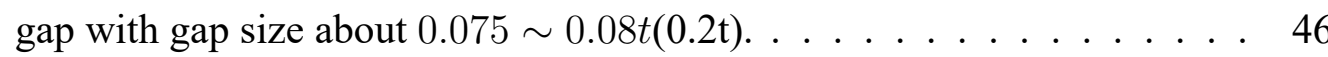

3.14 UPOP vs temperature for $\delta=0.125,0.15$, and $0.16 . T_{p 1}$ and $T_{p 2}$ for each case are marked with different dotted lines of the same colors. The lattice size is $30 \times 30 . \ldots \ldots \ldots \ldots \ldots$. . . . . . . . . . . . . . . . . . . . . 
3.15 Properties of IPDW. (a) The real space modulation of IPDW. The red and black numbers on each bond denote the values of pairing order and the number at each site (black dots) is the hole density. (b) The LDOS for sites near the domain wall(2, 6, 9, 14 in (a)) and in the middle of nearby domain walls(1, 4, 8, 15 in (a)). (c) Different form factors and (d)(e) Fourier transform of hole density(d) and pairing order(e). The red vertical dashed lines mark $|q|=0.5 \pi / a$ corresponding to period $4 a$. Quasiparticle spectra with zero energy in $k$ space for IPDW in $30 \times 30$ lattice sites at $T=0.035 t$ are shown in (f) for $\delta=0.15$. The cyan dotted curve is the Fermi surface of Fermi liquid state with the same doping level. $\Gamma$ used here is equal to $0.25 \sqrt{E^{2}+T^{2}}[2] . \ldots \ldots$. . . . . . . . . . . 49

3.16 (a) Doping dependence of $T_{p 1}$ and $T_{p 2} . T_{p 1} / 2$ and $T_{p 2} / 2$ are shown with the blue triangles and diamonds respectively. The results from NMR [3] are also shown for comparison. We choose $0.1 t \sim 464 K$. (b) The gap values scanned along the Fermi surface at $T=0$ and $2 T_{p 1}$ for $\delta=$ 0.15 . (c) Doping dependence of the relative DOS between IPDW and FLS $\left(D O S_{I P D W} / D O S_{F L S}\right)$ at $T=0.035 t$. The experimental data from [3] for $T=0$ is also plotted for comparison. The inset shows DOS of IPDW vs temperature for $\delta=0.125,0.15$, and 0.16 . $\Gamma$ we used here is $0.25 \sqrt{E^{2}+T^{2}[2]} \ldots \ldots \ldots \ldots \ldots$

3.17 We list several quasi-particle spectra at antinodes $((\pi, 0) /(0, \pi))$ for three different patterns at different temperatures. Although marked as nPDW in the first column, the patterns become IPDW at $T=0.035 t$ and $T=$ $0.05 t$. However their spectra do not change much and the differences of gap values at $(\pi, 0)$ and $(0, \pi)$ are within $10 \%$ [4]. . . . . . . . 52 
3.18 (a) A collection of several data points of $k_{G}-k_{F}$ vs doping at $k_{x}=\pi$. The way of determining the difference of $k_{G}$ and $k_{F}$ is shown in (b): $k_{G}$ determined by examining EDCs plotted from $k_{y}=0$ toward $k_{y}=p i$, for dopant concentration 0.15. $k_{F}$ is determined by Fermi liquid surface and marked along with $k_{G}$ on the EDC plot. The quasiparticle spectra is also shown with Gaussian width $\Gamma=\alpha|E|(\alpha=0.25)$ and marked with positions of $k_{G}$ and $k_{F} \ldots \ldots \ldots \ldots \ldots$

3.19 (a) Two-gap plot for nPDW at $\delta=0.125$ as shown in Fig. 3 in the main text but obtained from different approaches: red line is determined by the gap values shown by quasiparticle spectra but green line comes from EDCs. (b) Relative DOS as a function of hole concentration as in Fig. 3.16(c) in the main text but put together with two different $\Gamma$. The two blue lines are very close to each other. (c) Two gap plots determined by different $\Gamma$ for nPDW at $\delta=0.15$. One can see that these lines nearly overlap with each other. Figure (d) and (e) again show the quasi-particle spectra for nPDW at $\delta=0.125$ (for the $32 \times 32$ lattice) at $k_{x}=0.977 \pi$ but with different $\Gamma:$ (d) $\Gamma=0.01 t$ and (e) $\Gamma=0.25|E|$. Note that in fact (d) is identical as Fig. 3.11(f) in the main text. We can find that although these two figures look quite different due to the choices of $\Gamma$, important features such as location of $k_{G}$ are still the same, only that in (e) the spectra bands are broadened due to larger $\Gamma \ldots \ldots \ldots \ldots$

3.20 (a) and (b) Zero energy quasiparticle spectra in k space before(a) and after(b) taking average of $\mathrm{x}$ - and y-directions PDW. (a) is the same as Fig. $3.15 \mathrm{f}$ and we put it here again for the reason of comparison. Clearly, (b) looks more like the observation by experimental groups. (c) and (d) LDOS at sites near(c) and away from(d) domain walls at different temperatures for $\mathrm{nPDW}(\mathrm{IPDW})$ at $\delta=0.15$. $\Gamma$ used here is equal to $\alpha \sqrt{E^{2}+T^{2}}(\alpha=$ 0.25). All figures shown here are of $30 \times 30$ lattice size. Its $T_{p 1}$ is around

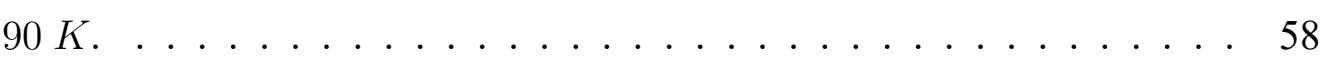


4.1 "Phase diagram" vs electron filling $\rho$ and magnetic flux $\Phi$ showing the various phases presented in Table 4.1. Circles are non-polarized (singlet) states while squares represent ferromagnets. Black symbols correspond to uniform solutions. Red, green, and blue symbols encode symmetrybreaking supercells of size $4 \times 4, \sqrt{2} \times \sqrt{2}$, and $2 \times 2$ (with staggered potential for $\Phi=1 / 4$ ) respectively $\ldots \ldots$. . . . . . . . . . . 63

4.2 Comparison between RMFT and ED energies (per magnetic $4 \times 4$ unit cell). (a) Kinetic energy and (b) magnetic (potential) energy vs inserted flux $\Phi$. The doping level is fixed to $\delta=1 / 8$ and $J=0.3 t$. The numerical values are given in the Table 4.2. . . . . . . . . . . . . . 65

4.3 Schematic patterns and results for the states in this subsection. (a)-(d) show the current and hopping patterns of each state within the $4 \times 4$ sublattice. The widths of the underlying orange bars and black arrows represent the magnitudes of hopping and current on each bond separately. The flows of current are indicated by the arrow directions. The numerical values are shown in Fig. 6.1. . . . . . . . . . . . . . . . . . . 67

4.4 Band structure for the three lowest energy bands for (a) $\nu^{*}=2 / 7$ and (b) $\nu^{*}=2 / 5$. At this doping, the first two bands are filled. Note that in (a) the first two bands are almost degenerate. . . . . . . . . . . . . . . 69

6.1 Distribution of the phases $\phi_{i j}$ on the bonds of $4 \times 4$ and $2 \times 2$ unit cells (on the 2-torus) for the flux densities $\Phi$ considered in this work(times $\pi / 32$ ). Arrows again indicate the directions of current and negative signs stand for opposite flows. The flux density $\Phi=1 / 4$ has only two different bonds (bond 1 and 2). The right panel shows detailed numbers of variables for the patterns we have obtained. Those patterns will be discussed later. . . . 82 
A.1 (a) Vector potential gauge choice for $\Phi=q / 16, q=0, \cdots, 15$. Periodic boundary conditions are assumed. $A_{i j}$ in units of $F=2 \pi \Phi$ is given by the integer number shown between site $i$ and $j$, with positive sign if the respective arrow points from site $i$ to site $j$, and negative sign otherwise.

(b) Spectrum $E(\phi)$ as a function of inserted flux for $\nu=1 / 5$. The Chern number evaluates to 6 , however, there is no indication for a topological GSD . . . . . . . . . . . . . . . . . . . . 120

A.2 Lanczos ED spectrum of $H$ for various values of $\nu$, with $\Phi$ and $\rho$ as given by Table A.1. When there is no magnetization, only the $S_{z}=0, \pm 1$ sector

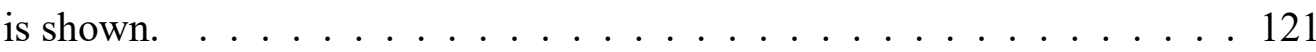

B.1 RMFT energy spectrum as a function of staggered potential $\delta$ with Chern numbers for each band shown beside the figure. For $\Gamma>2 t$ the system is topologically trivial with the Chern number $C$ of the bands zero. At the transition point, the band gap closes and it becomes topologically nontrivial with $C=1$ for the lowest band. After passing the transition point, the gap opens again and the lowest band now possesses a Chern number of -1 . Notice that within this chosen reduced BZ, each of the four bands originating from the $2 \times 2$ modulation is folded into 4 sub-bands, producing a total of 16 bands. . . . . . . . . . . . . . . . . 125 


\section{List of Tables}

3.1 Definition of various nearly degenerate states with respect to the intertwined orders: pair field, charge density, and spin moment. Besides the two uniform solutions, d-wave superconducting(dSC) state and coexistent antiferromagnetic(dSC-AFM) state, all the states to be considered in this paper, unless specifically mentioned, have modulation period $4 a_{0}$ for charge density and bond order. IP(AP) means the pair field is in-phase with period $4 a_{0}$ (anti-phase with period $8 a_{0}$ ). IP has a net pairing order and $\mathrm{AP}$ has none. SDW is the spin density wave with period $8 a_{0}$. $\mathrm{sCB}(\mathrm{cCB})$ denotes the checkerboard pattern of spin(charge) and diag means the diagonal stripe which has in-phase pair field and spin modulation. . . . . . 28 
$4.1 \quad$ Parameter sets used in the following subsections. $N_{\mathrm{S}}, N_{\mathrm{e}}$, and $N_{\Phi}$ are the site, electron and flux numbers used for performing RMFT (those for the ED on a $4 \times 4$ cluster are obtained from a simple rescaling). Sets are listed with decreasing electron filling from top to bottom. The GS is either a singlet $(S=0)$ or fully polarized (FP), i.e., the total spin is $S=\frac{N_{\mathrm{e}}}{2}$ (in that case $\nu^{*}=2 \nu$ is listed and marked with an asterisk). The supercell associated to a possible spontaneous (charge or bond) ordering is also shown. $1 \times 1$ means the GS is uniform. CDW, BDW, and PDW stand for charge, bond, and pairing density wave. SC means staggered current modulation. For $\rho=7 / 16$ and $\Phi=5 / 16$ or $3 / 16$, including (d-wave) superconducting order in addition to CDW/BDW order gives a PDW self-consistent solution with lower energy. For $\rho=1 / 8$ and $\Phi=$ $1 / 4\left(\nu^{*}=1\right)$, the $2 \times 2$ modulation is induced by a staggered potential. Otherwise, translation symmetry breaking (if any) occurs spontaneously. . 62

4.2 Table of the energies and Chern numbers for the self-consistent solutions obtained in RMFT. $E_{0}=E_{\text {kin }}+E_{\text {pot }}$ represents the energy per $4 \times 4$ sublattice. The last column is the Chern number given by summing up the contribution from all the filled (mean-field) bands. The last five rows noted by an asterisk represent the fully polarized states for which $\nu^{*}=2 v$ is listed instead of $\nu \ldots \ldots \ldots \ldots$. . . . . . . . . . . 64

4.3 Summary of the Lanczos exact diagonalization results. . . . . . . . . . . 66

4.4 Table comparing the Chern numbers obtained in the non-interacting case, in the (non-superconducting) RMFT self-consistent solutions and by Lanczos ED. In the two first cases, the Chern numbers are given by summing up the contribution from all the filled bands. The last five rows noted by an asterisk represent the fully polarized states for which $\nu^{*}=2 \nu$ is listed instead of $\nu \ldots \ldots \ldots \ldots \ldots$

A.1 Summary of the Lanczos exact diagonalization results. . . . . . . . . . 121 


\section{Chapter 1}

\section{Introduction}

This introduction is divided into two parts corresponding to two scenarios that we are going to talk about. The first part is for introducing the key issues that still exist and are unsolved to the physical society, which leave the problems of high- $T_{c}$ superconductivity still in the center of stage until now. We will first go through a review upon several important features of it and then bring up the questions that we want to resolve. And then it comes the second interesting system which is the physical system of placing interactive electrons within lattice under strong magnetic field. We will, starting from the initiative by D. R. Hofstadter, compare the calculations executed by two different methods to try to provide a clear picture of what is going to take place when such scenario arrives.

\subsection{High- $T_{c}$ copper oxide superconductivity}

The first discovery of such novel materials with such beautiful characteristic was in 1986 by Bednorz and Müller [5], who won the Nobel Prize in Physics with the Non-stoichiometric copper oxide(also referred to as cuprate), the Lanthanum barium copper oxide $\left(\mathrm{La}_{2-x} \mathrm{Ba}_{x} \mathrm{CuO}\right.$, LBCO) with transition temperature as high as $35 \mathrm{~K}$, in the following year. After the first success, in the years of 1986 to 2008 lots of new cuprate materials were found in series. Among them, the most famous one goes to the yttrium barium copper oxide $\left(\mathrm{YBa}_{2} \mathrm{Cu}_{3} \mathrm{O}_{7}\right.$, YBCO) discovered by Wu and Chu [6] in 1987. Other examples include the bismuth strontium calcium copper oxide $\left(\mathrm{Bi}_{2} \mathrm{Sr}_{2} \mathrm{Ca}_{n} \mathrm{Cu}_{n+1} \mathrm{O}_{2 n+6-d}\right.$, BSCCO) [7] with $\mathrm{T}_{c}=95-107 \mathrm{~K}$ 
varying with the number of $\mathrm{n}$, and thallium barium calcium copper oxide $\left(T l_{m} B a_{2} C a_{n-1} C u_{n} O_{2 n+m+2+\delta}\right.$, TBCCO) with highest possible $T_{c}$ to be $127 \mathrm{~K}$ [8]. Until now, the highest transition temperature confirmed is at $135 \mathrm{~K}$ observed in 1993 with the layered cuprate $\mathrm{HgBa}_{2} \mathrm{Ca}_{2} \mathrm{Cu}_{3} \mathrm{O}_{8+x}$ [9] and when applied under pressure, its $T_{c}$ can achieve above $150 \mathrm{~K}$.

What was so exciting about the discovery of such high- $T_{c}$ cuprate lies on the fact that it breaks the temperature limit set by Bardeen-Cooper-Schrieffer(BCS) theory that was proposed in 1957 [10]. In BCS theory, the phonon plays the role as the medium to combine two electrons, despite their repulsion, in momentum space. In such mechanism, the maximum transition temperature is around $23 \mathrm{~K}$, which is way lower than any temperature at which a efficient industrial usage can be applied. However, the transition temperature of cuprate has surpassed the boiling point of liquid nitrogen, which is easily available nowadays.

Besides the practical application, the violation of the estimation by BCS theory also implies that the phonon interaction may not be enough to properly describe the micromechanism of high- $T_{c}$ phenomena. Thus, one of the questions should be asked naturally will be that what kind of interaction could sustain the electronic pairing stronger than the one mediated by phonon. To answer this, we need to sort out the features. First, we notice that in the phase diagram shown in Figure 1.1, the first appearing phase is the antiferromagnetism(AF). In fact, when there is no doping of hole, the material itself is a Mott insulator [11], composed of the antiferromgnetic ordering and mottism, meaning that the material is an insulator due to the strong electron-electron repulsion. Upon doping, the mottism disappears along with its antiferromagnetic order, which matches the experimental observation of cuprate.

What we want to ask next is the reason why superconductivity appears after the Mottness(antiferromagnetism+mottism) is suppressed by doping. P. W. Anderson was the first among all to propose a theoretical model named after the resonating valence bond theory(RVB) to try to explain the appearance of superconductivity after doping from a insight inspired by strong correlation [12]. In this theory two electrons from neighboring copper atoms tend to form a valence bond. These bonds will resonate within the $\mathrm{Cu}-\mathrm{O}$ 


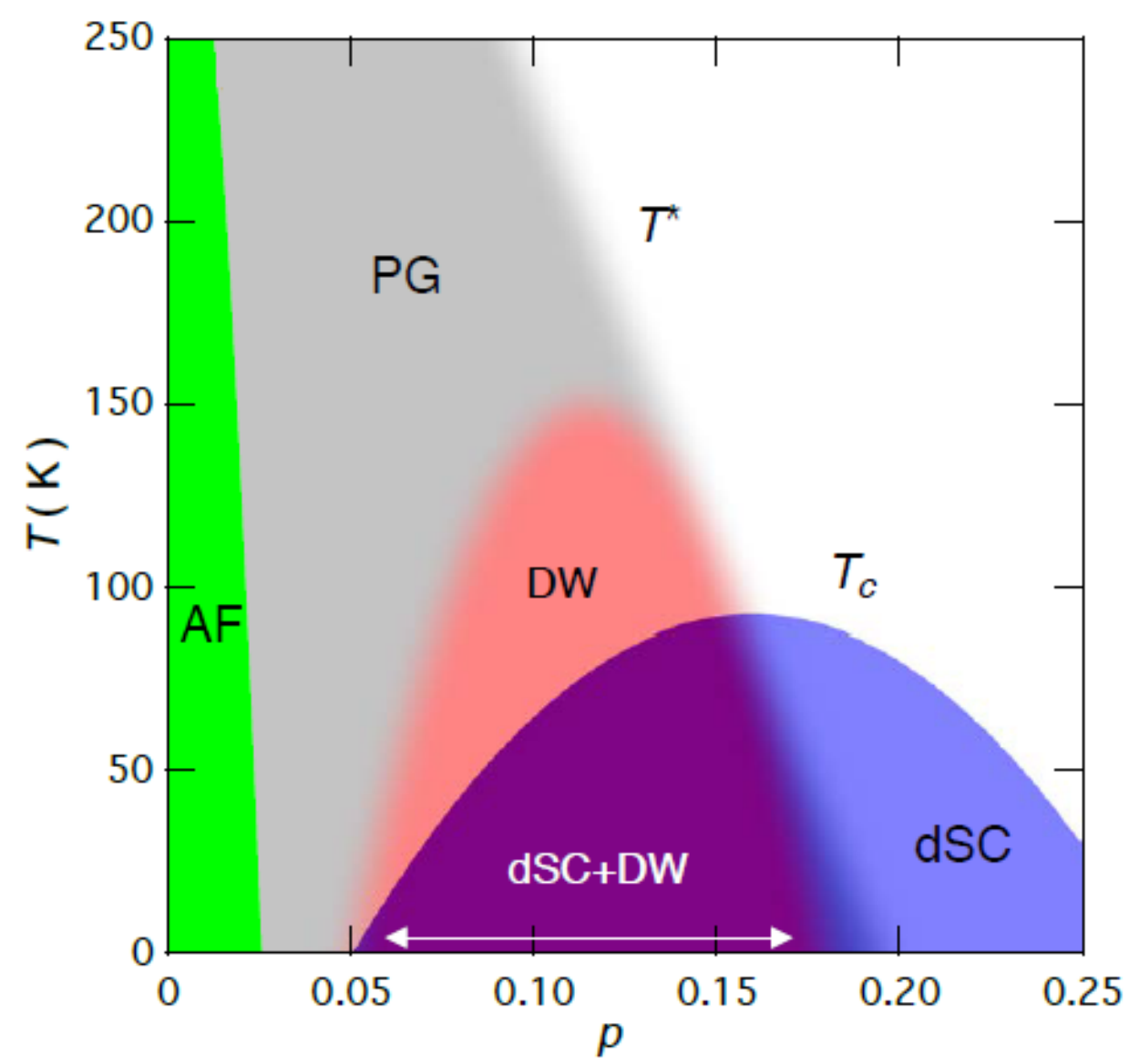

Figure 1.1: The phase diagram of hole-doped cuprate. The vertical axis is the temperature in the unit of Kelvin and the horizontal axis is the hole doping level. $T^{*}$ is the transition temperature of pesudo-gap phase, marked by PG while $T_{c}$ is the one for $d$-wave superconductivity, marked by dSC. AF stands for the phase of antiferromagnetism and DW pins out the region where density wave appears. The detailed discussion of each phase is in the text and this figure is borrowed from Ref. [1]

layer but without doping they cannot transfer in space. However, when vacancies appear with doping, they become mobile and result in the superconductivity. The principle models for describing the RVB theory are the Hubbard and $t-J$ models, and the later will be our central model for this thesis.

\subsubsection{The density waves}

Although the picture provided by Anderson seems to be quite clear and acceptable, the issue of high- $T_{c}$ has not yet been settled down due to several reasons. One, the strong correlated models are usually very difficult to obtain their exact solutions and, unfortunately, the Hubbard and $t-J$ models are of this genre. Despite the effort by physicists 
from numerical parts that provides many reliable calculations for these two models, we still need more proofs before making any further claim. Second, the existence of other unusual phases in the phase diagram is also a pending issue to be explained. The first is the phase of density wave marked by DW in Figure 1.1. Ever since the discovery of the high$T_{c}$ superconductivity, many low-energy charge-ordered states in the cuprate have been discovered. Neutron scattering experiments [13] first emphasized the doping dependence of incommensurate magnetic peaks associated with unidirectional magnetic patterns or stripes. Later, soft X-ray scattering [14] also confirmed the presence of charge orders with these stripes. However, these experiments were performed on the 214 $\left(\mathrm{La}_{2-x} \mathrm{Sr}_{x} \mathrm{CuO}_{4}\right)$ cuprate family. For other cuprate families, the evidence for bond-centered unidirectional domains was found via scanning tunneling spectroscopy [15, 16]. The charge density wave $(\mathrm{CDW})$ order was also found to be induced by the external magnetic field [17]. Recently, more results regrading charge-ordered states [18, 19, 20, 21, 22], and electrondoped cuprates [23] have been reported. The periods of these CDW and their doping dependence are quite different for different cuprate families [22]. In addition to the unidirectional stripe pattern, some experiments have also reported the possible existence of a bidirectional charge-ordered checkerboard pattern [24, 25]. The unidirectional chargeordered states or stripes were found to have a dominant $d$-like symmetry for the intra-unitcell form factor, measured on the two oxygen sites by using the resonant elastic X-ray scattering method [26, 27] and via scanning tunneling spectroscopy(STS) [28]. However, different families seem to prefer different symmetries [26, 27]. In the STS experiments [29], the density waves disappeared above 19\% hole doping. Furthermore, the observation of these CDW states having nodal-like local density of states(LDOS) at low energy but strong spatial variation at high energy in STS [15] strongly implies a new unconventional superconducting state.

The existence of these great varieties of charge-ordered states has created a great debate regarding whether the strong coupling Hubbard model or the $t-J$ model [12] is the proper basic Hamiltonian to describe the cuprates. Many believe that these states "compete" with the superconductivity [30] and that their origin may reveal the fundamental 
understanding of the mechanism of high superconducting temperatures in cuprates. The recent detection of the $d$-form factor at an oxygen site instead of at a $\mathrm{Cu}$ site [26, 27, 28] also raises the question about the suitability of the effective one-band Hubbard or $t-J$ model and the validity of replacing the oxygen hole with a Zhang-Rice singlet [31], which effectively supports a simpler one-band model with $\mathrm{Cu}$ only. Allais et al. [32] proposed that the $d$-symmetry of these form factors, referred to as bond orders [33, 34] because they are measured between the nearest neighbor $\mathrm{Cu}$ bonds, arise from the strong correlation but without other intertwined orders. Furthermore, there are also doubts regarding whether a strong correlation is present or even needed to understand of the superconducting mechanism [35]. However, the complexities of the phase diagram and some recent theoretical works have indicated the possibility of a new phase of matter, i.e., the pair density wave (PDW) [36, 37, 38, 39], as discussed in detail in a recent review article [36]. The new states are considered to have intertwined orders of PDW and CDW or spin density waves (SDW) [36]. Actually there are many different kinds of PDW states that could be either unidirectional [40] or bidirectional like a checkerboard. For the unidirectional PDW state intertwined with CDW and SDW, so called the stripe state, was first proposed by the variational calculation for the $t-J$ model [41]. It could have the same sign of $d$-wave pairing on each site or pairing is in-phase so that the period of modulation of pairing is same as charge density but only half of the SDW. Or it could be the anti-phase stripe having two domains with opposite pairing sign so that the period of pairing modulation is twice of the charge density. The in-phase stripe was later shown [42] to be a stable ground state with half a hole in each period of CDW when a small electron-phonon interaction is included in the $t-J$ model. This half-doped stripe may be what was observed in neutron scattering [13] for the $\operatorname{LBCO}\left(\mathrm{La}_{2-x} \mathrm{Ba}_{x} \mathrm{CuO} \mathrm{O}_{4}\right)$ family.

For quite some time, various calculations [40, 41, 42, 43, 44, 45, 46, 47, 48, 49, 50] on the Hubbard and $t-J$ type models have revealed low-energy intertwined states appearing as stripes or bidirectional charge-ordered states, such as checkerboard(CB). However, these works usually involved different approximations and parameters, which often resulted in different types of charge-ordered patterns, and these studies were mostly con- 
centrated at a hole concentration of $1 / 8$, which is the most notable concentration in early experiments. Hence, it is not clear if these results were the consequence of the invoked assumption or the approximation used, or if they are a generic results in the phase diagrams of cuprates. There were attempts to produce these CDWs or PDWs using a different approach, such as using a mean field theory to study a $t-J$-like model but taking the strong correlation as only a renormalization effect of dispersion [33, 34, 51, 52]. A spin-fluctuation mediated mechanism to produce these states was also proposed for the spin-fermion model [53]. Recently, a novel mechanism of PDW was proposed, i.e., Amperean pairing [39], by using the gauge theory formulation of the resonating-valence-bond picture. In most of these approaches, the wave vectors or periods of the density waves are related to special features of the Fermi surface, including nesting, hot spots or regions with large density of states. However, the opposite doping dependence of CDW periods, observed for 214 and $123\left(\mathrm{YBa}_{2} \mathrm{Cu}_{3} \mathrm{O}_{6+\delta}\right)$ compounds [22], makes the Fermi surface scenario worrisome.

Amid all this confusion, recent numerical progress achieved by using the infinite projected entangled-pair states(iPEPS) method [54], has provided us with a new clue. It was found that the $t-J$ model has several stripe states, with nearly degenerate energy as the uniform state and, with coexistent superconductivity and antiferromagnetism. The period of the PDW moves toward 4 or 5 lattice spacing as $U$ increases and this is more in line with result of the $t-J$ model. When the number of variational parameters is extrapolated to infinity, the authors concluded that the anti-phase stripe, which has no net pairing, has slightly higher energy than the in-phase stripe with a net pairing, which in turn, also has slightly higher energy than the uniform state. The results are quite consistent with the most recent numerical studies on the Hubbard model [55]. They found the stripe states have lower energies than the uniform SC state at $1 / 8$ hole density and for $U=8$ and 12. These results are very consistent with the result of variational Monte Carlo calculations [43] based on the concept of the RVB picture [12]. Furthermore, the results are also consistent with that of renormalized mean-field theory by using a generalised Gutzwiller approximation(GWA) [56] to treat the projection operator in the $t-J$ model [40, 57]. 
Hence, the result provides strong support to more carefully examine the low energy states of the $t-J$ model with the variational approach using GWA.

Among all the discoveries of different states, anti-phase charge density wave (APCDW) and nodal pair density waves (nPDW) have dominant $d$-form factor and exist in the doping range where charge order has been experimentally observed. The AP-CDW is a charge order with commensurate wave vector e.g. $(0,0.25 \pi)$ or $(0.25 \pi, 0)$, that has been studied extensively in Ref. [40]. These states have an accompanying superconducting order parameter that forms domains with opposite signs (AP). The nPDW is an incommensurate charge order with wave vector $(0, Q)$ or $(Q, 0)$, where $Q \sim[0.25 \pi, 0.3 \pi]$. In addition to the modulating component, the pair field has a uniform $d$-wave component giving rise to nodal structure in the density of states at low energies similar to the experimental observation [15]. Thus the nPDW intertwines uniform superconductivity, PDW and charge order. Capello et al. [58] have proposed such a state with an uniform pairing order but it is not a pure $d$-wave order. Instead of proposing a possible state by conjecturing, we have solved a set of self-consistent equations derived from the RMFT. Of the many low energy solutions we found, nPDW explains a number of properties measured by the STS on $\mathrm{BSCCO}\left(\mathrm{Bi}_{2} \mathrm{Sr}_{2} \mathrm{CaCu}_{2} \mathrm{O}_{8+x}\right)$ and $\mathrm{NaCCOC}\left(\mathrm{Ca}_{2-x} \mathrm{Na}_{x} \mathrm{CuO}_{2} \mathrm{Cl}_{2}\right)$ [59]. Its period of the CDW is about half of the PDW. Furthermore, by including the Wannier function in our calculation to take into account the effect of oxygens that were neglected in the simple $t-J$ model, we are able to compute the continuum local density of states of the nPDW. The energy dependence of intra-unit cell form factors and spatial phase variations of these states agrees remarkably well with the STS experiments [28, 59]. We will analyze them further in the following section.

\subsubsection{The pseudo-gap phase}

After demonstrating the appearance of DWs coming from the $t-J$ Hamiltonian with our RMFT, we note that in Figure 1.1 the DW phase is always co-existing with the PG phase. So the next task is to check among those solutions we obtained if some of them are also able to contain the features of PG besides DW. A long standing unresolved puzzle of the 
cuprate high temperature superconductors is the nature of PG phase [60, 61]. Below the PG temperature $T^{*}$ there are experimental evidences of breaking some crystalline symmetry [62, 63]. Breaking of time-reversal symmetry with observation of intra-cell magnetic moments has also been reported [64]. Many more new evidences suggest that this phase should be a nematic phase that breaks the four-fold rotation symmetry of the copper oxygen lattice [65, 66, 67]. In particular there are many reports of the CDW or SDW in the SC and PG phases $[13,14,26,68,69]$. Some of these are likely unidirectional hence without four-fold rotation symmetry. There are experimental evidences indicating the presence of fluctuating or short-range-ordered CDW in the PG phase [19, 26, 70]. Once the CDW sets in and breaks four-fold symmetry [15], the symmetry of pairing order in the SC phase of tetragonal crystal such as $\mathrm{Bi}_{2} \mathrm{Sr}_{2} \mathrm{CaCu}_{2} \mathrm{O}_{8+x}$ should not be expected as a pure $d$-wave as seen in experiments [71, 72]. Thus the formation mechanism of these DWs and its relations with $\mathrm{SC}$ and $\mathrm{PG}$ phases are of great interests.

Before the discovery of these density wave orders in the cuprates, the PG phase has already posed a number of unexplained puzzles. Below a characteristic temperature $T^{*}$ but higher than the SC transition temperature $T_{c}$, the excitation spectra showing a gap was first noticed by the relaxation rate of nuclear magnetic resonance [73] and then by many other transport and spectroscopic measurements [74]. But the most direct observation of this gap structure was shown by the the angle-resolved photoemission spectra(ARPES) [75, 76, 77]. The energy-momentum structure shows an energy gap appears near the boundary, or the antinodal region, of the two-dimensional Brillouin zone(BZ) of the cuprate. However there are four disconnected segments of Fermi surface near the nodal region, or $\left|k_{x}\right|=\left|k_{y}\right|=\pi / 2$. These segments called Fermi arcs have been reported to have their length shrink to zero [78, 79] when extrapolated to zero temperature. There are also results indicating that the arc length is not sensitive to temperature [19, 76]. Then it could also be part of a small pocket [80, 81]. This presence of finite fraction of Fermi surface is consistent with the Knight shift measurement [3] showing a finite density of states(DOS) after the superconductivity is suppressed. The full Fermi surface is recovered either for temperature higher than $T^{*}$ or when doping increases beyond approx- 
imately $19 \%$ as the PG phase disappears. Below $T_{c}$ the gap at antinode merges with the SC gap. Also the ARPES spectra at the antinodal region does not have the usual particlehole symmetry associated with traditional superconductors. This asymmetric antinodal gap onsets at $T^{*}$ and it persists all the way to the SC phase [30, 82].

The phenomena of two gaps, one PG formed above the SC temperature $T_{c}$ and additional SC gap below, and all the exotic behavior associated with it has attracted many attentions as discussed in recent reviews [30, 83]. There are many theoretical proposals devoted to understand the PG as discussed in these review articles [ $[36,60,84]$. But so far it has been difficult to understand the temperature and doping dependence of the Fermi arcs, two gaps and other spectroscopic data, as well as its explicit relationship with the CDW orders and whether any of these are related with the Mott physics or the strong correlation.

However, there are growing evidences that these CDW are not a usual kind but are related to or could be a subsidiary order of the PDW. PDW is in fact a state with spatial modulation of the pairing amplitude and it was first introduced by Larkin and Ovchinnikov [85] and by Fulde and Ferrell [86]. There were quite a number of works proposing that PDW state might be responsible for the many observed exotic phenomena [41, 37, 87, 88, 89, 90] in both SC and PG phases. Many of the works used phenomenological models and weak coupling approaches [51, 53, 91, 92], but some of the numerical works on microscopic models such as the Hubbard model and its low-energy effective $t-J$ model, have found strong evidences for such a state or states. After knowing the importance of PDW and based on the success of the nPDW state to quantitatively explain the real space spectra measured by STS in the SC phase, it naturally leads us to study the spectra in momentum space measured by ARPES.

Instead of concentrating on the microscopic models, the Landau-Ginzburg free energy formalism is used to study the intricate relationship between PDW, CDW, and the uniform pairing order [37, 87, 88, 93, 94]. By including phases of PDW, they could discuss vortex and dislocations as well as the phase diagram. They pointed out that PDW could be responsible for the PG phase. Some of the properties we shall discuss below are consistent with their results; however, they did not consider bond order as an independent field 
whereas we have shown that bond order with dominant intra-unit-cell form factor with $s^{\prime}$ or $d$ symmetry are associated with different PDW states such as stripes or nPDW, respectively, and neither are most of the phenomenological approaches [92, 95]. The work by Lee [39] proposed the Amperean pairing originated from the gauge theory of the RVB picture as the main mechanism for the formation of PDW and it is the dominant order in cuprates. This theory prefers to have bidirectional PDW to have similar gaps at antinodes $(\pi, 0)$ and $(0, \pi)$. They also did not address the issue of bond orders. However, according to our calculation, we are able to demonstrate all the properties mentioned above without any further assumption or experimentally unseen outcome in our states.

In the following content concerning this part, the spectra associated with the nPDW state will be calculated both at $T=0$ and finite temperature with emphasis on the energymomentum dependence of the quasiparticles. The GWA used in the RMFT is considered to be a good approximation at zero temperature. The energy scale imposed by the strong Coulomb repulsion, or Hubbard $U$, is much larger than the scale of room temperature. In addition, both the two main "low" energy scales, $t$ and $J$ about $3000 \sim 4000 \mathrm{~K}$ and $1200 \mathrm{~K}$, respectively, are also much larger. Hence we shall make an assumption that the GWA is reasonably accurate at low but finite temperatures.

After the RMFT is transformed to solve for the self-consistent equations at finite temperatures, we found the average or net uniform pairing order parameter(UPOP) of the nPDWstate decreases to almost zero at a "critical" temperature $T_{p 1}$. This new state still has incommensurate modulations of charge density, pair density and bond orders intertwined, and we shall denote it as incommensurate pair-density-wave(IPDW) state. Just as nPDW state this IPDW state also has the dominant intra-unit-cell $d$-form factors and particle-hole asymmetry for the ARPES spectra [82] at the antinodal region. The major difference with nPDW is the appearance of Fermi arcs and a substantial increase of DOS at Fermi energy but without UPOP. As temperature further increases to $T_{p 2}$, there is no longer a solution of this state. The value of $T_{p 2}$ increases sharply as doping is reduced. The DOS at Fermi energy increases only slightly between $T_{p 1}$ and $T_{p 2}$. The DOS also increases slightly with increasing doping. Comparing these results with experimental data 
on ARPES [30, 82] and DOS deduced from Knight shifts [3], we conclude that it is quite reasonable to take $T_{p 1}$ as the SC transition temperature $T_{c}$ and $T_{p 2}$ as a mean-field version of the PG temperature $T^{*}$ of the copper oxides. These issues will be discussed after the results are presented.

\subsection{Correlated electrons under strong magnetic field}

Next, we will head to discuss the second quantum system that our RMFT of $t-J$ Hamiltonian can be applied for. It is well-known that the Hofstadter butterfly alongside with its Hamiltonian, the Harper-Hofstadter Hamiltonian [96], serves as basis for the study of noninteracting lattice fermions moving in an orbital magnetic field. With the increasing accuracy of experiments, e.g., in laser-manipulated cold atom systems in a two-dimensional square lattice [97, 98, 99, 100, 101, 102], it becomes possible to investigate minute details of this noninteracting model. In addition, cold atom systems have proven to be able to emulate interacting fermionic or bosonic systems [99, 103, 104, 105], which may lead to the realization of exotic material phases such as a cold-atom analog of the fractional quantum Hall(FQH) effect [106], as suggested by promising results from exact diagonalization(ED) of small clusters [107, 108, 109, 110].

Another motivation to study the square lattice in the presence of orbital magnetic fields and strong correlations comes from the field of high- $T_{c}$ superconductivity. The Hubbard Hamiltonian on the square lattice (without external flux) was meant to explain the mechanism of high- $T_{c}$ superconductivity by introducing an on-site interaction $U$, which leads to Mott physics [12]. A $t-J$ Hamiltonian arises from the Hubbard model when the interaction becomes large compared to the bandwidth, with $J=4 t^{2} / U$ being the AF coupling between nearest-neighbor spins (and $t$ being the hopping). In Anderson's original RVB scenario, superconductivity emerges by doping the parent Mott insulator away from halffilling, and proposals for different Mott spin liquid phases have been given. One of them is the Affleck-Marston half-flux state [111, 112, 113], which can be mapped onto free electrons on a lattice with half a magnetic flux quantum per plaquette (and effective hopping $J)$. Away from half-filling, the (mean-field) Affleck-Marston flux phase acquires lowest 
energy density when the flux per unit cell equals exactly the fraction $\nu=\frac{1}{2}(1-\delta)$, where $\delta$ is the doping level [114, 115]. In fact, the corresponding interacting states can be viewed as a Gutzwiller projection of the free fermionic wave functions under magnetic flux. This reveals important aspects of the RVB physics and thus motivates us to perform calculations directly with the $t-J$ Hamiltonian in the presence of an actual external magnetic flux, as we do in the present study.

As mentioned earlier, recently, tensor network studies [54] and density matrix embedding theory [55] provided new evidence that the ground state(GS) of the Hubbard model could indeed be inhomogeneous at finite doping and that its phase diagram shows coexistence of $d$-wave SC order with other instabilities. This fact hinders the possible emergence of topologically nontrivial phases since the latter compete with instabilities. However, in the presence of an external orbital magnetic field, flat bands formed as Landau levels reintroduce this possibility. Also from this perspective it is therefore interesting to consider orbital effects by studying the $t-J$ Hamiltonian in presence of an orbital magnetic field.

For dealing with this issue, here, we will apply two complementary approaches. One is the RMFT. This method, as any mean-field technique, can only detect symmetry-broken phases provided the proper order parameters are introduced by hand, but allows us to reach large system sizes. We compare our results to ED calculations, which are a priori unbiased, but strongly limited in terms of available system sizes. Recently, Gerster et al. [116] demonstrated the existence of a FQH phase akin to the $\nu=1 / 2$ Laughlin state for the spinless bosonic Harper-Hofstadter model by using a tree-tensor network ansatz. This shows that it is possible to obtain novel quantum phases from the Hofstadter Hamiltonian in the presence of interactions and, therefore, provides another motivation to study this model with spinful fermions.

In the following text for this scenario, we will revisit the commensurate flux phase (CFP), which has been studied in previous work [48, 117]. Here, we will in particular focus on charge instabilities and topological features of the CFP. Instabilities toward ferromagnetic phases(fully polarized states) are described next, showing good agreement between our two numerical approaches. Topological aspects (e.g., the computation of Chern 
numbers) and comments on the search for potential FQH physics are later subsumed. 


\section{Chapter 2}

\section{Renormalized Mean Field Theory}

In this section, we will go through the main method of ours in this thesis, the RMFT, in detail starting from the $t-J$ Hamiltonian. We will also demonstrate how we can calculate for some key properties such as LDOS and spectra weight with our BogoliubovdeGenne(BdG) wavefunctions.

\subsection{BdG equation of mean-field Hamiltonian}

In this thesis, we consider the $2 \mathrm{D} t-J$ model, i.e., the large- $U$ limit of the 2D Hubbard model, in an external magnetic field as our interacting Hamiltonian,

$$
\begin{gathered}
H=\underbrace{-\sum_{\langle i, j\rangle, \mu} P_{\mathrm{G}}\left(t_{i j} c_{i \mu}^{\dagger} c_{j \mu}+\text { h.c. }\right) P_{\mathrm{G}}}_{H_{\mathrm{kin}}} \underbrace{\sum_{\langle i, j\rangle} J \boldsymbol{S}_{i} \cdot \boldsymbol{S}_{j}}_{H_{\mathrm{pot}}}, \\
t_{i j}=t e^{\mathrm{i} A_{i j}}=t_{j i}^{*}, \quad \boldsymbol{S}_{i}=\sum_{\mu, \nu} c_{i \mu}^{\dagger} \boldsymbol{\sigma}_{\mu \nu} c_{j \nu},
\end{gathered}
$$

where $c_{i \mu}^{\dagger}\left(c_{i \mu}\right)$ is the creation (annihilation) operator for an electron of spin $\mu=\uparrow, \downarrow$ on lattice site $i$, so that $n_{i \mu}=c_{i \mu}^{\dagger} c_{i \mu}$ is the site number operator per spin, $P_{\mathrm{G}}=\prod_{i}\left(1-n_{i \uparrow} n_{i \downarrow}\right)$ is the Gutzwiller projector onto the Hilbert subspace of at most singly-occupied sites, and $\boldsymbol{\sigma}=\left(\sigma_{x}, \sigma_{y}, \sigma_{z}\right)^{\mathrm{T}}$ is the vector of $2 \times 2$ Pauli spin matrices. In the exact mapping from Hubbard to $t-J$ model there is another term of order $t^{2} / U$, the so called three-site hopping, which describes hopping of singlet pairs. This term has been shown to have no influence 
on the mean-field phase diagram [118] and is therefore excluded in our work. The AF coupling $J$ is chosen to be equal to 0.3 times the hopping $t$ throughout the thesis.

The magnetic field enters via the phases $A_{i j}=\int_{i}^{j} \boldsymbol{A}(\boldsymbol{x}) \cdot d \boldsymbol{x}$, where the vector potential $\boldsymbol{A}(\boldsymbol{x})$ is defined by the relation $\boldsymbol{B}(\boldsymbol{x})=\nabla \times \boldsymbol{A}(\boldsymbol{x})$, corresponding to a flux per plaquette $F=\int_{\square} \boldsymbol{B}(\boldsymbol{x}) \cdot d \boldsymbol{\Sigma}=A_{i, i+\hat{x}}+A_{i+\hat{x}, i+\hat{x}+\hat{y}}+A_{i+\hat{x}+\hat{y}, i+\hat{y}}+A_{i+\hat{y}, i}$, which we take to be independent of $i$. Here we choose $F=2 \pi \Phi$, with $\Phi$ given by fractions such as $\frac{7}{16}, \frac{5}{16}$, etc. Note that since we work in units where $h=e=1, \Phi=1$ corresponds to one magnetic flux quantum. $A_{i j}=0$ when dealing with the cuprate problem in this thesis.

The standard procedure of RMFT is to first replace the Gutzwiller projection operator by renormalized factors $g^{t}$ and $g^{s}$ so that

$$
\begin{aligned}
& \left\langle\Psi\left|c_{i \mu}^{\dagger} c_{j \mu}\right| \Psi\right\rangle=g_{i j \mu}^{t}\left\langle\Psi_{0}\left|c_{i \mu}^{\dagger} c_{j \mu}\right| \Psi_{0}\right\rangle, \\
& \left\langle\Psi\left|\boldsymbol{S}_{i} \cdot \boldsymbol{S}_{j}\right| \Psi\right\rangle=g_{i j}^{s}\left\langle\Psi_{0}\left|\boldsymbol{S}_{i} \cdot \boldsymbol{S}_{j}\right| \Psi_{0}\right\rangle
\end{aligned}
$$

where $\left|\Psi_{0}\right\rangle$ is the un-projected wavefunction and $|\Psi\rangle=P_{\mathrm{G}}\left|\Psi_{0}\right\rangle$. The Hamiltonian then becomes:

$$
\begin{aligned}
H= & -\sum_{\langle i, j\rangle \mu} g_{i j \mu}^{t} t_{i j} e^{i A_{i j}}\left(c_{i \mu}^{\dagger} c_{j \mu}+\text { h.c. }\right) \\
& +\sum_{\langle i, j\rangle} J\left[g_{i j}^{s, z} S_{i}^{s, z} S_{j}^{s, z}+g_{i j}^{s, x y}\left(\frac{S_{i}^{+} S_{j}^{-}+S_{i}^{-} S_{j}^{+}}{2}\right)\right]
\end{aligned}
$$

where $g_{i j \sigma}^{t}, g_{i j}^{s, z}$, and $g_{i j}^{s, x y}$ are the Gutzwiller factors, which depend on the values of the pairing field $\Delta_{i j \mu}^{v}$, bond order $\chi_{i j \mu}^{v}$, spin moment $m_{i}^{v}$, and hole density $\delta_{i}$ :

$$
\begin{aligned}
& m_{i}^{v}=\left\langle\Psi_{0}\left|S_{i}^{z}\right| \Psi_{0}\right\rangle \\
& \Delta_{i j \mu}^{v}=\mu\left\langle\Psi_{0}\left|c_{i \mu} c_{j \bar{\mu}}\right| \Psi_{0}\right\rangle \\
& \chi_{i j \mu}^{v}=\left\langle\Psi_{0}\left|c_{i \mu}^{\dagger} c_{j \mu}\right| \Psi_{0}\right\rangle \\
& \delta_{i}=1-\left\langle\Psi_{0}\left|n_{i}\right| \Psi_{0}\right\rangle
\end{aligned}
$$

where $\left|\Psi_{0}\right\rangle$ is the unprojected wavefunction. The superscript $v$ is used to denote that these quantities are variational parameters instead of real physical quantities. As for the phases 

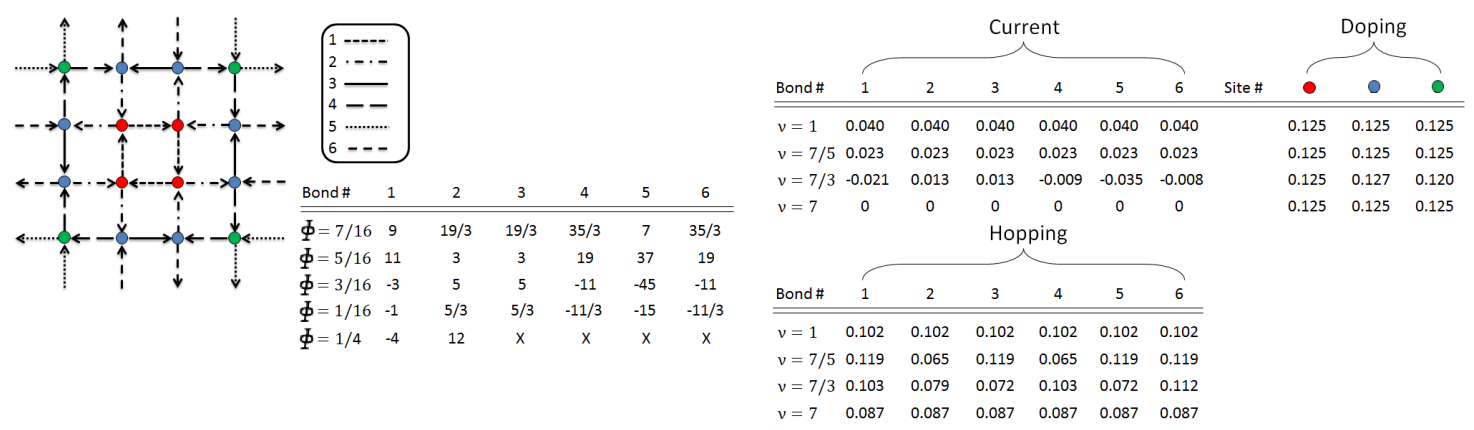

Figure 2.1: Distribution of the phases $\phi_{i j}$ on the bonds of $4 \times 4$ and $2 \times 2$ unit cells (on the 2-torus) for the flux densities $\Phi$ considered in this work(times $\pi / 32$ ). Arrows again indicate the directions of current and negative signs stand for opposite flows. The flux density $\Phi=1 / 4$ has only two different bonds (bond 1 and 2). The right panel shows detailed numbers of variables for the patterns we have obtained. Those patterns will be discussed later.

$\left(A_{i j}\right)$, we followed Ref. [117]. The numbers for different flux per plaquette $\Phi$ are shown in Fig. 6.1. We will start by considering the Gutzwiller factors first proposed by Ogata and Himeda [41, 45], which are given by

$$
\begin{aligned}
& g_{i j \mu}^{t}=g_{i \mu}^{t} g_{j \mu}^{t} \\
& g_{i \mu}^{t}=\sqrt{\frac{2 \delta_{i}\left(1-\delta_{i}\right)}{1-\delta_{i}^{2}+4\left(m_{i}^{v}\right)^{2}} \frac{1+\delta_{i}+\mu 2 m_{i}^{v}}{1+\delta_{i}-\mu 2 m_{i}^{v}}} \\
& g_{i j}^{s, x y}=g_{i}^{s, x y} g_{j}^{s, x y} \\
& g_{i}^{s, x y}=\frac{2\left(1-\delta_{i}\right)}{1-\delta_{i}^{2}+4\left(m_{i}^{v}\right)^{2}} \\
& g_{i j}^{s, z}=g_{i j}^{s, x y} \frac{2\left(\left(\bar{\Delta}_{i j}^{v}\right)^{2}+\left(\bar{\chi}_{i j}^{v}\right)^{2}\right)-4 m_{i}^{v} m_{j}^{v} X_{i j}^{2}}{2\left(\left(\bar{\Delta}_{i j}^{v}\right)^{2}+\left(\bar{\chi}_{i j}^{v}\right)^{2}\right)-4 m_{i}^{v} m_{j}^{v}} \\
& X_{i j}=1+\frac{12\left(1-\delta_{i}\right)\left(1-\delta_{j}\right)\left(\left(\bar{\Delta}_{i j}^{v}\right)^{2}+\left(\bar{\chi}_{i j}^{v}\right)^{2}\right)}{\sqrt{\left(1-\delta_{i}^{2}+4\left(m_{i}^{v}\right)^{2}\right)\left(1-\delta_{j}^{2}+4\left(m_{j}^{v}\right)^{2}\right)}}
\end{aligned}
$$

where $\bar{\Delta}_{i j}^{v}=\sum_{\mu} \Delta_{i j \mu}^{v} / 2$ and $\bar{\chi}_{i j}^{v}=\sum_{\mu} \chi_{i j \mu}^{v} / 2$. In the presence of AF, $\Delta_{i j \uparrow}^{v} \neq \Delta_{i j \downarrow}^{v}$. For singlet states the magnetization $m_{i}^{v}$ is equal to zero and $n_{i \uparrow}=n_{i \downarrow}=\frac{1}{2}\left(1-\delta_{i}\right)$. However, for the fully polarized scenario $m_{i}^{v}=n_{i \uparrow} / 2$ while $n_{i \uparrow}=\left(1-\delta_{i}\right), n_{i \downarrow}=0$, where we assume that all electrons have spin up. This set of Gutzwiller factors corresponds to finite doping and is consistent with variational Monte Carlo calculations [41, 45].

After we replace the projection operator by the Gutzwiller factors by using the mean- 
field order parameters defined in Eq. 6.4, the energy of the renormalized Hamiltonian(Eq. 6.3) becomes the following as we part the four operators with mean-field variables:

$$
\begin{aligned}
E=\left\langle\Psi_{0}|H| \Psi_{0}\right\rangle= & -\sum_{i, j, \mu} g_{i j \mu}^{t} t e^{i A_{i j}}\left(\chi_{i j \mu}^{v}+\text { h.c. }\right) \\
& -\sum_{\langle i, j\rangle \mu} J\left(\frac{g_{i j}^{s, z}}{4}+\frac{g_{i j}^{s, x y}}{2} \frac{\Delta_{i j \bar{\mu}}^{v *}}{\Delta_{i j \mu}^{v *}}\right) \Delta_{i j \mu}^{v *} \Delta_{i j \mu}^{v} \\
& -\sum_{\langle i, j\rangle \mu} J\left(\frac{g_{i j}^{s, z}}{4}+\frac{g_{i j}^{s, x y}}{2} \frac{\chi_{i j \bar{\mu}}^{v *}}{\chi_{i j \mu}^{v *}}\right) \chi_{i j \mu}^{v *} \chi_{i j \mu}^{v} \\
& +\sum_{\langle i, j\rangle} g_{i j}^{s, z} J m_{i}^{v} m_{j}^{v}
\end{aligned}
$$

Next we want to minimize the energy under two constraints: $\sum_{i} n_{i}=N_{\mathrm{e}}$ and $\left\langle\Psi_{0} \mid \Psi_{0}\right\rangle=$ 1. Thus our cost function to be minimized is

$$
W=\left\langle\Psi_{0}|H| \Psi_{0}\right\rangle-\lambda\left(\left\langle\Psi_{0} \mid \Psi_{0}\right\rangle-1\right)-\epsilon\left(\sum_{i} n_{i}-N_{\mathrm{e}}\right)
$$

The mean-field Hamiltonian becomes

$$
H_{\mathrm{MF}}=\sum_{\langle i, j\rangle \mu} \frac{\partial W}{\partial \chi_{i j \mu}^{v}} c_{i \mu}^{\dagger} c_{j \mu}+\text { h.c. }+\sum_{\langle i, j\rangle \mu} \frac{\partial W}{\partial \Delta_{i j \mu}^{v}} \mu c_{i \mu} c_{j \bar{\mu}}+\text { h.c. }+\sum_{i, \mu} \frac{\partial W}{\partial n_{i \mu}} n_{i \mu}
$$

Eq. (6.8) satisfies the Schrödinger equation $H_{\mathrm{MF}}\left|\Psi_{0}\right\rangle=\lambda\left|\Psi_{0}\right\rangle$. The three derivatives are defined as

$$
\begin{aligned}
& H_{i j \mu}=\frac{\partial W}{\partial \chi_{i j \mu}^{v}}=-J\left(\frac{g_{i j}^{s, z}}{4}+\frac{g_{i j}^{s, x y}}{2} \frac{\chi_{i j \mu}^{v *}}{\chi_{i j \mu}^{v *}}\right) \chi_{i j \mu}^{v *}-g_{i j \mu}^{t} t_{i j} e^{i A_{i j}}+\frac{\partial W}{\partial g_{i j}^{s, z}} \frac{\partial g_{i j}^{s, z}}{\partial \chi_{i j \mu}^{v}} \\
& D_{i j}^{*}=\frac{\partial W}{\partial \Delta_{i j \uparrow}^{v}}=-J\left(\frac{g_{i j}^{s, z}}{4}+\frac{g_{i j}^{s, x y}}{2} \frac{\Delta_{i j \downarrow}^{v *}}{\Delta_{i j \uparrow}^{v *}}\right) \Delta_{i j \uparrow}^{v *}+\frac{\partial W}{\partial g_{i j}^{s, z}} \frac{\partial g_{i j}^{s, z}}{\partial \Delta_{i j \uparrow}^{v}}
\end{aligned}
$$




$$
\epsilon_{i \mu}=-\frac{\partial W}{\partial n_{i \mu}}=\epsilon-\sum_{j} \frac{\partial W}{\partial g_{i j}^{s, x y}} \frac{\partial g_{i j}^{s, x y}}{\partial n_{i \mu}}-\sum_{j} \frac{\partial W}{\partial g_{i j}^{s, z}} \frac{\partial g_{i j}^{s, z}}{\partial n_{i \mu}}-\sum_{j \mu^{\prime}} \frac{\partial W}{\partial g_{i j \mu^{\prime}}^{t}} \frac{\partial g_{i j \mu^{\prime}}^{t}}{\partial n_{i \mu}}
$$

Eq. (6.10) is the effective local chemical potential. $H_{\mathrm{MF}}$ can be rewritten in form of the BdG equations

$$
H_{\mathrm{MF}}=\left(c_{i \uparrow}^{\dagger}, c_{i \downarrow}\right)\left(\begin{array}{cc}
H_{i j \uparrow} & D_{i j} \\
D_{j i}^{*} & -H_{j i \downarrow}
\end{array}\right)\left(\begin{array}{c}
c_{j \uparrow} \\
c_{j \downarrow}^{\dagger}
\end{array}\right)
$$

We can diagonalize $H_{\mathrm{MF}}$ to obtain an equal number of positive and negative eigenvalues together with their corresponding eigenvectors $\left(u_{i}^{n}, v_{i}^{n}\right)$. With these eigenvectors, we can determine the order parameters at zero temperature by following equations

$$
\begin{aligned}
& n_{i \uparrow}=\left\langle c_{i \uparrow}^{\dagger} c_{i \uparrow}\right\rangle=\sum_{n}\left|u_{i}^{n}\right|^{2} f\left(E_{n}\right) \\
& n_{i \downarrow}=\left\langle c_{i \downarrow}^{\dagger} c_{i \downarrow}\right\rangle=\sum_{n}\left|v_{i}^{n}\right|^{2}\left(1-f\left(E_{n}\right)\right) \\
& \Delta_{i j \uparrow}^{v}=\left\langle c_{i \uparrow} c_{j \downarrow}\right\rangle=\sum_{n_{+}} u_{i}^{n} v_{j}^{n *}\left(1-f\left(E_{n}\right)\right)-u_{j}^{n} v_{i}^{n *}\left(1-f\left(-E_{n}\right)\right) \\
& \Delta_{i j \downarrow}^{v}=-\left\langle c_{i \downarrow} c_{j \uparrow}\right\rangle=\sum_{n_{+}} u_{j}^{n} v_{i}^{n *}\left(1-f\left(E_{n}\right)\right)-u_{i}^{n} v_{j}^{n *}\left(1-f\left(-E_{n}\right)\right) \\
& \chi_{i j \uparrow}^{v}=\left\langle c_{i \uparrow}^{\dagger} c_{j \uparrow}\right\rangle=\sum_{n} u_{j}^{n} u_{i}^{n *} f\left(E_{n}\right) \\
& \chi_{i j \downarrow}^{v}=\left\langle c_{i \downarrow}^{\dagger} c_{j \downarrow}\right\rangle=\sum_{n} v_{i}^{n} v_{j}^{n *}\left(1-f\left(E_{n}\right)\right)
\end{aligned}
$$

The sum for $n_{+}$means the set of eigenvectors with positive energies. $f\left(E_{n}\right)$ is the FermiDirac distribution:

$$
f\left(E_{n}\right)=\frac{1}{e^{E_{n} / T}+1}
$$

An iterative method is used to solve $H_{M F}$ self-consistently. The convergence is achieved for every order parameter if its value changes less than $10^{-3}$ between successive iterations or sometimes $10^{-4}$ if we demand a higher accuracy. After the self-consistency is achieved, 
we can calculate order parameters and their formula are:

$$
\begin{aligned}
\Delta_{i} & =\sum_{\mu}\left(g_{i, \mu}^{t} g_{i+\hat{x}, \bar{\mu}}^{t} \Delta_{i, i+\hat{x}, \mu}^{v}+g_{i, \mu}^{t} g_{i-\hat{x}, \bar{\mu}}^{t} \Delta_{i, i-\hat{x}, \mu}^{v}-g_{i, \mu}^{t} g_{i+\hat{y}, \bar{\mu}}^{t} \Delta_{i, i+\hat{y}, \mu}^{v}-g_{i, \mu}^{t} g_{i-\hat{y}, \bar{\mu}}^{t} \Delta_{i, i-\hat{y}, \mu}^{v}\right) / 8, \\
m_{i} & =\left(\sqrt{g_{i, i+\hat{x}}^{s, z}}+\sqrt{g_{i, i-\hat{x}}^{s, z}}+\sqrt{g_{i, i+\hat{y}}^{s, z}}+\sqrt{g_{i, i-\hat{y}}^{s, z}}\right) m_{i}^{v} / 4, \\
K_{i, i+\hat{x}} & =\frac{1}{2} \sum_{\mu} g_{i, i+\hat{x}, \mu}^{t}\left\langle c_{i \mu}^{\dagger} c_{i+\hat{x} \mu}\right\rangle+g_{i+\hat{x}, i, \mu}^{t}\left\langle c_{i+\hat{x} \mu}^{\dagger} c_{i \mu}\right\rangle, \\
K_{i, i+\hat{y}} & =\frac{1}{2} \sum_{\mu} g_{i, i+\hat{y}, \mu}^{t}\left\langle c_{i \mu}^{\dagger} c_{i+\hat{y} \mu}\right\rangle+g_{i+\hat{y}, i, \mu}^{t}\left\langle c_{i+\hat{y} \mu}^{\dagger} c_{i \mu}\right\rangle, \\
K_{i} & =\left(K_{i, i+\hat{x}}+K_{i, i-\hat{x}}+K_{i, i+\hat{y}}+K_{i, i-\hat{y}}\right) / 4
\end{aligned}
$$

where $\Delta_{i}$ and $m_{i}$ are the pairing and spin order parameters on each site and $K$ is the bond order for determining the symmetry. The UPOP also plays an important role in our work. For those unidirectional patterns, we first calculate $\overline{\Delta_{x}}$ and $\overline{\Delta_{y}}$ :

$$
\begin{aligned}
& \overline{\Delta_{x}}=\sum_{\mathbf{K}} \sum_{i}^{N_{x}} \Delta_{i i+\hat{x}}^{\mathbf{K}} / N_{x} / M_{c} \\
& \overline{\Delta_{y}}=\sum_{\mathbf{K}} \sum_{i}^{N_{x}} \Delta_{i i+\hat{y}}^{\mathbf{K}} / N_{x} / M_{c}
\end{aligned}
$$

where $N_{x}$ is the lattice size in $\mathrm{x}$ direction and $M_{c}$ is the supercell size. $\mathbf{K}$ is the wave vector for different supercell and its form will be revealed later. $\hat{x}(\hat{y})$ is the unit vector in $\mathrm{x}(\mathrm{y})$ direction. After we obtain the averaged pairing values in $\mathrm{x}$ and $\mathrm{y}$ direction, we can then calculate UPOP:

$$
U P O P=\frac{\left|\overline{\Delta_{x}}\right|+\left|\overline{\Delta_{y}}\right|}{2}
$$

\subsection{Green's function and LDOS}

Since the patterns that we discuss their features of Green's function are all unidirectional, we can exploit the translational invariance in y-direction assuming that the modulation is in $\mathrm{x}$-direction to reduce the calculation time. By transforming our original cre- 
ation/annihilation operators into those with basis of $\left(i_{x}, k\right)$ :

$$
c_{i, \mu}^{\dagger}=\frac{1}{\sqrt{N}} \sum_{k} c_{i_{x}, \mu}^{\dagger}(k) e^{-i k R_{i_{y}}}
$$

we could translate our Hamiltonian as in a 1D lattice. With this transformation, we are able to perform the calculation for lattice size two times larger. For the symbols above, $N$ represents the lattice size in y-direction, $R_{i_{y}}$ is the y component of the original lattice vector $i$, and $c_{i_{x}, \mu}^{\dagger}(k)$ is the creation operator in this quasi-1D system for momentum $k$. Therefore the Hamiltonian becomes:

$$
\begin{aligned}
H= & \sum_{\left\langle i_{x}, j_{x}\right\rangle, k, \mu} H_{i_{x} j_{x} \mu}(k) c_{i_{x} \mu}^{\dagger}(k) c_{j_{x} \mu}(k)+h . c . \\
& +\sum_{\left\langle i_{x}, j_{x}\right\rangle, k, \mu} \sigma D_{i_{x} j_{x} \mu}^{*}(k) c_{i_{x} \mu}(k) c_{j_{x} \bar{\mu}}(-k)+h . c . \\
& -\sum_{i_{x}, k, \mu} \epsilon_{i_{x} \mu} n_{i_{x} \mu}(k)
\end{aligned}
$$

where

$$
H_{i_{x} j_{x} \mu}(k)=\sum_{i_{y}} H_{i_{x} i_{y} j_{x} 0 \mu} e^{-i k R_{i_{y}}}
$$

Similar expressions hold for $D_{i_{x} j_{x} \mu}(k)$ and $\epsilon_{i_{x} \mu}$. With converged values of the eigenfunctions, the Green's function matrix can be calculated using:

$$
\begin{aligned}
& G_{i j \mu}(\omega)=\frac{1}{N} \sum_{k} g_{i j \mu}^{t} G_{i_{x} i_{y} \mu}(k, \omega) e^{i k\left(R_{i_{y}}-R_{j_{y}}\right)} \\
& G_{i_{x} i_{y} \mu}(k, \omega)=\sum_{n>0}\left[\frac{u_{i_{x} \mu}^{n}(k) u_{j_{x} \mu}^{n *}(k)}{\omega-E_{n \mu}(k)+i 0^{+}}+\frac{v_{i_{x} \mu}^{n *}(k) v_{j_{x} \mu}^{n}(k)}{\omega+E_{n \bar{\mu}}(k)+i 0^{+}}\right]
\end{aligned}
$$

The broadening $0^{+}$can be several forms according to separate situation but is equal to $0.01 t$ if not specially mentioned. To compute the LDOS at the STM tip position, we 
change the basis and obtain the local continuum Green's function using [119].

$$
G_{\mu}(\mathbf{r}, \omega)=\sum_{i j} G_{i j \mu}(\omega) W_{i}(\mathbf{r}) W_{j}^{*}(\mathbf{r})
$$

where $W_{i}(\mathbf{r})$ is the Wannier function at site $\mathrm{i}$ and $\mathbf{r}$ is a three-dimensional continuum real space vector. The Wannier function employed was generated using Wannier90 package [120] and is similar in form to that used in [121]. Note that the local Green's function contains nonlocal contributions from all lattice sites. The continuum LDOS is now easily obtained as:

$$
\rho_{\mu}(\mathbf{r}, \omega)=-\frac{1}{\pi} \operatorname{Im}\left[G_{\mu}(\mathbf{r}, \omega)\right]
$$

In most of the previous theoretical works [ 51, 53, 32], intra-unit cell form factors were calculated using the Fourier transform of the nearest neighbor bond order $\chi_{i, i+\hat{x}(\hat{y})}$, which can be regarded as the measure of charge density at the oxygen atoms on $\mathrm{x}(\mathrm{y})$ bonds at lattice site $i$. We can express $s-, s^{\prime}-$, and $d$-form factors as follows.

$$
\begin{aligned}
& D(\mathbf{q})=F T\left(\widetilde{\chi}_{i, i+\hat{x}}-\widetilde{\chi}_{i, i+\hat{y}}\right) / 2 \\
& S^{\prime}(\mathbf{q})=F T\left(\widetilde{\chi}_{i, i+\hat{x}}+\widetilde{\chi}_{i, i+\hat{y}}\right) / 2 \\
& S(\mathbf{q})=F T\left(1-\widetilde{\delta}_{i}\right)
\end{aligned}
$$

where FT refers to the Fourier transform and $\sim$ denotes that the spatial average of the corresponding quantity has been subtracted to emphasize modulating components. Obviously, this quantity does not have any energy dependence. However, STM experiments utilized phase resolved sublattice LDOS information [28] to extract the form factors and found a significant bias dependence [1]. Using the continuum LDOS information, we can follow a similar approach. To analyze this behavior, we first we obtain LDOS $Z$-maps, defined below, on a plane located at a typical STMtip height $(\approx 5 \AA)$ above the BiO plane. 


$$
\rho^{Z}(\mathbf{r}, \omega>0)=\frac{\sum_{\mu} \rho_{\mu}(\mathbf{r}, \omega)}{\sum_{\mu} \rho_{\mu}(\mathbf{r},-\omega)}
$$

Next, we take non-overlapping square regions around each atom in the $Z$-map, with the size of the region identical to that used in the experiment [1], and subsequently assign it to the sublattice $Z$-maps $C u^{Z}(\mathbf{r}, \omega), O_{x}^{Z}(\mathbf{r}, \omega)$ and $O_{y}^{Z}(\mathbf{r}, \omega)$. We note that form factor results are not very sensitive to the size of the square region, however. Here subscripts $\mathrm{x}$ and y designate two nonequivalent oxygen atoms in the unit cell in horizontal and vertical directions, respectively. Taking the proper linear combination of the Fourier transform of the sublattice LDOS yields $s-, s^{\prime}-$, and $d$-form factors as follows:

$$
\begin{aligned}
& D^{Z}(\mathbf{q}, \omega)=\left(\widetilde{O}_{x}^{Z}(\mathbf{q}, \omega)-\widetilde{O}_{y}^{Z}(\mathbf{q}, \omega)\right) / 2 \\
& S^{\prime Z}(\mathbf{q}, \omega)=\left(\widetilde{O}_{x}^{Z}(\mathbf{q}, \omega)+\widetilde{O}_{y}^{Z}(\mathbf{q}, \omega)\right) / 2 \\
& S^{Z}(\mathbf{q}, \omega)=\widetilde{C u}_{x}^{Z}(\mathbf{q}, \omega)
\end{aligned}
$$

Another important quantity of interest is the average spatial phase difference $(\Delta \phi)$ between the positive and negative bias energies for the $d$-form factor modulations. To compute $\Delta \phi$ in accordance with the experimental procedure [80], we filter out the characteristic wave vector corresponding to $d$-form factor modulation $\left(Q_{d}\right)$ from the continuum LDOS maps at positive and negative energies using a Gaussian filter. Then we take the inverse Fourier transform to obtain the complex spatial map $D(\mathbf{r}, \omega)$ and determine its phase $\phi(\mathbf{r}, \omega)$. By taking the average of the spatial phase difference at $\pm \omega$, we find $\Delta \phi$ :

$$
\begin{aligned}
& D^{g}(\mathbf{q}, \omega)=\left(\widetilde{O}_{x}^{g}(\mathbf{q}, \omega)-\widetilde{O}_{y}^{g}(\mathbf{q}, \omega)\right) / 2 \\
& D(\mathbf{r}, \omega)=\frac{2}{(2 \pi)^{2}} \int d \mathbf{q} e^{i \mathbf{q r}} D^{g}(\mathbf{q}, \omega) e^{-\frac{\left(\mathbf{q}-\mathbf{Q}_{d}\right)^{2}}{2 \Lambda^{2}}} \\
& \phi(\mathbf{r}, \omega)=\tan ^{-1}(\operatorname{Im}[D(\mathbf{r}, \omega)] / \operatorname{Re}[D(\mathbf{r}, \omega)]) \\
& \Delta \phi=\langle\phi(\mathbf{r}, \omega)-\phi(\mathbf{r},-\omega)\rangle
\end{aligned}
$$

where $\widetilde{O}_{x}^{g}(\mathbf{q}, \omega)$ and $\widetilde{O}_{y}^{g}(\mathbf{q}, \omega)$ are the FT of the sublattice LDOS maps for oxygen $x$ and 
oxygen $y$. Width of the Guassian filter was taken to be $\Lambda=1 / 2 N$.

\subsection{Spectra weight and many-body Chern number}

Because we are going to investigate the features in $k$ space, it is necessary to apply the supercell calculation [122]. For each cell we have $N_{x} \times N_{y}$ sites and the total number of cell is $M_{c}=M_{x} \times M_{y}$. Our Hamiltonian is then reduced from $2 M_{x} N_{x} \times 2 M_{y} N_{y}$ to $M_{x} \times M_{y}$ matrix equation each with lattice size $2 N_{x} \times 2 N_{y}$. The self-consistent solutions now have to be carried out for each cell. The spectral weight can be written with our wave function $(u, v)$ as:

$$
\begin{aligned}
A(k, \omega)= & \frac{1}{N} \sum_{i j, n+} f\left(-E_{n}\right)\left(e^{i \mathbf{k} \cdot\left(\mathbf{r}_{i}-\mathbf{r}_{j}\right)} g_{i j \uparrow}^{t} u_{i, n}^{\mathbf{K} *} u_{j, n}^{\mathbf{K}} \delta\left(\omega-E_{n}\right)\right. \\
& \left.+e^{i \mathbf{k} \cdot\left(\mathbf{r}_{j}-\mathbf{r}_{i}\right)} g_{i j \downarrow}^{t} v_{i, n}^{\mathbf{K}} v_{j, n}^{\mathbf{K} *} \delta\left(\omega+E_{n}\right)\right) \\
& +\frac{1}{N} \sum_{i j, n-} f\left(E_{n}\right)\left(e^{i \mathbf{k} \cdot\left(\mathbf{r}_{i}-\mathbf{r}_{j}\right)} g_{i j \uparrow}^{t} u_{i, n}^{\mathbf{K} *} u_{j, n}^{\mathbf{K}} \delta\left(\omega-E_{n}\right)\right. \\
& \left.+e^{i \mathbf{k} \cdot\left(\mathbf{r}_{j}-\mathbf{r}_{i}\right)} g_{i j \downarrow}^{t} v_{i, n}^{\mathbf{K}} v_{j, n}^{\mathbf{K} *} \delta\left(\omega+E_{n}\right)\right)
\end{aligned}
$$

where $\mathbf{k}=\mathbf{k}_{0}+\mathbf{K}$ while $\mathbf{k}_{0}=2 \pi\left(\frac{n_{x}}{N_{x}}, \frac{n_{y}}{N_{y}}\right)$ where $n_{x} \in\left[-N_{x} / 2+1, N_{x} / 2\right], n_{y} \in\left[-N_{y} / 2+\right.$ $\left.1, N_{y} / 2\right]$, and $\mathbf{K}=2 \pi\left(\frac{n_{x}^{c}}{M_{x} N_{x}}, \frac{n_{y}^{c}}{M_{y} N_{y}}\right)$ where $n_{x}^{c} \in\left[0, M_{x}-1\right], n_{y}^{c} \in\left[0, N_{y}-1\right] . f\left(E_{n}\right)$ is the Fermi-Dirac distribution and $n+(n-)$ means summation over positive(negative) energies. $\delta\left(\omega-E_{n}\right)$ is the Lorenzian and has the following form:

$$
\delta\left(\omega-E_{n}\right)=\frac{1}{\pi} \frac{\Gamma}{\Gamma^{2}+\left(\omega-E_{n}\right)^{2}}
$$

Next, for each band flattened by magnetic field its Chern number is defined by inte- 
grating the Berry curvature over the first Brillouin zone [123]:

$$
\begin{aligned}
& C_{n}=\frac{1}{2 \pi} \sum_{\mathbf{k} \in B Z} \nabla_{\mathbf{k}} \times \vec{A}_{n}(\mathbf{k})=\frac{1}{2 \pi} \sum_{\mathbf{k} \in B Z} \vec{B}_{n}(\mathbf{k}) \\
& =\frac{-i}{2 \pi} \sum_{m \neq n} \sum_{\mathbf{k} \in B Z} \frac{\left\langle n \mathbf{k}\left|J_{x}\right| m \mathbf{k}\right\rangle\left\langle m \mathbf{k}\left|J_{y}\right| n \mathbf{k}\right\rangle-\left(J_{x} \leftrightarrow J_{y}\right)}{\left[E_{n}(\mathbf{k})-E_{m}(\mathbf{k})\right]^{2}}
\end{aligned}
$$

where $\vec{A}_{n}(\mathbf{k})=-i\left\langle n \mathbf{k}\left|\nabla_{\mathbf{k}}\right| n \mathbf{k}\right\rangle$ is the Berry vector field for the nth band, and $\vec{B}_{n}(\mathbf{k})$ is the related field. The current $\mathbf{J}=\left(J_{x}, J_{y}\right)$ is given by $J=\nabla_{\mathbf{k}} H$. 


\section{Chapter 3}

\section{Results I - High $T_{c}$ Cuprate}

\subsection{Real space properties}

The calculations of ours all start from a set of initial input of variational parameters of $\delta_{i}, \Delta_{i j \mu}^{v}, \chi_{i j \mu}^{v}$, and sometimes $m_{i}^{v}$. In most cases, we will obtain only uniform solutions such as the d-wave superconducting(dSC) state and/or coexistent antiferromagnetic(dSCAFM) state, but sometimes the states with ordered patterns are found as a self-consistent solution.

\subsubsection{Charge-ordered patterns}

In addition to the two uniform solutions of a dSC state and a dSC-AFM state, there are many non-uniform charge-ordered states. For simplicity, we shall first present those charge-ordered states with a period of four lattice spaces $\left(4 a_{0}\right)$, as listed in Table 3.1. Both the pair field $\Delta_{i j \mu}^{v}$ and the spin moment $m_{i}^{v}$ could have positive and negative values. It turns out that if there is a SDW or a bidirectional spin $\mathrm{CB}(\mathrm{sCB})$ present, then it always has a period of $8 a_{0}$, with two domains of size $4 a_{0}$ with opposite antiferromagnetic directions joining together. The pair field has more choices. It could always be positive, with all of its $\mathrm{x}$-bond pair field being positive and $\mathrm{y}$-bond pair field being negative: thus, it would have a net total non-zero pair field. This is called an in-phase(IP) state, with a period of $4 a_{0}$. However, just like the spin moment, the pair field could also have two domains with 

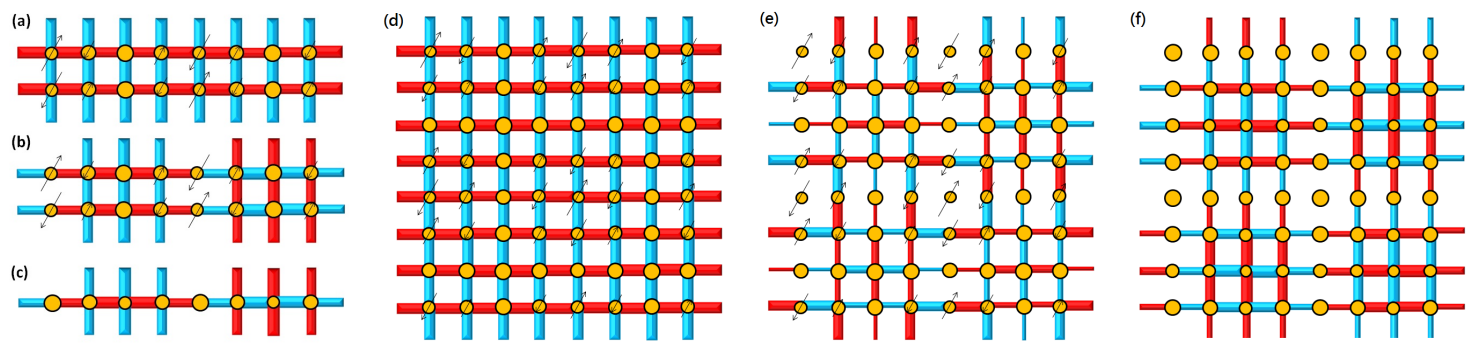

Figure 3.1: Schematic illustration of modulations for stripe like patterns. (a) IP-CDWSDW (b) AP-CDWSDW (c) AP-CDW (d) IP-cCB-sCB (e) AP-cCB-sCB (f) AP-cCB respectively. Size of the circle represents the hole density. The width of the bond around each site represents the amplitude of pairing $\Delta\left(\Delta=\sum_{\mu} \Delta_{\mu}\right)$ and sign is positive (negative) for red (cyan). The size of black arrows represents the spin moment. The average hole density is about 0.1 but 0.09 for IP-cCB-sCB.

opposite signs and a domain wall in between: this state is known as the anti-phase(AP) state, with a period of $8 a_{0}$. Thus, we could have four possible states for each unidirectional CDW or bidirectional charge $\mathrm{CB}(\mathrm{cCB})$, as we either have an IP or AP pair field with or without SDW. However, we only have three such states in Table 3.1 because we cannot find a solution with an IP pair field and CDW both in $4 a_{0}$ period. Later, we will show a state with a net pairing order or IP pairing state and CDW, which occurs if we do not require solutions to be commensurate with the lattice and that is the nPDW state.

\begin{tabular}{|l|l|l|l|}
\hline & pair field & charge modulation & spin modulation \\
\hline IP-CDW-SDW & in-phase & stripe & yes \\
\hline AP-CDW-SDW & anti-phase & stripe & yes \\
\hline AP-CDW & anti-phase & stripe & zero \\
\hline IP-cCB-sCB & in-phase & checkerboard & yes \\
\hline AP-cCB-sCB & anti-phase & checkerboard & yes \\
\hline AP-cCB & anti-phase & checkerboard & zero \\
\hline dSC & uniform & uniform & zero \\
\hline dSC-AFM & uniform & uniform & uniform \\
\hline diag & in-phase & stripe along $(1,1)$ & yes \\
\hline
\end{tabular}

Table 3.1: Definition of various nearly degenerate states with respect to the intertwined orders: pair field, charge density, and spin moment. Besides the two uniform solutions, dwave superconducting(dSC) state and coexistent antiferromagnetic(dSC-AFM) state, all the states to be considered in this paper, unless specifically mentioned, have modulation period $4 a_{0}$ for charge density and bond order. IP(AP) means the pair field is in-phase with period $4 a_{0}$ (anti-phase with period $8 a_{0}$ ). IP has a net pairing order and AP has none. SDW is the spin density wave with period $8 a_{0} . \mathrm{sCB}(\mathrm{cCB})$ denotes the checkerboard pattern of spin(charge) and diag means the diagonal stripe which has in-phase pair field and spin modulation. 
(a)

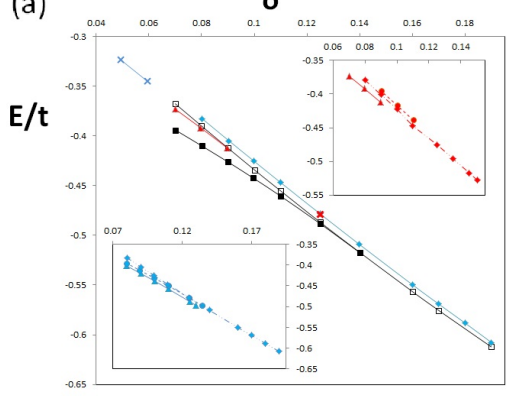

(b)

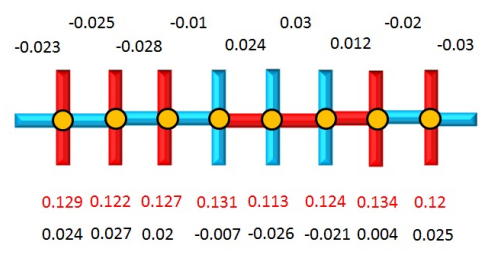

(c)

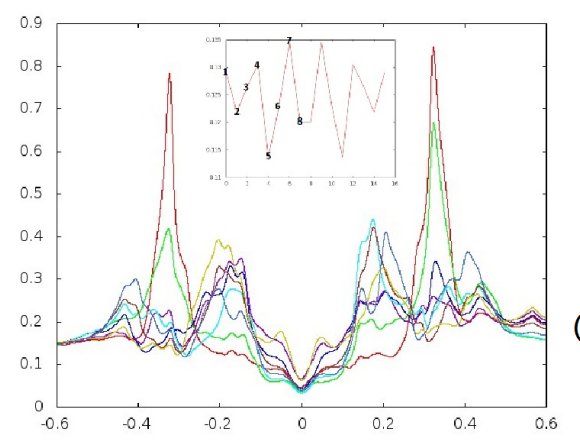

- Site 1

- Site 2

- Site 3

- Site 4

- Site 5

- Site 6

- Site 7

- Site 8

(d)

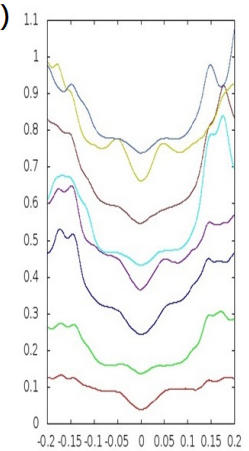

Figure 3.2: (a) Energy per site as a function of hole concentration. Six states are shown in the main figure with notations defined in Table 2. The lower (upper) inset is for stripe (CB) patterns. Blue triangles, circles, and diamonds are for IP-CDW-SDW, AP-CDW-SDW, and AP-CDW respectively. And red triangles, circles and diamonds are for IP-cCB-sCB, AP-cCB-sCB, and AP-cCB respectively. (b) Schematic illustration of modulations for nPDW stripe. The numbers in red denote the hole density at each site while the numbers in black below them represent the pairing amplitude in y direction. The rest numbers above the figure stand for the pairing amplitude in $\mathrm{x}$ direction. Here our pairing amplitudes denote $\left(\left\langle c_{i \uparrow} c_{j \downarrow}\right\rangle\right)$. Note that in this figure neither the size of circles nor the width of bonds represent amplitudes. The hole concentration is 0.125 . (c) LDOS at 8 sites plotted from energy $0.6 \mathrm{t}$ to $-0.6 \mathrm{t}$. The inset shows hole density along the modulation direction of the nPDW stripe and (d) from $0.2 t$ to $-0.2 t$ but shifted vertically for clarity.

Figure 3.1 shows a schematic illustration of the modulations of the pair field, charge density and spin moment for the three stripes and three checkerboards with hole concentration of 0.1 or 0.09 . The magnitude of the pair field is proportional to the width of the bond; red (cyan) denotes positive (negative) value. The size of the arrow is proportional to the spin moment, and the size of the circle represents the hole density. There is one domain wall corresponding to the vanishing spin moment in Fig. 1(a) and (d) or the vanishing pair field in Fig. 1(c) and (f). Both domain walls are present in Fig. 1(b) and (e). The hole density is always maximum at the domain wall with the vanishing spin moment. However, if there is no SDW, such as the AP-CDW stripe in Fig. 1(c), then the hole density is maximum at the domain wall with the vanishing pair field. This finding is different from previous work without including the renormalized chemical potential [48]. 
Figure 3.2(a) shows energies as a function of hole concentration for all the states listed in Table 3.1. The three unidirectional states are shown in the lower inset with blue triangles, circles, and diamonds representing IP-CDW-SDW, AP-CDW-SDW, and AP-CDW, respectively. The three $\mathrm{CB}$ states are shown in the upper inset with red triangles, circles and diamonds representing IP-cCB-sCB, AP-cCB-sCB, and AP-cCB, respectively. Those patterns are all site-centered here but bond-centered solutions have essentially the same energies. The same results for the three CDW states were also reported in ref. [40] at a 1/8 hole concentration. These mean-field GWA results are quite consistent with the numerical Monte Carlo result [43], which revealed that the uniform state has the lowest energy, followed by the in-phase stripe, and that the energy of the anti-phase stripe is slightly above that of both of them. However, the small energy differences are insignificant compared to the result of iPEPS [54], which showed the same ordering of states but with essentially degenerate energies.

At approximately 12\% doping in Fig. 3.2(a), the spin moment becomes smaller, and the uniform dSC-AFM state merges into the dSC state. The difference from the original work of Ogata and Himeda [44, 45], in which the spin moment vanished at 10\% doping, is due to the simplified Gutzwiller factors used in Eq. 6.5. All the magnetic states, such as SDW and sCB, vanish at approximately $12 \%$ doping. The most surprising and important result shown in Fig. 3.2(a) is that in addition to the uniform dSC state, the AP-CDW state is most stable for a large doping range, from 0.08 to 0.18 . The AP-cCB state also extends a little bit beyond the antiferromagnetic region. We only find the diagonal stripe state up to $6 \%$ doping. Another pattern that seems to be limited to small doping is IP-cCB-sCB, which is only found at doping less than 0.1 . The general locations of these $\mathrm{CB}$ states in Fig. 3.2(a) are consistent with experimental observations that $\mathrm{CB}$ are seen more often at low doping [24, 25]. Because the Gutzwiller factor $g_{i j \mu}^{t}$ in Eq. 6.5 is proportional to the hole density at the site, we expect the kinetic energy to be maximum at the domain wall (Fig. 3.1(c),(f)). The hopping and pairing order are calculated from respectively, by using Eq. 6.14.

The red cross in Fig. 3.2 (a) at the 1/8 hole concentration is the energy of nPDW that 
relaxes the requirement to have a commensurate $4 a_{0}$ period for the AP-CDW state. To alleviate the difficulty of considering incommensurate solutions in a finite lattice calculation, we allow the state to have more than one single modulation period. In Fig. 3.2(b), the hole density, listed as the red numbers below the pattern, along with the magnitude of the pairing order parameter for both $\mathrm{x}$ and $\mathrm{y}$ bonds, listed in the top and bottom rows, are plotted along the direction of the modulation. It is very similar to the AP-CDW state. However, there is a remaining net constant d-wave pairing, with the system average $\Delta_{x}=-0.0056$ and $\Delta_{y}=0.0057$. This mixture of the AP-CDW stripe with a small constant uniform pairing will produce a $d$-wave nodal-like LDOS in addition to a PDW; hence, we name it nodal PDW or nPDW. There are several important results associated with the nPDW. Figure 3.2(b) shows that the hole density is indeed maximum at the domain walls near sites $4,7,10$ and 13 . The maximum amplitude of pairing order $\Delta$ is about 0.03 , which is roughly the same as adding the net pairing amplitude to that of the AP-CDW stripe. It is most gratifying to observe that the $d$-wave pairing is globally maintained, although we have no way of controlling it during the iteration, with variables changing independently on each site. Contrary to the pure AP-CDW state without a net pairing, this state has a $d$-wave nodal spectrum at low energy, hence a nodal-like LDOS. In Fig. 3.2(c), the LDOS of this stripe at 8 sites is plotted as a function of energy. The positions of these 8 sites are indicated in the inset of Fig. 3.2(c). The detailed LDOS at low energy is shown in Fig. 3.2(d). The large spatial variation of LDOS at high energies but always with a $d$-wave node near zero energy is quite consistent with the STM results in ref. [15]. We have obtained this result by using a lattice of supercell calculation.

A special feature of all these charge-ordered states is the large variation of the Gutzwiller factors from site to site. The values could change between nearest neighbors by a factor of 2 to 3 . This unique property of strong correlated systems originates from the dependence on local hole density in the Gutzwiller factor, which is $g_{i}^{t}=\sqrt{\frac{2 \delta_{i}}{1+\delta_{i}}}$, when we do not consider magnetic moments. This dependence on $\delta_{i}$ is the consequence of being a Mott insulator when there are no doped holes. A slight variation of the hole density $\delta_{i}$ will cause a large change in $g_{i}^{t}$; in fact, $\partial g_{i}^{t} / \partial \delta_{i}$ is proportional to $g_{i}^{t} / \delta_{i} \sim 1 / \sqrt{\delta_{i}}$. This factor 


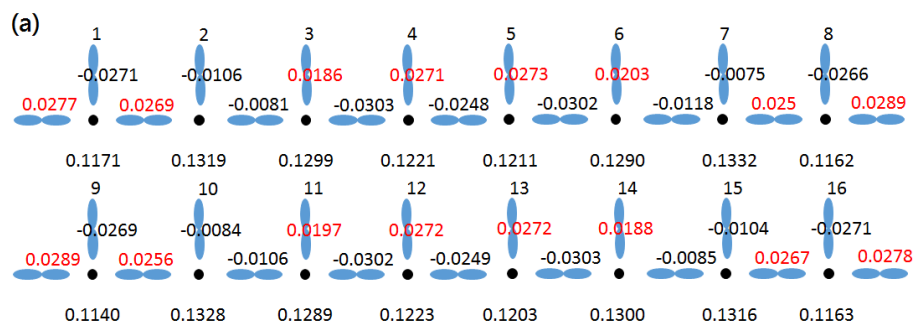

(d)
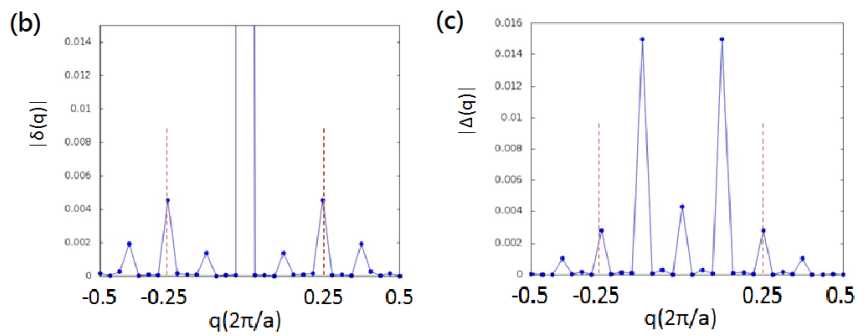

(e)
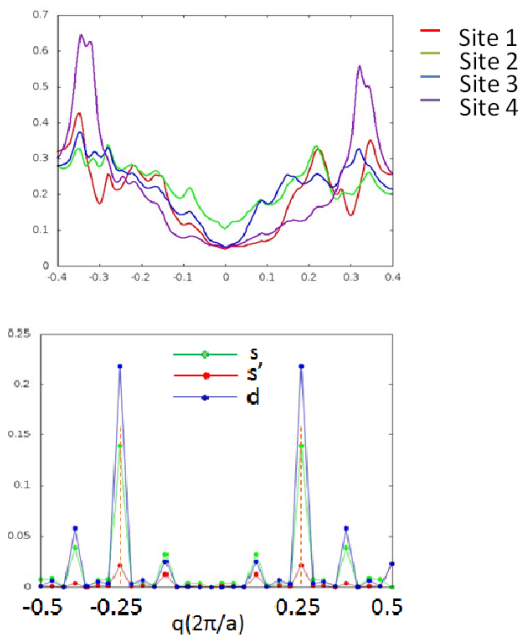

Figure 3.3: Properties of nPDW. (a) The real space modulation of nPDW in $32 \times 32$ lattice sites with $\delta=0.125$. Since the pattern repeats itself with an inversion symmetry in the middle bond, here we only show the first 16 sites. The red and black numbers on each bond denote the values of pairing order and the number at each site(black dots) is the hole density. (b)(c) The Fourier transform of the value of hole density(b) and pairing order(c). (d) LDOS of the first 4 sites of this $32 \times 32 \mathrm{nPDW}$. (e) Different form factors.

dominates in the renormalized local chemical potential defined in Eq. 6.10 when hole concentration is small. Thus, $g_{i}^{t}$ is no longer a purely passive renormalization factor; now, it could alter the local chemical potential greatly and induce non-uniform charge orders. Although the factor associated with spin, $g_{i}^{s, x y}$ in Eq. 6.5, is smaller, it also contributes to the local chemical potential. The strong susceptibility to the variation of local hole density makes a uniform state unstable amidst inherent or extrinsic charge fluctuations. This effect is clearly more prominent in the lightly hole-doped regime, as demonstrated by the greater variety of charge-ordered states in the underdoped regime in Fig. 3.2(a). Another important effect of the Gutzwiller factor is that it introduces nonlinearity into the Bogoliubov-deGenne (BdG) equations (Eq. 6.9-6.10), which can produce quite unexpected solutions.

The nPDW state first proposed by us comes from the AP-CDW state but with a nonzero uniform pairing order UPOP, which is generated from its (quasi-)incommesurate nature. This accords with previous experimental data [1] that within the superconducting dome, the modulations of $\mathrm{Cu}-\mathrm{O}$ surface observed are incommensurate. Fig. 3.3 shows some basic characteristics of nPDW in a $32 \times 32$ lattice size. Fig. 3.3 (a) shows that the 
(a)

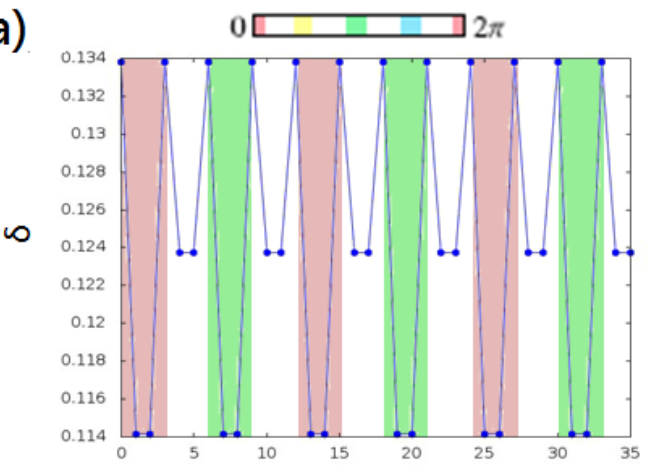

(c)

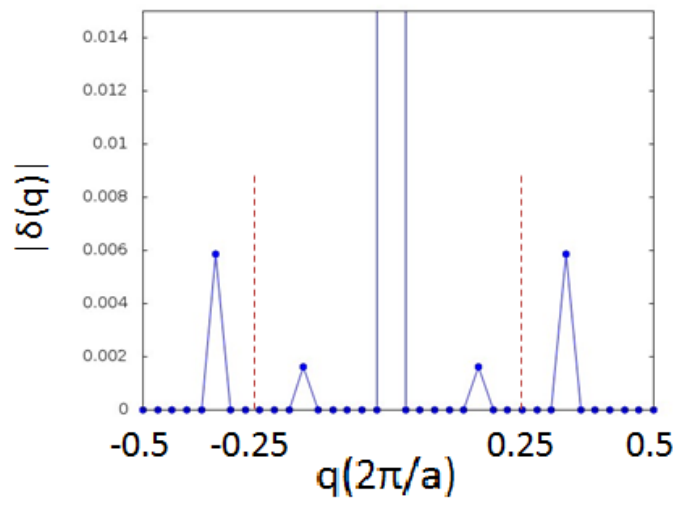

(b)

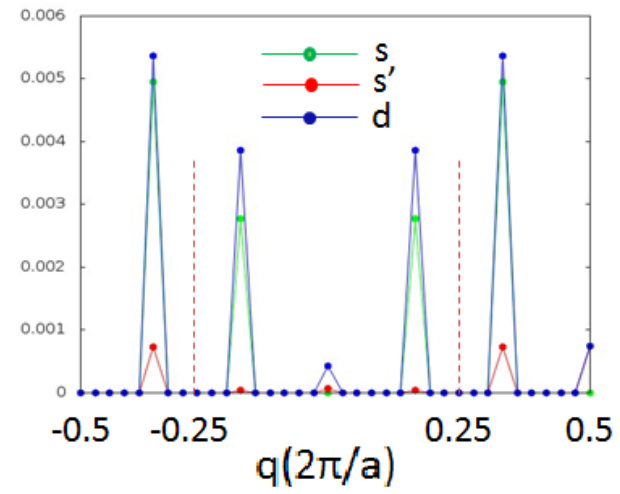

(d)

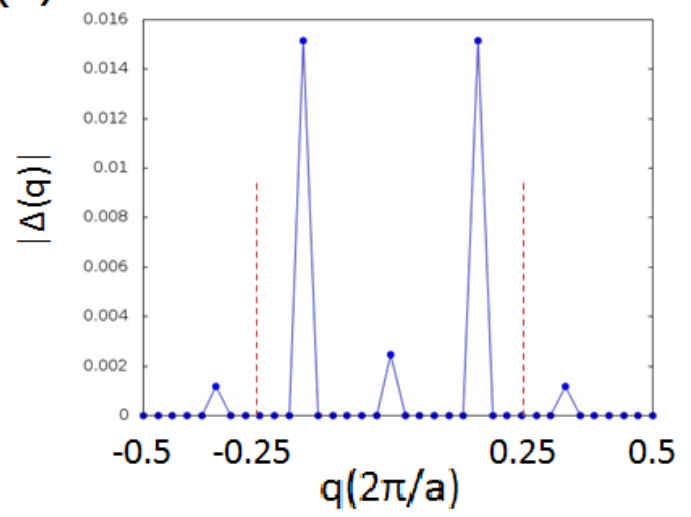

Figure 3.4: Figures showing the properties of discommensurate nPDW. (a) The phase variation of this pattern. Site $0-3,12-15$, and $24-27$ are of phase equal to $0(2 \pi)$ while sites 6-9, 18-21, and 30-33 are of phase $\pi$. (b) Form factors for discommensurate nPDW. We also include the Fourier transform of hole density(c) and pairing order(d).

hole density is maximum at the domain walls near sites 2,7,10 and 15 . For the Fourier transform in Fig. 3.3(b) and 3.3(c), it is clear that although there are several peaks, the leading one is the one at $\pi / 2(\pi / 4)$ for hole density(pairing order), which corresponds to the modulation of $4 a_{0}\left(8 a_{0}\right)$. Fig. 3.3(d) demonstrates LDOS of several chosen sites and the $v$-shape near zero energy indicates a $d$-wave pairing gap with a node is opened. Finally, in Fig. 3.3(e) the comparison of different form factors confirms the dominance of $d$-form factor.

Besides the (quasi-)incommensuration, McMillan [124] was the first to define a "discommensuration"(DC) as a defect in a commensurate CDW state. In such state, the phase of the CDW jumps between discrete lattice-locked values. Mesaros et al. [125] showed that this kind of CDW could be what was observed by experiments. Hence, let us consider a sinusoidal modulation in one spatial dimension with $4 a_{0}$ modulation but a phase jump 

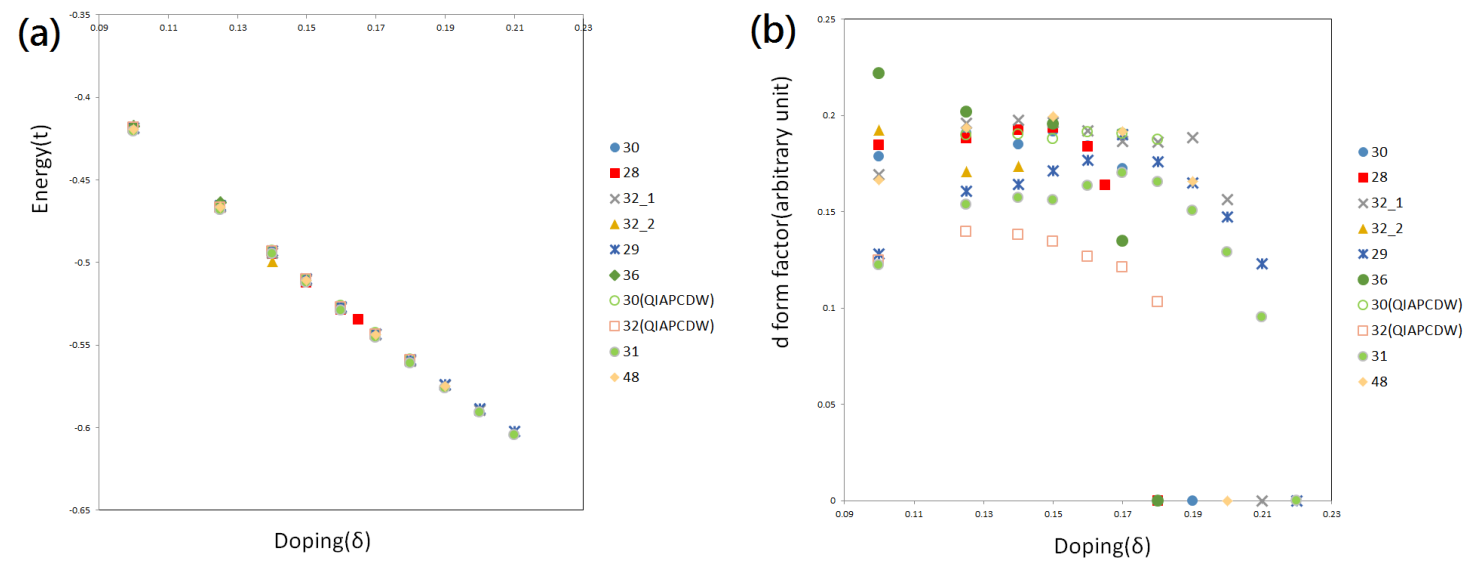

Figure 3.5: (a) Energies of several states chosen by us. Although we have listed ten different states here, their energies seem to be nearly degenerate and follow the same trend line. (b) Magnitude of $d$ form factor of patterns. Given different states we expect their magnitude to change but still all of them seem to have the same trend: the magnitude maintains the same until doping level exceeds 0.18 , where it starts to drop drastically and becomes zero in the range of $0.18 \sim 0.22$.

between each domain, it can be written as:

$$
\psi(x)=A \exp \left[i\left(Q_{0} x+\phi\right)\right]
$$

where $A$ is the amplitude and $Q_{0}=4 a_{0}$. The additional phase $\phi$ defines the phase shift for each domain. For example, Fig. 3.4(a) shows the modulation in x-direction for one of the DC patterns we have obtained. We will name it after the discommensurate nPDW state(DCnPDW). It is clear that there are two separate domains, one with pink color(sites $0-3,12-15$, and 24-27) with $\phi=0$ and the other with Green color(sites 6-9, 18-21, and 30-33) with $\phi=\pi$. Moreover, in Fig. 3.4(b)-(d) we can see its FT shows that the averaged modulation is no longer $4 a_{0}$. This might explain for the reason why there are some experiments which ended up observing the modulation period of $4 a_{0}$ but the others with incommensurability. They can originate from the same phase with local $4 a_{0}$ feature but a global incommensurability. Even with the discommensurability, however, they still possess the same dominant symmetry. As shown in Fig. 3.4(b), the leading form factor is still $d$-form.

One of the most important points we need to clarify is that despite the nPDW and DCnPDW already mentioned, we can easily obtain a number of different states by changing the 
initial inputs or lattice size. Each of them has slightly different values of pairing, charge density and bond orders. Fig. 3.5 (a) lists some of the examples and demonstrates their energies. One can see that in fact their energies are nearly degenerate, although the lattice size is different. Even with the same lattice size, it is also possible to have two distinct patterns, as shown in Fig. 3.5 (a). Within the states shown, there are two of them labeled with QI-APCDW, which is the abbreviation of quasi-incommensurate anti-phase CDW. Different from nPDW, this pattern has zero UPOP, just like AP-CDW. Amazingly, even though QI-APCDW and nPDW seem very different because of the existence of UPOP, these two still share nearly degenerate energies. Based on this discovery, we claimed that in fact all the orders $(\Delta, \chi$, etc.) are, instead of competing, intertwined and influencing each other. That is why such different states can possess nearly the same energy. We have to make it clear that the patterns listed here are only some of the possibilities and in fact there can be many more different states. Moreover, we like to point out that these QI-APCDW states are quite similar with the IPDW states, except the latter is generated by raising the temperature of the nPDW states, which we will discuss in detail later. Except for their energies, there are also some characteristics which these states all share with. One of them is the $d$ form factor symmetry. Among all these states, surprisingly, they all have leading $d$-form factor over $s$ and $s^{\prime}$, which is one of the important feature of nPDW. In Fig. 3.5(b), we have collected the values of magnitude of $d$-form factor for each states. We can see although for different states their magnitudes of $d$-form factor vary from each other, most values are within the range from 0.15 to 0.2 . Moreover, the ending points are all within the range of $0.18 \sim 0.22$, which is very near the quantum critical point observed by experiment around 0.19 .

\subsubsection{Continuum LDOS}

For a more fruitful comparison with STM experiment, we turn to the continuum LDOS and quantities derived from it. With the first-principles Wannier function for BSCCO2212 [121] as an input, we compute the continuum LDOS using equations 6.21 and 6.22. The resulting LDOS map at energy $\omega=0.25 t$ and in a $20 \times 20$ unit cell area at a height 
(a)

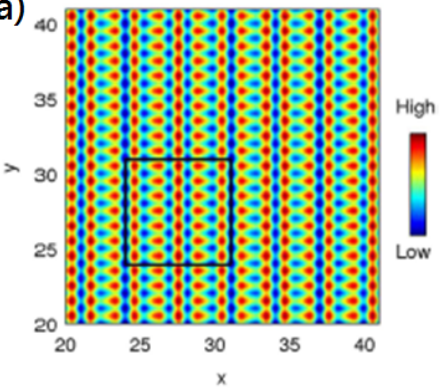

(b)

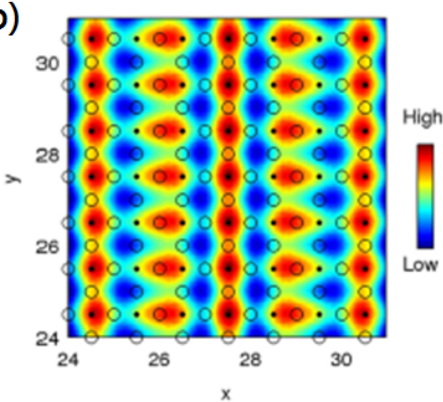

(c)

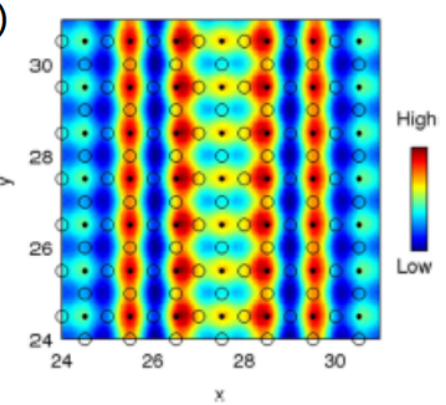

Figure 3.6: Continuum LDOS map at $\omega= \pm 0.25 t$ and $\sim 5$ angstrom above BiO plane. (a) LDOS map at $\omega=0.25 t$ in a range of $20 \times 20$ unit cells located in the central region of $60 \times 60$ lattice. (b) Zoomed-in view of the area marked by square in (a). Black dots and open circles represent positions of $\mathrm{Cu}$ and $\mathrm{O}$ atoms, respectively, in the $\mathrm{CuO}$ plane underneath. (c) LDOS map at $\omega=-0.25 t$ in the same region as in (b).

$z \sim 5$ angstrom above $\mathrm{BiO}$ plane, which is a typical height for STM tip, is shown in figure 3.6(a). Two types of modulating stripe structures can be observed. In figure 3.6(b) we plot a zoomed-in view of one of these structures in the area bounded by a square as shown in 3.6(a). $\mathrm{Cu}$ and $\mathrm{O}$ atoms located in the $\mathrm{CuO}$ plane underneath, are represented as dots and open circles, respectively. The LDOS shows modulations around all atoms which, in the Fourier domain, implies that this particular bias has a mixture of all intra-unit cell form factors.

More importantly, modulations at the two inequivalent $\mathrm{O}$ atoms in an unit cell $\left(O_{x}\right.$ and $O_{y}$ ) are out of phase, i.e. when $O_{x}$ has large LDOS then $O_{y}$ has small LDOS. This leads to the conclusion that the $d$-form factor has larger weight than $s^{\prime}$-form factor. Indeed, a more quantitative analysis of form factors, discussed in following paragraphs, shows that the $d$-form factor has largest weight at this particular bias. This particular pattern is observed in an energy range of $0.21 t-0.27 t$. Remarkably, a similar pattern has been observed in the STM experiments [1, 28]. In figure 3.6(c) LDOS map is plotted at the negative bias $\omega=-0.25 t$ in the same region as in (b). Comparing figures $3.6(b)$ and (c), it is found that the atoms with larger values of LDOS at positive bias have smaller values at negative bias, which implies a spatial phase change of $\pi$ between positive and negative biases. As emphasized in [1], this is a characteristic feature of $d$-form factor density wave. A more quantitative analysis of the phase differences, calculated using equation 6.26 , is given in following paragraphs. 

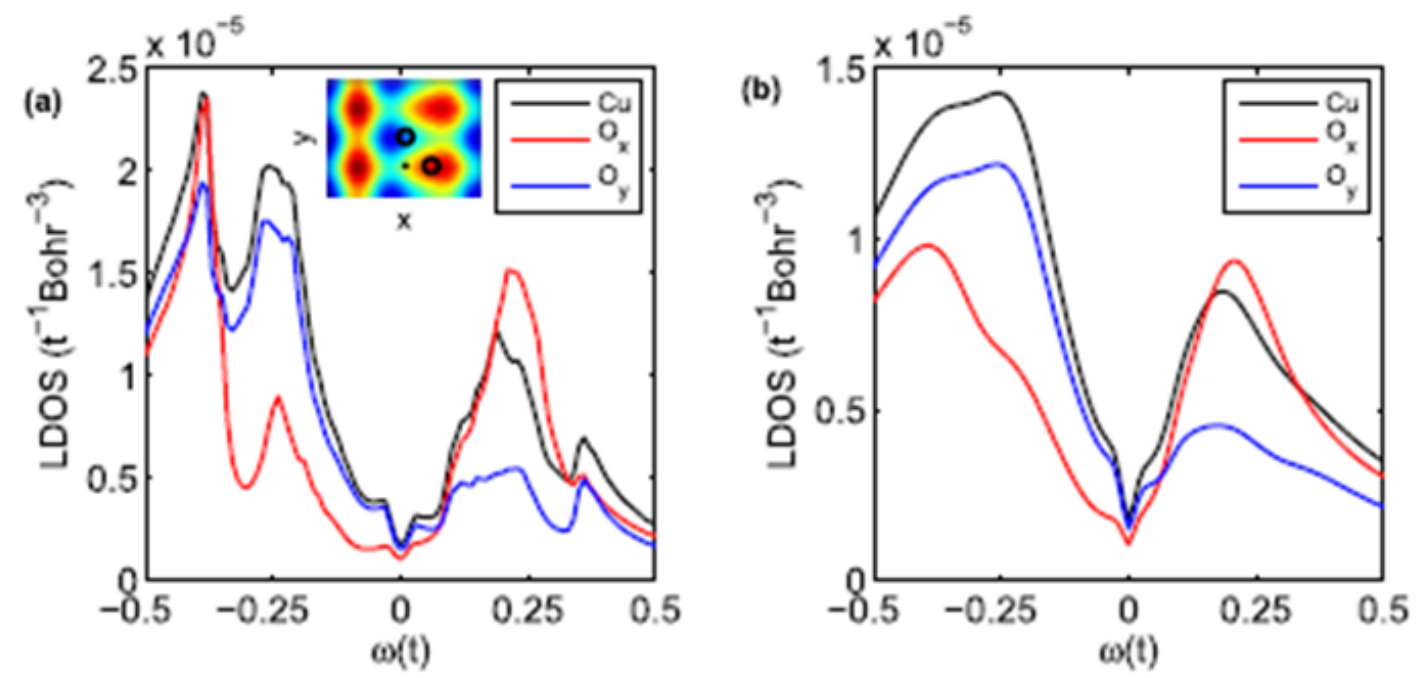

Figure 3.7: Continuum LDOS spectrum registered above $\mathrm{Cu}, O_{x}$ and $O_{y}$ sites in the unit cell $(25,25)$ at an height $\sim 5$ angstrom above $\mathrm{BiO}$ plane (a)without, and (b)with $\Gamma=\alpha|\omega|$ inelastic scattering $(\alpha=0.25)$, as extracted in [2]. The location of the unit cell can be referred from figure 4(b) as shown in the inset. Dots and open circles represent $\mathrm{Cu}$ and $\mathrm{O}$ atoms, respectively.

\subsubsection{Bias and doping dependence}

Figure 3.7 (a) shows the bias dependence of continuum LDOS at $\mathrm{Cu}, \mathrm{Ox}$, and $\mathrm{Oy}$ atomic positions in unit cell $(25,25)$ of a $60 \times 60$ system located at a height $\sim 5$ angstrom above the surface $\mathrm{BiO}$ plane. The location of this particular unit cell in reference to others can be found in the lower left corner of figure 3.6(b), and the cell is shown explicitly in the inset of figure 3.7(a). Similar to the lattice LDOS, two sets of "coherence peaks" at $\sim \pm 0.21 t$ and $\pm 0.37 t$ can be observed. These peaks correspond to themodulated Andreev state created by the PDW and that associated with the charge density wave energy scale [40].

Also, a small v-shaped gap-like feature exists around the Fermi level due to the uniform component of the gap order parameter. The most striking feature is the difference between the LDOS at $O_{x}$ and $O_{y}$ atoms, which clearly demonstrates intra-unit cell $C_{4}$ symmetry breaking. The difference between the two is maximum at $w \sim \pm 0.21 t$, the scale corresponding to the hybridized Andreev bound state(ABS). As we will see, this is the bias at which $d$-form factor has largest magnitude. Another feature of the LDOS is the strong particle-hole asymmetry. Interestingly, this asymmetry is seen to be much more pronounced in the continuum LDOS than lattice LDOS (figure 3.2(c)). 

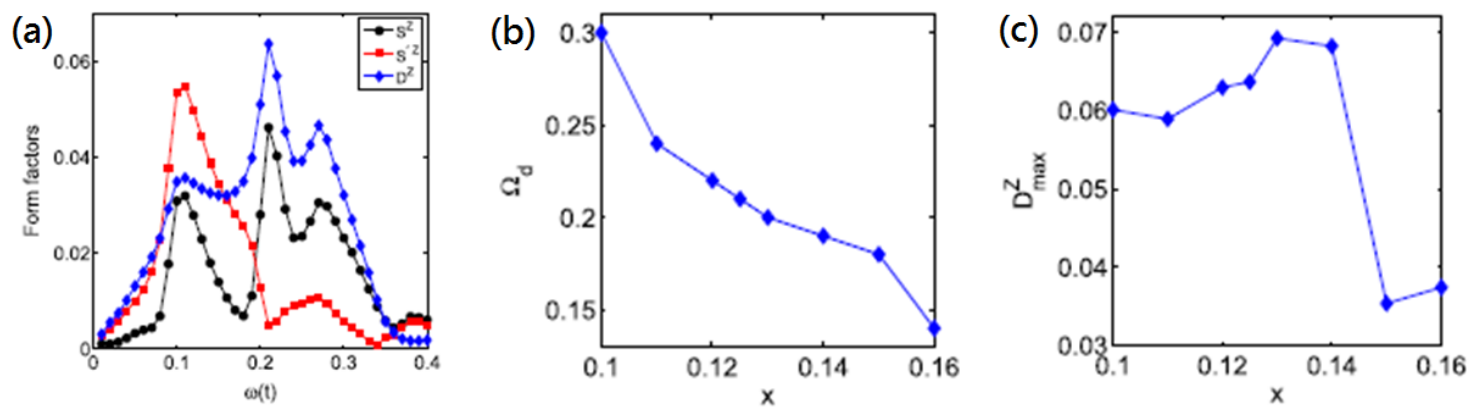

Figure 3.8: (a) Bias dependence of the intra-unit cell form factors at $x=0.125$ computed from atomic sublattice averages as described in the text.Next to it are the doping dependence of (a) energy at which d-form factor peaks $\left(\Omega_{d}\right)$ and (b) corresponding magnitude $\left(D_{\max }^{Z}\right)$.

We expect that these intra-unit cell contrast of these various effects will be mitigated somewhat when nonzero tip size is accounted for [121]. In addition, we expect the higherenergy features to be broadened significantly by inelastic scattering [2]. To see this effect explicitly, we incorporate linear inelastic scattering by replacing the constant artificial broadening term $(i 0+)$ in equation 6.20 by an energy dependent artificial broadening $i 0+i \Gamma(\omega)$ where $\Gamma(\omega)=\alpha|\omega|$, as observed in [2]. Figure 3.7 (b) shows the resulting continuum LDOS spectrum for $\alpha=0.25$. We find that high energy peaks are indeed broadened and can not be resolved any more. This holds for all higher values of $\alpha$. The spectrum resembles those taken on $O_{x}, O_{y}$, and $\mathrm{Cu}$ sites very closely [15]. We note that the value of spectral gap in the BSCCO-2212 spectrum reported in [15] is in the range $80-90 \mathrm{meV}$ for which the value of $\alpha$ is found to be in the range $0.25-0.33$, justifying our choice [2].

Using the continuum LDOS map, we can calculate energy dependent form factors as formulated in equation 6.25 . To calculate the wave vector corresponding to $d$-form factor modulation $\left(Q_{d}\right)$,we compute the $d$-form factor $\left(D^{Z}(\mathbf{q}, \omega)\right)$ as a function of energy and obtain the wave vector at which it peaks. We find that above a threshold bias, this wave vector does not show any dispersion and remains constant at $Q_{d}=(0.3,0)$. This non dispersing behavior is very similar to that seen in the experiment [1].

The energy dependence of the form factors at wave vector $Q_{d}=(0.3,0)$ is now shown in figure 3.8 (a). Similar to the experiment,we find an $s^{\prime}$-form factor peak at a lower energy and a $d$-form factor peak at higher energy. Comparing the energy scales in the lattice 

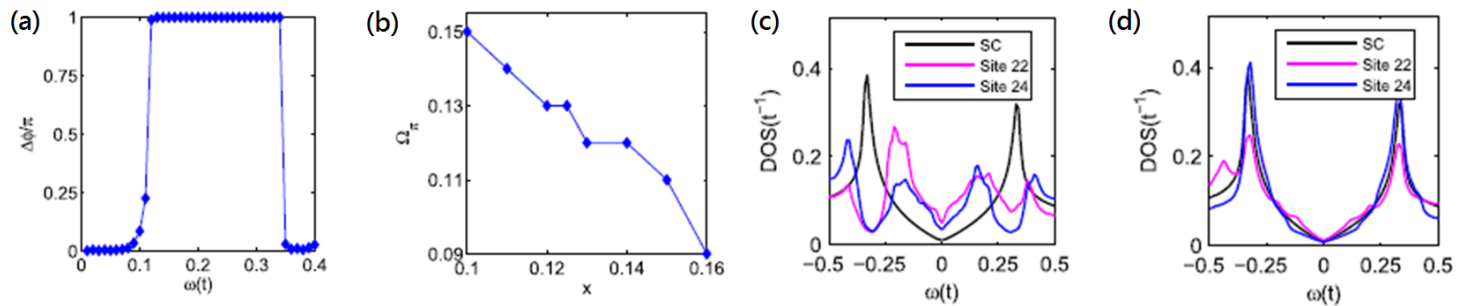

Figure 3.9: (a) Bias dependence of average spatial phase difference defined in equation (18). (b) Bias $\Omega_{p}$ at which initial $\pi$ phase jump in $\Delta_{\pi}$ takes place versus doping. (c) Lattice LDOS in the case when nPDW charge and bond modulations are turned off keeping only pair field modulations. (d) Lattice LDOS in the case when nPDW pair field modulations are turned off keeping charge and bond modulations.

LDOS (figure 3.2(c)) and continuum LDOS (figure 3.7), we find that the energy at which $d$-form factor peaks $\left(\Omega_{d}\right)$, corresponds to the ABS peak. By studying the bias dependence of form factors in systems with varying $t^{\prime}$, doping level and modulation wave vectors, we find that the $d$-form factor always displays a peak and the particular bias at which it occurs corresponds to the ABS peak in the lattice LDOS. However, the relative weight of the $s^{\prime}$ - and $d$-form factor depends on the details of band structure and doping. For example if we choose $t^{\prime}=0$ at the hole doping 0.125 , then $d$-form factor is found to have largest magnitude at all energies. Lastly, we note that the magnitude of the $s$-form factor is comparable to others(although it is never the strongest channel), whereas experiment finds it to be smaller than the others.

The doping dependence of the peak value of the $d$-form factor $\left(D_{\max }^{Z}\right)$ and corresponding bias $\left(\Omega_{d}\right)$ is shown in figure 3.8 (b) and (c). $\Omega_{d}$ decreases monotonically with hole doping. On the other hand, $D_{\max }^{Z}$ shows a non-monotonic behavior as function of hole doping. First, it increases and achieves a maximum at doping $x=0.13$ and then drops rapidly. This is in agreement with the doping dependence of the STM intensity at the density wave modulation wave vector which can be thought as a measure of $d$-form factor magnitude [29].

The average spatial phase difference $(\Delta \phi)$ between the $d$-form factor density wave modulations at positive and negative biases, computed using equation 6.26, is shown in figure 3.9 (a).We find at $x=0.125$ that in the vicinity of Fermi level spatial phase difference is zero and turns to $\pi$ for $\omega>0.12 t$. This bias dependence is in excellent agreement 

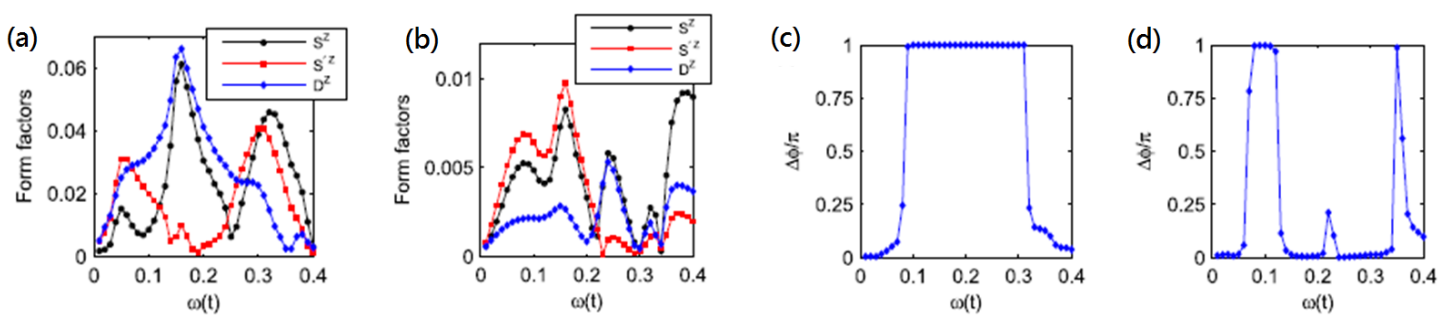

Figure 3.10: (a) and (c) Form factors and average spatial phase difference $(\Delta \phi)$ in the case when nPDW charge and bond modulations are turned off keeping only pair field modulations. (b) and (d) Form factors and average spatial phase difference $(\Delta \phi)$, respectively, in the case when nPDW pair field modulations are turned off keeping charge and bond modulations.

with the STM experiment [1]. Figure 3.9(b) shows that the energy $\left(\Omega_{\pi}\right)$ at which $\pi$ phase shift occur decreases with hole doping. We note that in the supplementary information of [1], the authors show the bias dependence of $\Delta \phi$ at a few more doping levels, from which one can infer that the energy corresponding to $\pi$ phase shift decreases with increasing hole doping level, similar to what we observe for the nPDW state.

To get a better understanding of the bias dependence of form factors and spatial phase difference, we attempt to disentangle PDW and CDW orders intertwined in the nPDW state, "by hand". We start with the self-consistent mean fields in the nPDW state discussed previously. As a first test, we do the following replacements in equation 6.11: $\delta_{i} \rightarrow$ $\delta_{0}$ and $\chi_{i j \mu}^{v} \rightarrow \chi_{0}^{v}$, where, subscript 0 indicates that the mean fields correspond to the uniform superconducting state. The pair field remains inhomogeneous and unchanged from the nPDW solution. In the second test, we do the following replacements in equation 6.11: $\Delta_{i j \mu}^{v} \rightarrow \Delta_{0}^{v}$, and leave bond field and hole density inhomogeneous and unchanged from the nPDW solution. The chemical potential is adjusted in both tests to yield the correct average electron filling. Results for the lattice LDOS, form factors and spatial phase difference in first and second tests are shown in figures 3.9 (c), 3.10(a)(c), and 3.9 (d), $3.10(\mathrm{~b})(\mathrm{d})$ respectively. Comparing figure 3.2(c) with figures 3.9 (c) and (d), we find that the two sets of coherence peaks in the nPDW state lattice LDOS are indeed originating from the PDW, and that the CDW has an insignificant effect. Figure 3.10(a) shows that the $d$-form factor in the pure PDW state has the highest magnitude at the energy corresponding to one of the coherence peaks in the lattice $\operatorname{LDOS}\left(\Omega_{d}=0.16 t\right)$ and its bias dependence and 
overall scale is very similar to the nPDW state (figure 3.8(a)). However, when PDW order is artificially set to zero then $d$-form factor acquires a bias dependence and scale which is very different from the nPDW state as evident from figure 3.10(b). The importance of the PDW is again manifested in figure 3.10(c) which shows that setting the charge density modulations to zero artificially has little effect on the spatial phase difference observed in the nPDW state (figure 3.9(a)). However, when the PDW order is set to zero then we get a very different bias dependence of spatial phase difference as evident from figure 3.10(d). Thus we conclude that the most significant features in the bias dependence of lattice LDOS, $d$-form factor and spatial phase difference in the nPDW state are originating from the pair field modulations.

\subsubsection{Discussion}

Within the inhomogeneous Gutzwiller approximation, for the parameters employed here, the uniform $d$-wave superconducting state has a lower energy than the charge ordered states at all doping levels. Thus then PDW is not the ground state of the $t-t^{\prime}-J$ model. However, the energy difference between the uniform state and charge ordered states is really small [40]. Thus, it is entirely plausible that other effects not included in the model such as disorder and electron-phonon interactions may stabilize these fluctuating charge ordered states [43, 126]. In fact, the short-ranged nature of these states, observed in STM [69] and resonant elastic x-ray scattering experiments [68], suggests that disorder might be playing an important role. Different local disorder environments may then also pin slightly different states, resulting in slightly different local LDOS patterns that can be identified in STM images, not just two different ladder-type domains, as is normally assumed. As pointed out, the evolution of the Gutzwiller factors with doping is responsible for the remarkable degeneracy of the various charge states shown in figure 3.2 (a) across the doping range. The energy splitting of these states above the homogeneous superconducting state remains almost the same across this range as well. Thus the addition of a magnetic field on the order of $10 \mathrm{~T}$ or $1 \mathrm{meV}$ per site can potentially stabilize long range charge order. It is tempting to conclude that the recent observation of charge order in YBCO with a 
large correlation length, at a magnetic field of order $30 \mathrm{~T}$ may be reflecting this effect [127, 128].

We find that at a given doping, nPDW states with different ordering wave vectors $\mathrm{Q}$ around $(0.3,0)$ exist. Keeping the same initial guess and changing the system size $(N \times N)$ results in charge ordered states with slightly different $Q=(Q, 0)$, since $\mathrm{Q}$ is a multiple of $1 / N$. However, LDOS, form factor and spatial phase difference results are insensitive to such small changes. All such states at nearby Q are extremely close in energy, and hence, at the level of the Gutzwiller approximation, we can not quantitatively address the doping dependence of the charge order wave vector. However, the bias dependence of the form factors and spatial phase difference is robust with respect to the change of ordering wave vector, band structure $\left(t^{\prime}\right)$ and doping. We always find a dominant $d$-form factor at higher energies and a shift of $\pi$ in the average spatial phase difference beyond a particular energy scale.

The analysis presented in the previous section, whereby PDW and CDW order were artificially suppressed independently, strongly suggests that PDW character is necessary to explain the spectral characteristics, in particular the bias dependence of the intra unit cell form factors and spatial phase difference in experimental measurements on BSCCO.

It is important to note further that the bias dependence of the form factors in the current theory is the clear result of electronic correlations in the $\mathrm{CuO}_{2}$ plane. It has been observed in X-ray spectroscopy that the plane in $\mathrm{YBCO}$, for example, buckles in a pattern of $\mathrm{O}$ displacements that mimics a $d$-wave form factor [129], and suggested that this structural pattern imprints itself on the local tunneling conductance. However, it is difficult to see how such a structural effect should be sensitive to the applied bias, as seen in experiment and predicted here. Nor is it clear why, in such a scenario, the other form factors can be stabilized in other bias ranges.

\subsection{Momentum space properties}

After we have obtained a quite profound agreement with experimental observations for the real space properties, starting form this subsection we will head to discuss our discoveries 
(a)

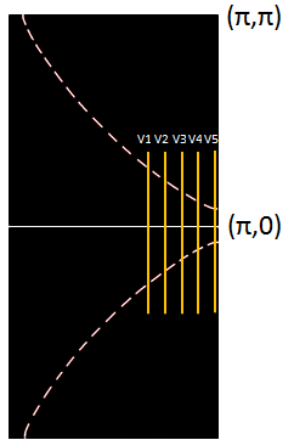

(d)

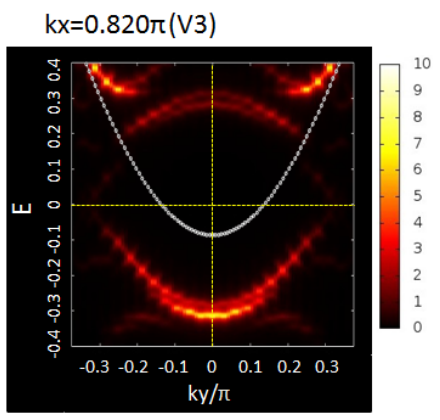

(b)

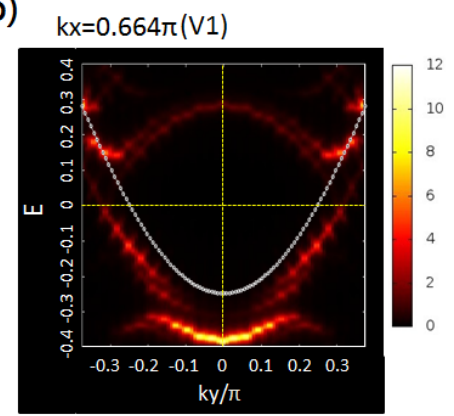

(e)

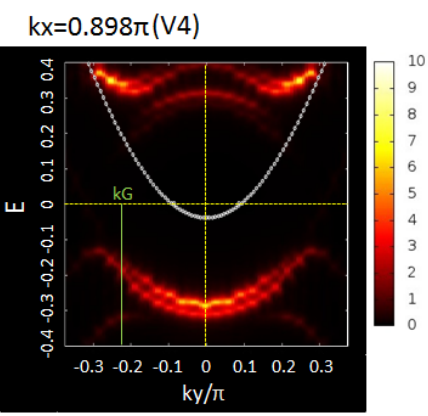

(c)

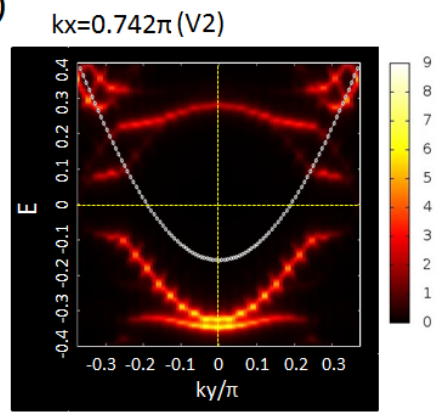

(f)

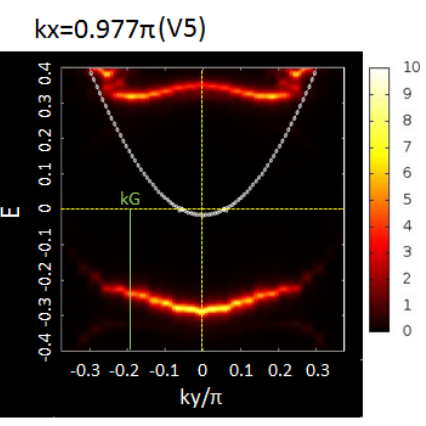

Figure 3.11: The quasiparticle spectra of a nPDW state calculated in a $32 \times 32$ lattice for hole concentration 0.125 : (a) the vertical cuts $(V 1-V 5)$ denote the y component of the momentums scanned from (b)(near nodal region) to (f)(anti-nodal region). (b)-(f): quasiparticle spectra weight for each cut as a function of $k_{y}$ with a fixed $k_{x}$ value shown above each figure.

for those features in the momentum space.

\subsubsection{Particle-hole asymmetry}

We will first discuss ARPES spectra for nPDW states. These states with incommensurate PDW, CDW, and bond order wave coexisting have a UPOP exhibiting a $d$-wave nodal like LDOS at low energy. Their energy dependence of the intra-unit-cell form factors with $s$, $s^{\prime}$ and $d$ symmetry and the spatial phase different agree well with the STS experiments as shown in the previous content.

The spectral density $A\left(k_{x}, k_{y}, \omega\right)$ of the above state is calculated by using Eq. 6.27 at $T=0$. We choose the width $\Gamma=0.01 t$ unless specially mentioned otherwise. In Fig. 3.11 (a) we scan the momentum space near the antinodal region $\left(k_{x}, k_{y}\right)=(\pi, 0)$ by having 5 vertical cuts $(V 1-V 5)$ perpendicular to the $k_{x}$ axis. The energy dependence of the spectral weight as a function of the y component of the wave vector $\left(k_{y}\right)$ for the 
(a)

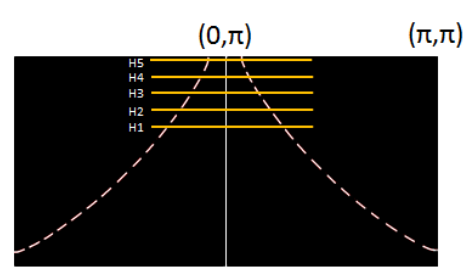

(d)

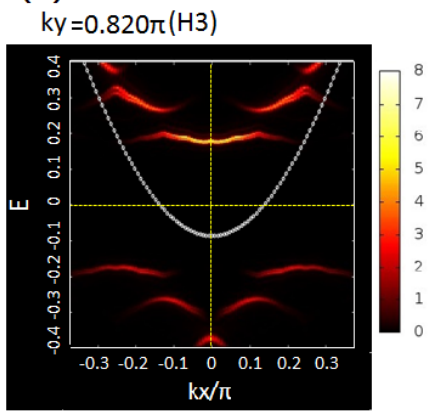

(b)

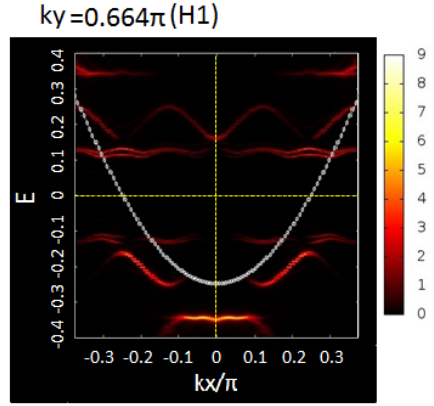

(e)

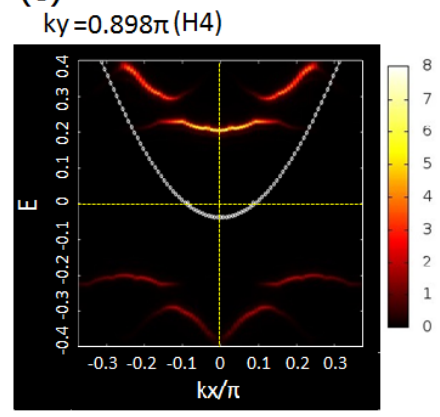

(c)

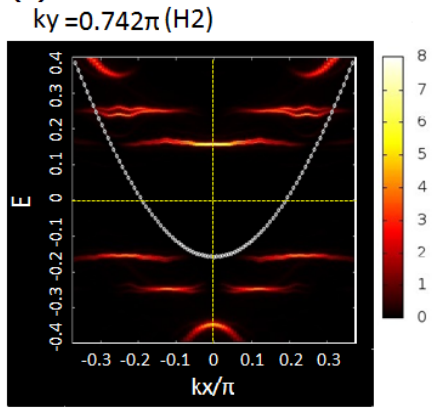

(f)

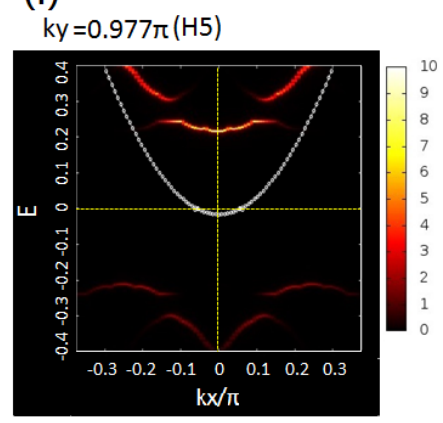

Figure 3.12: The quasiparticle spectra of a nPDW state calculated in a $32 \times 32$ lattice for hole concentration 0.125: (a) the vertical cuts $(H 1-H 5)$ denote the y component of the momentums scanned from (b)(near nodal region) to (f)(anti-nodal region). (b)-(f): quasiparticle spectra weight for each cut as a function of $k_{x}$ with a fixed $k_{y}$ value shown above each figure.

five cuts are shown in Figs. 3.11(b)-3.11(f). A very striking result is the complete lacking of particle-hole symmetry in the spectra as a BCS theory would have predicted. The white curves denote the dispersion of a uniform Fermi-liquid state(FLS) without pairing at dopant density 0.125 . The curve crossing zero energy are Fermi momenta $k_{F}$. The five cuts show that near the nodal region $V 1$ the gap at $k_{F}$ is small and increases substantially approaching toward the antinodes $V 5$. Most interesting part is the dispersion along each cut bends back after passing the minimum energy gap, which is determined by looking at bands above and below Fermi energy. These back-bending momentum $k_{G}$ moves away from $k_{F}$ as momenta approaching antinodes. The momentum $k_{G}$ is determined by using the energy distribution curves(EDCs). For the ARPES experiment, only the occupied states or the states with negative energies are measured, hence it cannot show the momenta with minimum gap but it can determine the back-bending momenta $k_{G}$. Indeed our result is very consistent with the experiments [82] showing this particle-hole asymmetry which 
is very different from usual BCS superconductors that $k_{G}=k_{F}$. It was first pointed out by Lee [39] that the difference between $k_{F}$ and $k_{G}$ and the way two approaches each other near nodal region is inconsistent with pure CDW either.

In the experiment, the spectra along $k_{x}$ direction is same as in $k_{y}$. This is likely due to the sample packed with $\mathrm{x}$ - and $\mathrm{y}$ - oriented short-range ordered unidirectional domains as seen in STS [59]. But here we only have one unidirectional nPDW, hence spectra along $k_{x}$ and $k_{y}$ are different. In Fig. 3.12(a), we scan five horizontal cuts(H1-H5) from near nodal region (b) to antinodal region (f). Comparing 3.11(b) with 3.12(b), we can see that there is no state at low energy for 3.12 (b). In general the minimum gaps are the same for $H 5$ cuts near $(0, \pi)$ and $V 5$ cuts near $(\pi, 0)$. It seems that the gap does not change much from 3.12 (d) to 3.12 (f) while it increases significantly from 3.11(d) to 3.11(f). The occupied bands are quite flat along the $k_{x}$ direction near $(0, \pi)$, and hence it is difficult to determine the bending vectors $k_{G}$. This kind of spectra near $(0, \pi)$ for a x-directional PDW is quite different from what one would expect for a pure CDW [39]. We also note that energy gap value of $0.21 t$ at the parallel direction (Fig. 3.12(f)) with respect to the modulation direction of density waves is about the same as in the perpendicular direction (Fig. 3.11(f)). This will be discussed further in the discussion section.

We have also considered anisotropy [40] in hopping $t_{x(y)}$ and $J_{x(y)}$ and if we decrease $t_{x}$ with respect to $t_{y}\left(J_{x} / J_{y}=t_{x}^{2} / t_{y}^{2}\right)$, then the nPDW state modulated in x-direction will no longer have a pure $d$-wave but a $s^{\prime}+d$ wave. The energy gap determined by the $H 5$ cut near $(0, \pi)$ increases but the value of $V 5$ cut near $(\pi, 0)$ is reduced. We will discuss this further in the Discussion section later.

\subsubsection{Two-gap in the SC phase}

The quasi-particle spectra in Fig. 3.11 and 3.12 also show an energy gap increasing as momentum approaches antinodal region. To compare with the result of ARPES experiment we shall use the EDCs to determine the gap. By taking a scan along a linear cut near the Fermi surface (the white curves in Fig. 3.11(a)), we can determine the $k$ value that has the smallest energy difference from chemical potential. This will determine the energy 

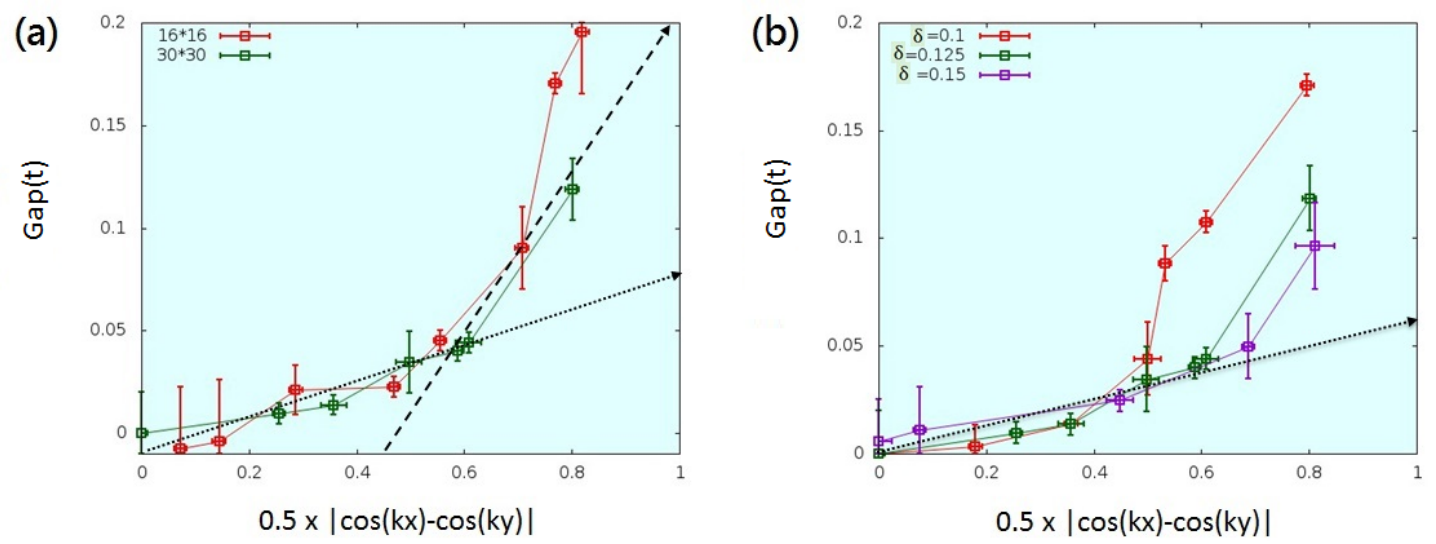

Figure 3.13: The gap value evolving from nodal to antinodal region for nPDW for (a) two different lattice sizes at doping 0.125; (b) different doping levels but same size $(30 \times 30)$. Red, green and purple lines are just guides for the eyes. The black dotted(dashed) line is a plotted pure $d$-wave(antinodal) gap with gap size about $0.075 \sim 0.08 t(0.2 \mathrm{t}$ ).

gap at this $k$ value. Going from the nodal direction at $k_{x}=k_{y}$ to the antinodal region $\left(k_{x}, k_{y}\right)=(\pi, 0)$, the energy gap is plotted as a function of $\left|\cos \left(k_{x}\right)-\cos \left(k_{y}\right)\right| / 2$ in Fig. 2 for nPDW states. In Fig. 2(a), at hole concentration 1/8, the red curve is obtained for a $16 \times 16$ lattice and green for $30 \times 30$. The vertical error bars are either determined by the width of the peak or by average of two nearby peaks with nearly the same magnitude. The horizontal error bars are due to the effect of discrete $k$ values. The results are essentially the same for these two different lattice sizes and in fact, there is also not much difference with the solution of $60 \times 60$ lattice. The vertical error bars are only slightly larger for the smaller $16 \times 16$ lattice. The slope of both curves increases as the momentum gets closer to the antinode. The dotted line in Fig. 2(a) indicates the linear setting of a pure $d$-wave pairing gap $\left(\Delta_{c}\left|\cos \left(k_{x}\right)-\cos \left(k_{y}\right)\right| / 2\right)$ near the nodal region. The plotted gap value $\Delta_{c}$ is much smaller than the gap at antinodal direction. The dashed line indicates a second gap near the antinodal region. Thus we have two different $d$-wave gaps near the node and antinode.

Furthermore we can examine the variations of these gaps with dopant concentration. In Fig. 3.13(b) the gaps are plotted for nPDW states calculated for a $30 \times 30$ lattice for three hole concentrations: red for $\delta=0.1$, green for 0.125 and purple for 0.15 . As hole concentration decreases, the gap at the antinodal region gets larger while deviation from the dotted line starts closer to the nodal $k$. The value of the gap at antinode could reach 
$0.2 t$ or about $80 \mathrm{meV}$ just as in the experiments [21]. At larger doping these two $d$-wave gaps seem to approach each other as a single gap which is expected in the usual BCS state. Due to the finite size effect, we cannot determine if the $d$-wave like gap for the three hole concentrations in Fig. 3.13(b) are exactly the same, but it looks close enough and with a value about $0.08 t \sim 32 \mathrm{meV}$ for $t=0.4 \mathrm{eV}$. In Fig. 3.13 we used a constant $\Gamma=0.01 t$, but the result is insensitive to the choice of the $\Gamma$ in calculating the spectra density. This is very consistent with ARPES results shown in Ref. [21, 130]. They found the gap value about $39 \mathrm{meV}$ near the nodal region for several different hole concentrations.

So far we have discussed the gaps and spectral density of the superconducting nPDW states. The state is quasi-periodic in the sense that it has several periods mixed but the dominant one is near $4 a$. The intra-unit-cell form factors are dominated by $d$-symmetry. The pair density modulation is mostly dominated by a vector $Q_{p}$, and the CDW has mainly a peak at $2 Q_{p}$ and also a peak at $Q_{p}$.

\subsubsection{Finite temperature IPDW states}

All the states discussed above are SC with a net $d$-wave UPOP. These were obtained by solving the mean-field $\mathrm{BdG}$ equations self-consistently at $T=0$. We recall that the strong correlation effect of Mott physics is originated from the very large on-site Coulomb repulsion or Hubbard $U$. This effect is translated into a Gutzwiller projection operator to prohibit double occupancy of electrons at each lattice site in the $t-t^{\prime}-J \operatorname{model}(\mathrm{Eq}$. 6.1). By following the GWA [56], we replace these projection operators by Gutzwiller factors, which are functions of hole density. Here we will make an intuitive assumption that these Gutzwiller factors remain unchanged at temperatures much smaller than the relevant energy scale $t$ and $J$ which are of order $0.4 \mathrm{eV}$ and $0.12 \mathrm{eV}$, respectively. Thus the BdG equations are easily generalized to finite temperatures and we could again find self-consistent solutions at finite $T$. Details are already discussed in the Methods section. Here we will present the results.

In Fig. 3.14, the average or net UPOP calculated for a lattice of $30 \times 30$ with doping $0.125,0.15$, and 0.16 are plotted as a function of $T / t$, shown in green, blue and red 


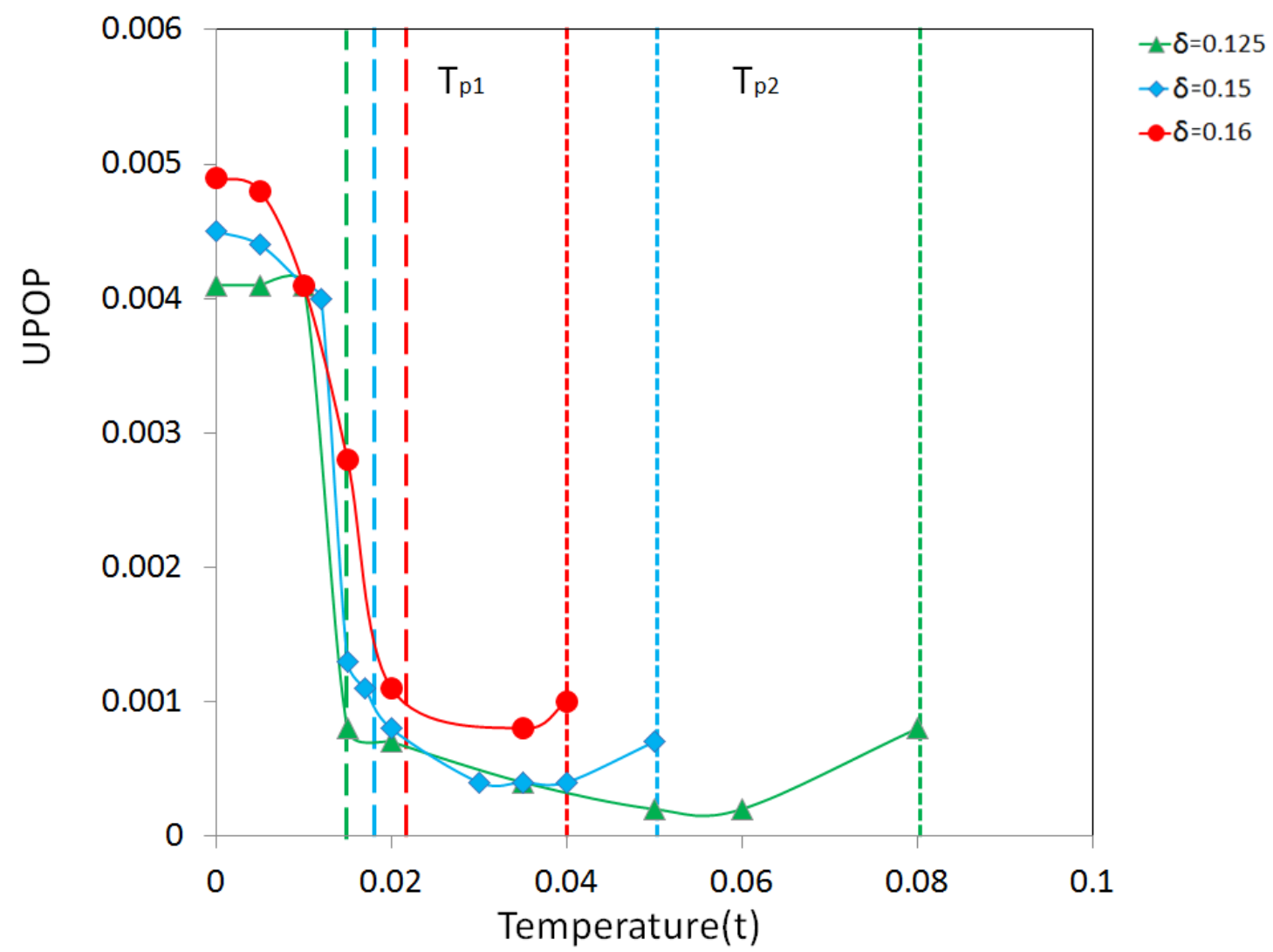

Figure 3.14: UPOP vs temperature for $\delta=0.125,0.15$, and $0.16 . T_{p 1}$ and $T_{p 2}$ for each case are marked with different dotted lines of the same colors. The lattice size is $30 \times 30$.

marks. $T_{p 1}$ and $T_{p 2}$ for the three hole concentrations are also denoted. For simplicity $T_{p 1}$ is determined when the magnitude of UPOP reaches about 0.001 . The three curves are quite similar except that near $T=0, x=0.16$ has the largest pairing order and also the largest value of $T_{p 1}$; however its $T_{p 2}$ is the smallest. The meaning of $T_{p 2}$ where no PDW exists becomes more clear when we examine its doping dependence. For $T>T_{p 2}$, the phase is actually a uniform $d$-wave state without modulations of charge and pairing. We already showed that the nPDW state and all other CDW or SDW states have a slightly higher energy than the uniform BCS state for the $t-J$ and $t-t^{\prime}-J$ model. If we include other interactions like long range Coulomb interaction or a weak electron-phonon interaction [42, 47], these density wave states could become lower in energy. Even if we only consider $t-t^{\prime}-J$ model, these nPDW states are stable solutions at local minimum, until they no longer exist at $T_{p 2}$ as shown in Fig. 3.14. This new IPDW state between $T_{p 1}$ and $T_{p 2}$ has almost zero UPOP but still large incommensurate modulations of charge density, 

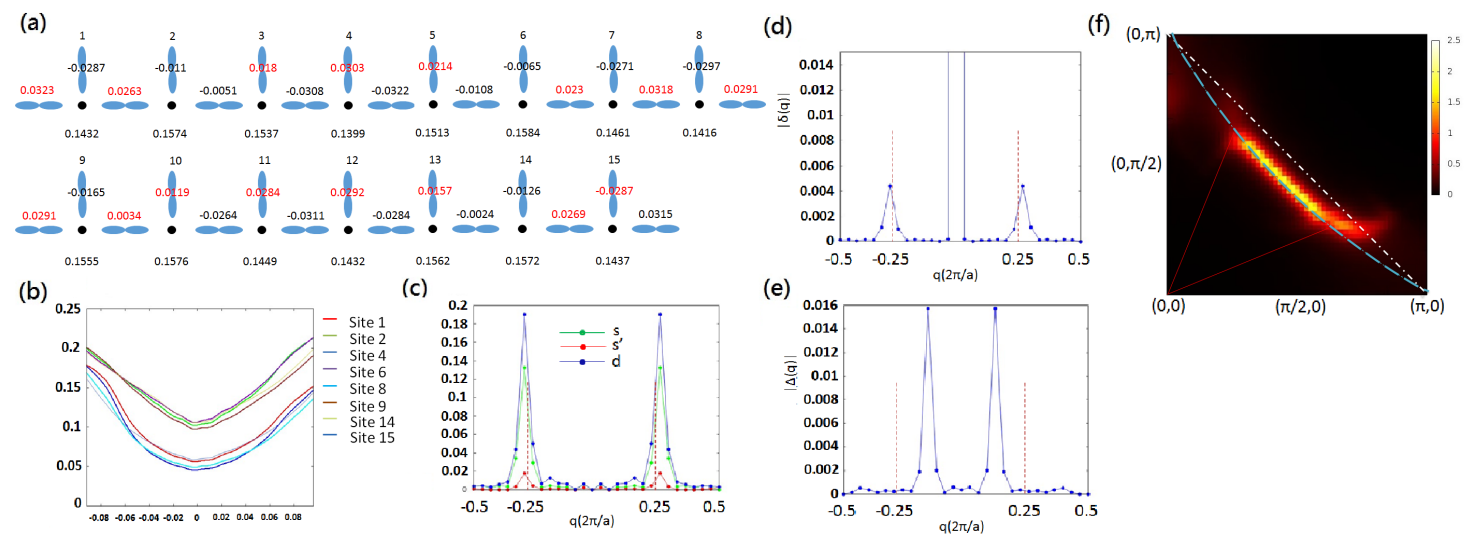

Figure 3.15: Properties of IPDW. (a) The real space modulation of IPDW. The red and black numbers on each bond denote the values of pairing order and the number at each site (black dots) is the hole density. (b) The LDOS for sites near the domain wall(2, 6, 9, 14 in (a)) and in the middle of nearby domain walls $(1,4,8,15$ in (a)). (c) Different form factors and (d)(e) Fourier transform of hole density(d) and pairing order(e). The red vertical dashed lines mark $|q|=0.5 \pi / a$ corresponding to period $4 a$. Quasiparticle spectra with zero energy in $k$ space for IPDW in $30 \times 30$ lattice sites at $T=0.035 t$ are shown in (f) for $\delta=0.15$. The cyan dotted curve is the Fermi surface of Fermi liquid state with the same doping level. $\Gamma$ used here is equal to $0.25 \sqrt{E^{2}+T^{2}}$ [2].

pairing order and bond order.

We will introduce the real space properties of IPDW now. The pattern of pairing order at each bond and hole density for an IPDW state with $\delta=0.15$ at $T=0.035 t$ is shown in Fig. 3.15(a). The hole density is maximum at sites, e.g. 2, 6 and 10 at the pairing boundary where the paring order changes sign. The LDOS at a few selected sites are shown in Fig. 3.15(b). There is a finite constant LDOS near zero energy and it is not nodal like as the usual $d$-wave SC and nPDW states. The Fourier transform of the intra-unit-cell form factor, hole density $\delta_{i}$ and pair field $\Delta_{i j}$ are shown in Figs. 3.15(c)3.15(e). Both the modulation wave vector of the bond order wave and CDW, shown in Figs. 3.15 (c) and 3.15 (d), respectively, are $2 Q_{p}=0.52 \pi / a$, while the pairing modulation is dominated by $Q_{p}=0.26 \pi / a$ as shown in Fig. 3.15(e). Most of the properties of this IPDW state are similar with nPDW state except three distinctions: a negligible net UPOP, a finite Fermi arc as shown in Fig. 3.15(f) and FT of charge density has no peaks at $Q_{p}$ [37, 88]. The color legend represents the spectral weight of these $k$-points on the arc in Fig. 3.15 (f). Notice the arc is asymmetric with respect to exchanging $k_{x}$ and $k_{y}$ as the modulation along $\mathrm{x}$-direction breaks the $\mathrm{x}$ and $\mathrm{y}$ symmetry. At the antinodal region the 

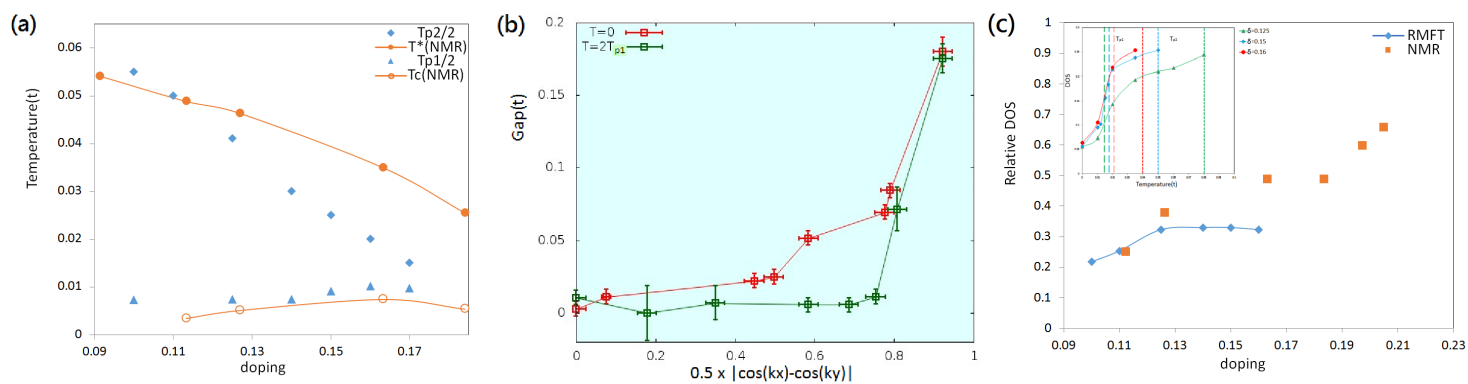

Figure 3.16: (a) Doping dependence of $T_{p 1}$ and $T_{p 2} . T_{p 1} / 2$ and $T_{p 2} / 2$ are shown with the blue triangles and diamonds respectively. The results from NMR [3] are also shown for comparison. We choose $0.1 t \sim 464 K$. (b) The gap values scanned along the Fermi surface at $T=0$ and $2 T_{p 1}$ for $\delta=0.15$. (c) Doping dependence of the relative DOS between IPDW and FLS $\left(D O S_{I P D W} / D O S_{F L S}\right)$ at $T=0.035 t$. The experimental data from [3] for $T=0$ is also plotted for comparison. The inset shows DOS of IPDW vs temperature for $\delta=0.125,0.15$, and 0.16 . $\Gamma$ we used here is $0.25 \sqrt{E^{2}+T^{2}}$ [2].

Fermi surface is gapped out similarly as the nPDW states.

In Fig. 3.16(a), $T_{p 1}$ and $T_{p 2}$ are plotted as a function of doped hole concentration with the blue triangles and diamonds respectively. We also plotted the PG phase temperature $T^{*}$ determined from the NMR measurement [3] for $\mathrm{Bi}_{2} \mathrm{Sr}_{2-x} L a_{x} \mathrm{CuO} \mathrm{O}_{6+\delta}$ in red color by taking $t$ to be $0.4 \mathrm{eV} . T_{p 1}$ follows a dome shape and has a maximum at hole concentration 0.16. The steep suppression of $T_{p 2}$ with doping is similar with the PG temperature $T^{*}$, and the values are also close if we reduce $T_{p 2}$ by about a factor of 2 . This is not surprising as we have neglected the quantum fluctuation effect in this mean field theory [131], and we also have assumed the Gutzwiller factors to have no $T$ dependence. Also note that so far we have only considered long-range-ordered solutions and have neglected solutions of random $x-$ and $y$ - oriented domains with short-range IPDW states. Since IPDW state has a Fermi arc as shown in Fig. 3.15(f), we expect the gap should vanish at the Fermi surface near the nodal region. In Fig. 3.16(b) the gap value along the Fermi surface is plotted for $T=0$ (red squares) and $T=2 T_{p 1}$ (green squares). This is very close to what is measured on BSCCO by ARPES [130]. The gap at antinode is essentially unchanged when the state changes from nPDW to IPDW. This is not surprising, since the antinode is still much larger than the temperature.

A very important property of the PG phase is the Knight shifts measured by NMR [3]; it shows that the DOS in the PG phase increases slowly with doping but is less than half 
of the DOS of the normal state for $T>T^{*}$ until it is near the critical doping about 0.2 where the PG phase disappears. It is also found that the Knight shift or DOS varies with temperature by less than $10 \%$ during the PG phase.

In the inset of Fig. 3.16(c), DOS is plotted as a function of temperature for three hole concentrations. Here we have assumed the width $\Gamma$, used in the spectra density calculation, is of the form $\Gamma=0.25 \sqrt{E^{2}+T^{2}}$ [2]. The DOS is calculated at zero energy(within an energy range of $\pm 0.004 t$ ) by averaging the LDOS at all sites. The DOS are all quite small and almost the same at $T=0$ but it increases significantly at $T_{p 1}$. The DOS values between $T_{p 1}$ and $T_{p 2}$ increase with doping. This is likely due to the fact that the length of Fermi arc increases with doping. The variation of DOS with $T$ between $T_{p 1}$ and $T_{p 2}$ for these three hole concentrations are also near $10 \%$ as in experiments. In Fig. 3.16(c), the ratio of DOS between the IPDW states and the FLS is plotted as a function of dopant concentration at $T=0.035 t$. Not only the doping dependence is very close to the experimental data [3] shown as red symbols, the values are also close to the measured results. It is difficult for us to obtain solutions above dopant concentration 0.17 as $T_{p 2}$ and $T_{p 1}$ are very close(Fig. 3.16(a)). When the dopant concentration is above 0.18 , we have no nPDW solution at $T=0$ and no IPDW state at finite $T$. Thus we would recover the full Fermi surface and the relative DOS should be 1 . For real materials this happens at concentration 0.2 instead of 0.18 .

The quasi-particle spectra of the IPDW state is very similar with those for nPDW state. In fact even for DCnPDW they all share similar profile. In Fig. 3.17, we demonstrate the spectra at $(\pi, 0)$ and $(0, \pi)$ for three different patterns chosen: nPDW at 30 and 32 lattice size, and DCnPDW at 36 lattice size. It is clear that, as mentioned earlier, the spectra at antinodes $(\pi, 0)$ and $(0, \pi)$ are quite different but the gap values are very similar [4]. More interestingly, although there are three different states, their quasi-particle spectra are also very close to each other. Even we increase the temperature, there is still not qualitative difference for the spectra, but only that the gap values decrease a bit as temperature rises. One need to note that at $T=0.035 t$ and $T=0.05 t$ the states have already evolved to IPDW states. Once again, this result suggests that although there are numerous pos- 


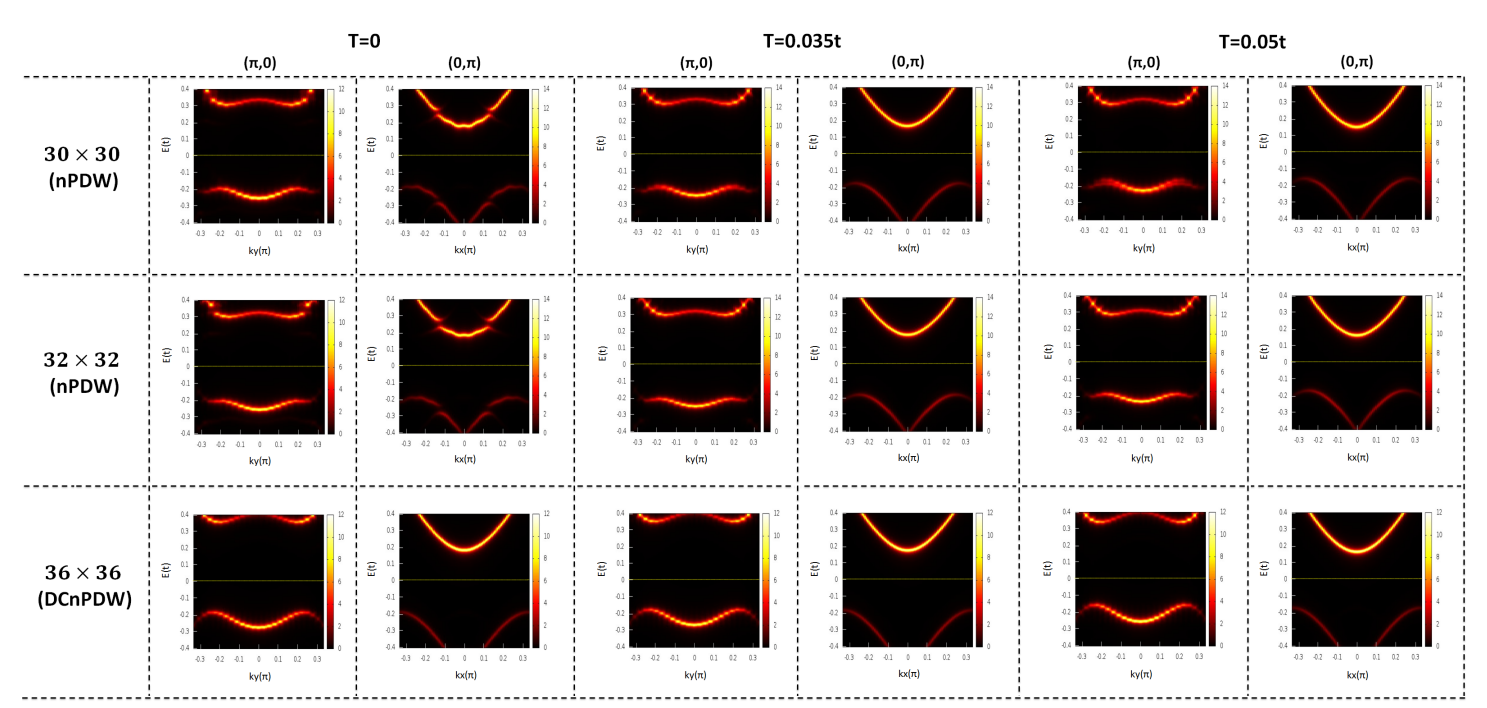

Figure 3.17: We list several quasi-particle spectra at antinodes $((\pi, 0) /(0, \pi))$ for three different patterns at different temperatures. Although marked as nPDW in the first column, the patterns become IPDW at $T=0.035 t$ and $T=0.05 t$. However their spectra do not change much and the differences of gap values at $(\pi, 0)$ and $(0, \pi)$ are within $10 \%$ [4].

sibilities of having a commensurate, (quasi-)incommensurate, or discommensurate state w/wo UPOP, the deeper cause is alway the same: strong correlated Mott physics with the Gutzwiller factors.

\subsubsection{Discussion}

Assuming that the Gutzwiller factors which take into account the renormalization effect of the strong correlation physics could have very small temperature dependence below room temperatures, we then generalize the renormalized mean-field theory to finite temperatures to study the prediction of the $t-t^{\prime}-J$ model.

At low-temperature SC phase with a finite UPOP, a special self-consistent solution, the nPDW state first found by us, is shown to have two $d$-wave pairing gaps as found by the ARPES. The smaller the doping, the larger is the gap magnitude at antinodes, but the nodal gaps are almost same for different dopings. The larger particle-hole asymmetry reported near the antinodal region is also well produced by the calculated spectra function. This nPDW state has a very special property that although it has a one-dimensional structure, the net pairing order or UPOP still has the four-fold $d$-wave symmetry. It is quite amazing that although the pairing value at each bond looks quite random (Fig. 3.3(a)), 
its average has an exact $d$-wave symmetry. The spectra at antinodes $(\pi, 0)$ and $(0, \pi)$ are quite different but the values are close to each other [4]. Combining together with previous works comparing our calculated LDOS and local spectra with the STS measurement, we have obtained a very consistent picture about experimental data for both spectra in real space and in momentum space for the superconducting phase.

Here it is worthwhile to make a special discussion that the nPDW state we chose is among many possible solutions with different periods. Fortunately most of them examined by us have very similar properties except that the periods of modulations could be different. In terms of energy the uniform $d$-wave SC state is the "true" ground state of the $t-t^{\prime}-J$ model within our RMFT. However, as we mentioned earlier, whenever other weak interactions, such as electron-phonon interaction, nearest neighbor or long range Coulomb force and impurities or defects, are added to the model [42, 47], the nPDW state could be stabilized. Even if we only consider pure $t-t^{\prime}-J$ model, these states are in their local minimum. Thus we could study its low energy excitations.

Another thing we like to point out is that experiments just like our theory also have found different kinds of CDW states. For the $\mathrm{La}_{2-x} \mathrm{Ba}_{x} \mathrm{CuO}$ family, the period of CDW decreases with doping while it increases for YBCO and BSCCO [19]. These two are called CDW1 and CDW2, respectively, in the review article [36]. There is also CDW3 or the magnetic field induced CDW. In this work we only concentrated on CDW2, which has no magnetic component. We believe CDW1 is probably the stripe state [47].

When the temperature is raised, the net UPOP in the nPDW state begins to decrease and it becomes negligible at certain temperature $T_{p 1}$. This behavior also supports our assumption that these states are at a local minimum. Then it changes into an IPDW state that still has incommensurate modulations of charge density, bond order and pairing order but without a net pairing order. Magnitude and modulation periods of all these three orders are quite similar to the nPDW state except that the FFT of the charge density does not have a peak at the wave vector of the pairing modulation as seen in nPDW [88]. In IPDW state, the modulation momentum of charge is twice of pairing. These states vary gradually with temperature until it reaches a higher temperature $T_{p 2}$ and there is no longer a solution 
with modulations of pairing. Quite unexpectedly $T_{p 2}$ actually decreases sharply as doping increases. Fig. 3.16(a) shows that $T_{p 2}$ is proportional to the PG temperature $T^{*}$ with an overestimation of at most a factor two for its values. This is quite satisfactory for a simple mean-field approach like ours. More discussion about this is given below.

Furthermore our analysis shows that the IPDW state near nodal region has a Fermi arc with a fraction of DOS of the full Fermi surface when there is no pairing. There is still a large gap at the antinodal region as shown in Fig. 3.16(b). The DOS or the length of Fermi arc increases with dopant concentration just as what were seen by ARPES [21] and NMR [3].

In our calculation we obtain the uniform $d$-wave $\mathrm{SC}$ state at $T$ greater than $T_{p 2}$. However as we mentioned earlier, this could be a consequence that we actually are at a local minimum and uniform SC state is the global minimum solution. We believe that if we consider solutions composed of randomly packed $\mathrm{x}$ - and $\mathrm{y}$ - oriented domains of these IPDW states, its large entropy would have a lower free energy than that of the uniform SC state. Thus the reappearance of the uniform $d$-wave $\mathrm{SC}$ state at high $T$ is indicating the limit of accuracy of our mean-field theory and it probably has no physical significance. As mentioned earlier, a much more accurate numerical work [55] than our mean-field result for Hubbard model at dopant 0.125 shows that uniform state is not the ground state. The stripes including SDW in addition to PDW and CDW are possible ground states for $U / t=12$ or less. For larger $U$ as is for $t-J$ model, antiferromagnetism is weaker and the nature of ground state is yet to be settled.

It should be emphasized that the IPDW state is also a SC FFLO [85, 86] state with finite momentum pairing if there is a phase coherence. But actually there maybe solutions with disordered fluctuating domains [132] with different charge density, phases and periods, etc. Variational Monte Carlo calculations have shown [43] that random stripe domains could be very competitive in energy in comparison with the long-range-ordered state. Furthermore the short-range-ordered domains of these IPDW states will have larger entropy and lower free energy. The PG phase is known to have strong vortex fluctuations [133, 134]. The inclusion of phases for these PDW states and their coupling with 
vortices [88, 93, 94] will provide a better and wholistic description of the PG phase. However, the PDW described here, we believe, should still be a basic entity included in these considerations to account for the spectra measured by experiments.

Another important issue we have not addressed is the effect of magnetic field on the PDW. Magnetic field-induced unidirectional CDW states have been reported below and above $T_{c}$ for $\mathrm{YBCO}[135,136,137]$. Some are even long-range ordered in 3D [127]. Somewhat different results are found in BSCCO family. Recent experiment on BSCCO has found bidirectional PDW or checkerboard of 8 unit cell period existing inside the vortex halo [138]. For $\mathrm{Bi}_{2} S r_{2-x} L a_{x} C u O_{6}$, NMR measurement [139] shows that an inplane magnetic field of $10 T$ is enough to induce long-range ordered CDW without spin components in the PG phase. Since such a small in-plane field does not have much effect on our nPDW or IPDW states, we believe these states are the ones observed in $B i_{2} \mathrm{Sr}_{2-x} L a_{x} C u O_{6}$. This is supported by the good agreement achieved in Fig. 3.16)(c) between the calculated DOS of our IPDW states and the Knight shift measurement in the PG phase by suppressing SC phase with high field [3].

\subsection{Some details}

In this section, we shall discuss several details, such as the method we used to determined $k_{G}$, the two gaps and Fermi arcs. We will also discuss the effect of using different $\Gamma$ in calculating the spectra density.

\subsubsection{Method to determine $k_{G}$}

We have mentioned that as the cut of spectra goes away from the node toward near the antinode, the momentum of $\operatorname{gap}\left(k_{G}\right)$ will also deviate from $k_{F}$. The way of determining $k_{G}$ will require the usage of energy distribution curves(EDCs). In Fig. 3.18(b), we show the spectra at $(\pi, 0)$ for $n$ PDW in the doping level of $0.15(30 \times 30$ lattice size $)$. Next to the spectra, we also put in a series of EDC cuts starting from the point $(\pi, 0)$ toward $(\pi, \pi)$. Just like the ARPES experiment, we can easily determine $k_{G}$ when the minimum gap is 

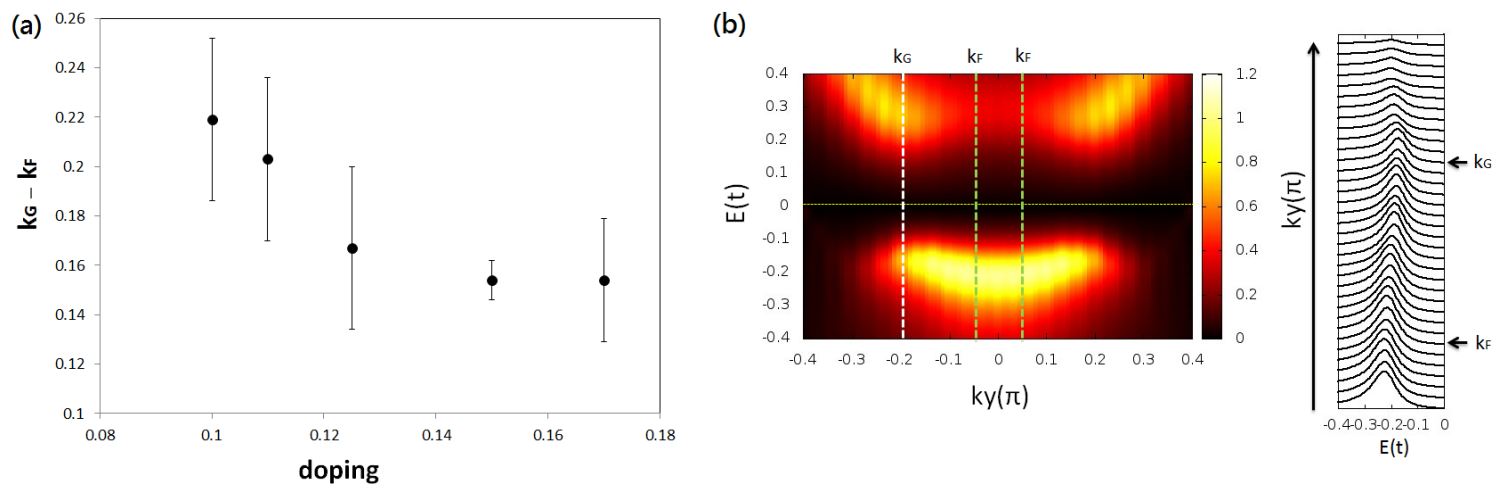

Figure 3.18: (a) A collection of several data points of $k_{G}-k_{F}$ vs doping at $k_{x}=\pi$. The way of determining the difference of $k_{G}$ and $k_{F}$ is shown in (b): $k_{G}$ determined by examining EDCs plotted from $k_{y}=0$ toward $k_{y}=p i$, for dopant concentration $0.15 . k_{F}$ is determined by Fermi liquid surface and marked along with $k_{G}$ on the EDC plot. The quasiparticle spectra is also shown with Gaussian width $\Gamma=\alpha|E|(\alpha=0.25)$ and marked with positions of $k_{G}$ and $k_{F}$.

reached by looking at the EDC cuts. Note that here we use $\Gamma=0.25|E|$. The difference between $k_{G}$ and $k_{F}$ as a function of doping is shown in Fig. 3.18(a). The difference becomes smaller as doping increases. This is expected since the gap approaches a pure $d$ wave gap as doping increases and particle-hole symmetry is recovered for the usual BCS superconductors.

\subsubsection{Two-gap plots}

Here we discuss the method used to determine gap values in Fig. 3.13 and 3.16(b), as well as their error bars. First we will explain that in fact there is only small difference if we utilize different ways of determining gap. In the above, all the values are determined by using EDCs and the horizontal error bars come from the finite size effect, which could be reduced if we further apply supercells with larger size, while the vertical error bars come from either the width of peaks(due to the choice of $\Gamma$ ), or the fact that there are actually several peaks coexisting. But in fact there are different ways of determining gap values and they will provide the same outcomes. For example, the quasiparticle spectra can be also used to determine the gap as explained earlier. The result are all the same no matter which way we decided to exploit. Fig. 3.19(a) put together two curves of gap values determined by EDC and quasiparticle spectra. One can see that these two lines are very 


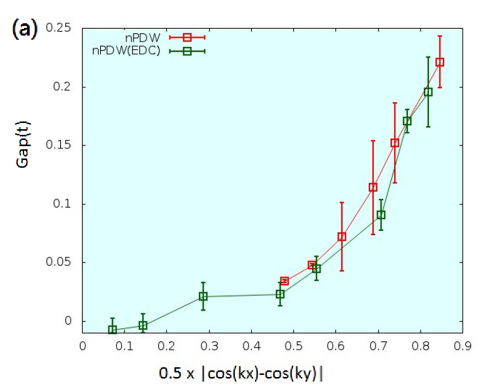

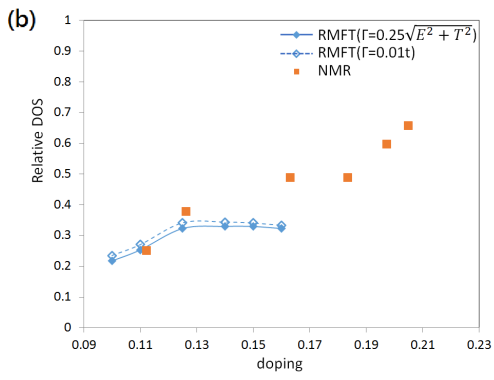

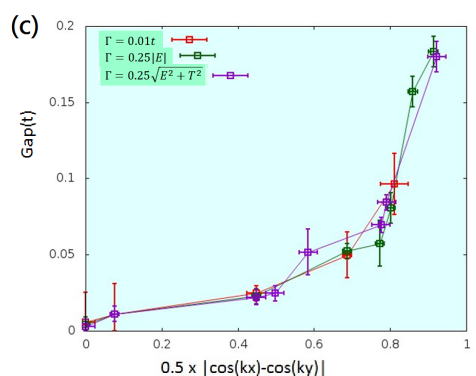

(d)

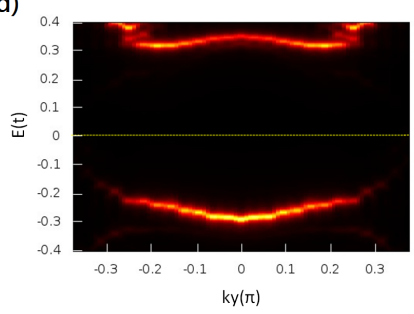

(e)

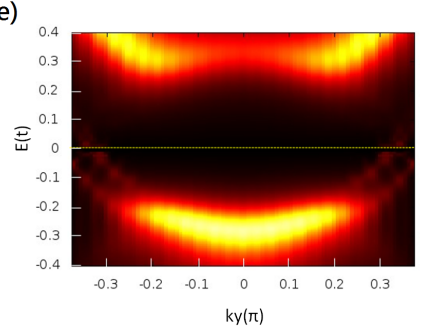

Figure 3.19: (a) Two-gap plot for nPDW at $\delta=0.125$ as shown in Fig. 3 in the main text but obtained from different approaches: red line is determined by the gap values shown by quasiparticle spectra but green line comes from EDCs. (b) Relative DOS as a function of hole concentration as in Fig. 3.16(c) in the main text but put together with two different $\Gamma$. The two blue lines are very close to each other. (c) Two gap plots determined by different $\Gamma$ for nPDW at $\delta=0.15$. One can see that these lines nearly overlap with each other. Figure (d) and (e) again show the quasi-particle spectra for nPDW at $\delta=0.125$ (for the $32 \times 32$ lattice) at $k_{x}=0.977 \pi$ but with different $\Gamma$ : (d) $\Gamma=0.01 t$ and (e) $\Gamma=0.25|E|$. Note that in fact (d) is identical as Fig. 3.11(f) in the main text. We can find that although these two figures look quite different due to the choices of $\Gamma$, important features such as location of $k_{G}$ are still the same, only that in (e) the spectra bands are broadened due to larger $\Gamma$.

close and even if there are small differences, they are within the error bars.

\subsubsection{Choices of $\Gamma$}

We mentioned that the width $\Gamma$ is chosen as different values for better demonstration in different plots. But in fact we have done a series of analysis showing that there is no qualitative difference in choosing $\Gamma$ to be a constant as $0.01 t$ or as $0.25 \sqrt{E^{2}+T^{2}}$. In Fig. 3.19(b) we plotted the same figure as Fig. 3.16(c). But here we include also the curve using $\Gamma=0.01 t$. One can see clearly that there is only small quantitative differences between two blue curves. Our second proof is to investigate the two-gap plots as Fig. 3.13 and 3.16(b), with different choice of $\Gamma$. In Fig. 3.19(c) we show the curves of gap values for $\mathrm{nPDW}$ at $\delta=0.15$, but under different choices of $\Gamma$. We can see that those three 
curves are nearly the same within error bars.

Last but not least, we also need to check the consistency of quasi-particle spectra. In Fig. 3.19(d) and (e), we plotted the same spectra but with different $\Gamma$, one with $\Gamma=$ $0.01 t(\mathrm{~d})$ and another with $\Gamma=0.25|E|(\mathrm{e})$. If we discount the broadening of Fig. 3.19(e), 3.19 (d) and 3.19 (e) have the same $k_{G}$.

\subsubsection{Fermi arcs and LDOS}

(a)

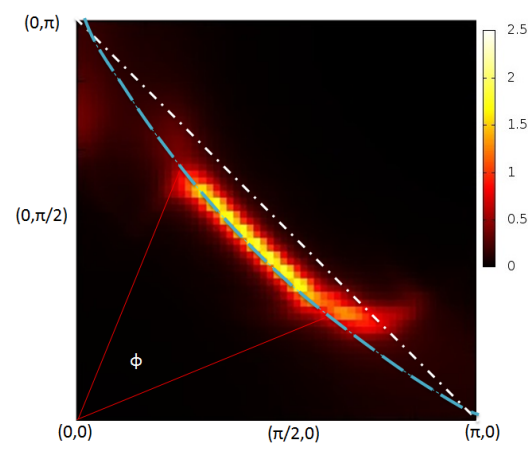

(c)

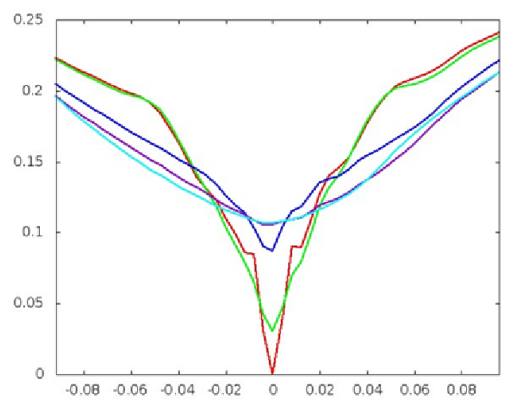

(b)

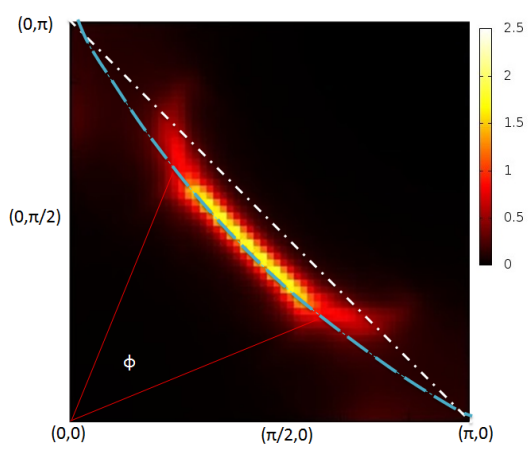

(d)

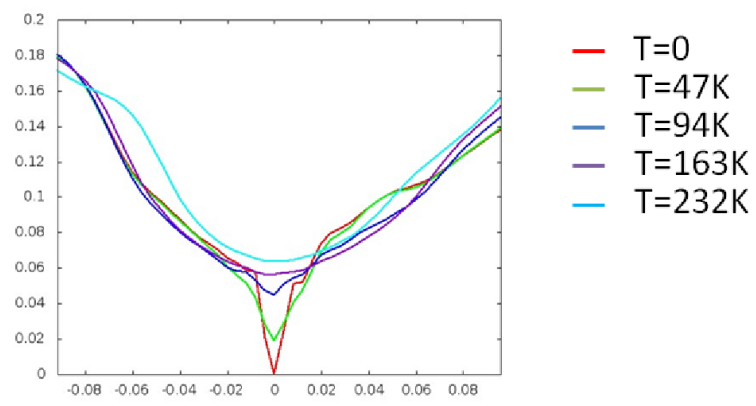

Figure 3.20: (a) and (b) Zero energy quasiparticle spectra in k space before(a) and after(b) taking average of $\mathrm{x}$ - and $\mathrm{y}$-directions PDW. (a) is the same as Fig. $3.15 \mathrm{f}$ and we put it here again for the reason of comparison. Clearly, (b) looks more like the observation by experimental groups. (c) and (d) LDOS at sites near(c) and away from(d) domain walls at different temperatures for nPDW(IPDW) at $\delta=0.15$. $\Gamma$ used here is equal to $\alpha \sqrt{E^{2}+T^{2}}(\alpha=0.25)$. All figures shown here are of $30 \times 30$ lattice size. Its $T_{p 1}$ is around $90 \mathrm{~K}$.

We have shown that the UPOP of nPDW is decreasing when temperature rises. The resulting pattern is called IPDW by us, which is also a PDW phase but UPOP is close to zero. Fig. 3.15(f) plots the zero energy quasiparticle spectra weight in momentum space and it reveals the feature of the so-called Fermi arc. However, in experiments arcs usually have $\mathrm{x}$ and $\mathrm{y}$ rotational symmetry. That is because the experimental detection scans over 
a region of materials that contains domains with modulations in both $\mathrm{x}$ and $\mathrm{y}$ direction. Therefore the resulting arcs would have the rotational symmetry. In order to compare with their results, we took average of $\mathrm{x}$ and $\mathrm{y}$ axis of our arcs and replotted it. The resulting figure is as Fig. 3.20(b), which looks more like the experimental data.

One of the main differences upon having UPOP or not is to look at the LDOS. Since our nPDW possesses $d$-wave UPOP, its LDOS will have a v-shape feature near the Fermi energy. However, for IPDW there is no UPOP and therefore the DOS at Fermi energy should be non-zero. Consequently, to further confirm the vanishing UPOP, we compare the LDOS of sites near and away from domain walls in Fig. 3.20(c) and 3.20(d), respectively. LDOS for five different temperatures are shown and the state remains nPDW for $T=0$ and $47 K$ but becomes IPDW at $T=94 K, 163 K$, and $232 K(0.1 t \sim 464 K)$ because of the disappearance of UPOP. According to the LDOS plots, it is also clear that the v-shape feature disappears gradually as temperature rises, confirming that the node has changed into an arc in IPDW state. 


\section{Chapter 4}

\section{Results II - Correlated Electrons Under}

\section{Magnetic Field}

By using the RMFT with an additional phase, we have repeated the same process mentioned earlier and solved for several self-consistent solutions. Table 4.1 shows the parameter sets we have used in the RMFT self-consistent calculations and we have plotted a simple phase diagram in Fig. 4.1 for better demonstration. For simplicity, we choose to work on a square lattice geometry with periodic boundary conditions and a $4 \times 4$ magnetic sublattice is used to encode an integer number of flux quanta. Hence, the flux per plaquette can be chosen as $\Phi=p / q$ with $q=16$ and $p$ any integer, giving a total number $N_{\Phi}=\Phi N_{\mathrm{s}}$ of magnetic flux quanta piercing the whole torus surface, where $N_{\mathrm{s}}$ is the number of lattice sites. The particle filling $\rho$ is equal to $\frac{N_{\mathrm{e}}}{2 N_{\mathrm{s}}}$, with $N_{\mathrm{e}}$ being the number of electrons. The doping with respect to the half-filled Mott insulator is $\delta=2\left(\frac{1}{2}-\rho\right)$. Because of particle-hole symmetry we can restrict to $\delta>0$. The filling fraction $\nu=\rho / \Phi$ indicates the ratio of Landau levels filled in the corresponding non-interacting picture. Clearly it is relevant for zero-magnetization systems, denoted by $S=0$ in Table 4.1. In contrast, a fully polarized (FP) GS would instead be "adiabatically" connected to a noninteracting (spinless) fermion system at filling fraction $\nu^{*}=2 \nu=2 \rho / \Phi$. The last column of Table 4.1 contains the information about the unit cell characterizing a possible (spontaneous) ordering for each state. Notice that the largest cluster size that can be reached with 


\begin{tabular}{|c|c|c|c|c|c|c|c|c|}
\hline$\rho$ & $\Phi$ & $\nu / \nu^{*}$ & $N_{\mathrm{s}}$ & $N_{\mathrm{e}}$ & $N_{\Phi}$ & $S$ & Unit Cell & Instabilities \\
\hline \hline $7 / 16$ & $7 / 16$ & 1 & $16 \times 16$ & 224 & 112 & 0 & $1 \times 1$ & None \\
\hline $7 / 16$ & $5 / 16$ & $7 / 5$ & $16 \times 16$ & 224 & 80 & 0 & $2 \times 2$ & BDW/PDW \\
\hline $7 / 16$ & $3 / 16$ & $7 / 3$ & $16 \times 16$ & 224 & 48 & 0 & $4 \times 4$ & CDW, BDW/PDW \\
\hline $7 / 16$ & $1 / 16$ & 7 & $16 \times 16$ & 224 & 16 & 0 & $\sqrt{2} \times \sqrt{2}$ & SC \\
\hline $7 / 32$ & $7 / 16$ & $1^{*}$ & $12 \times 12$ & 63 & 63 & $F P$ & $1 \times 1$ & None \\
\hline $1 / 8$ & $1 / 4$ & $1^{*}$ & $12 \times 12$ & 36 & 36 & $F P$ & $2 \times 2$ & CDW, BDW \\
\hline $1 / 8$ & $7 / 16$ & $4 / 7^{*}$ & $12 \times 12$ & 36 & 63 & $F P$ & $1 \times 1$ & None \\
\hline $1 / 16$ & $5 / 16$ & $2 / 5^{*}$ & $12 \times 12$ & 18 & 63 & $F P$ & $4 \times 4$ & CDW, BDW \\
\hline $1 / 16$ & $7 / 16$ & $2 / 7^{*}$ & $12 \times 12$ & 18 & 45 & $F P$ & $1 \times 1$ & None \\
\hline
\end{tabular}

Table 4.1: Parameter sets used in the following subsections. $N_{\mathrm{s}}, N_{\mathrm{e}}$, and $N_{\Phi}$ are the site, electron and flux numbers used for performing RMFT (those for the ED on a $4 \times 4$ cluster are obtained from a simple rescaling). Sets are listed with decreasing electron filling from top to bottom. The GS is either a singlet $(S=0)$ or fully polarized (FP), i.e., the total spin is $S=\frac{N_{\mathrm{e}}}{2}$ (in that case $\nu^{*}=2 \nu$ is listed and marked with an asterisk). The supercell associated to a possible spontaneous (charge or bond) ordering is also shown. $1 \times 1$ means the GS is uniform. CDW, BDW, and PDW stand for charge, bond, and pairing density wave. SC means staggered current modulation. For $\rho=7 / 16$ and $\Phi=5 / 16$ or $3 / 16$, including (d-wave) superconducting order in addition to CDW/BDW order gives a PDW self-consistent solution with lower energy. For $\rho=1 / 8$ and $\Phi=1 / 4\left(\nu^{*}=1\right)$, the $2 \times 2$ modulation is induced by a staggered potential. Otherwise, translation symmetry breaking (if any) occurs spontaneously.

ED is $4 \times 4$ corresponding to a unique magnetic unit cell. In that case, the corresponding flux and electron numbers $N_{\Phi}=16 \times \Phi$ and $N_{\mathrm{e}}=32 \times \rho$ need to be integers. In the two following subsections, we shall review the properties of the various phases found, the uniform and modulated flux states and the ferromagnetic FP phases, as can be inferred from the properties listed in the last two columns of Table 4.1 .

\subsection{Uniform and modulated singlet flux phase}

The first phase of interest which could be realized in this Hamiltonian is the Anderson, Shastry, and Hristopoulos (ASH) state [140]. It is also called CFP because of its commensurability condition between the flux and electron filling [48]. It has been shown that these states can be formally written in the quantum spin liquid form, the singlet bond amplitudes of which break the lattice translational symmetry [117], and their order of commensurability with the lattice unit length is closely related to the hole density [114, 115, 117]. The 


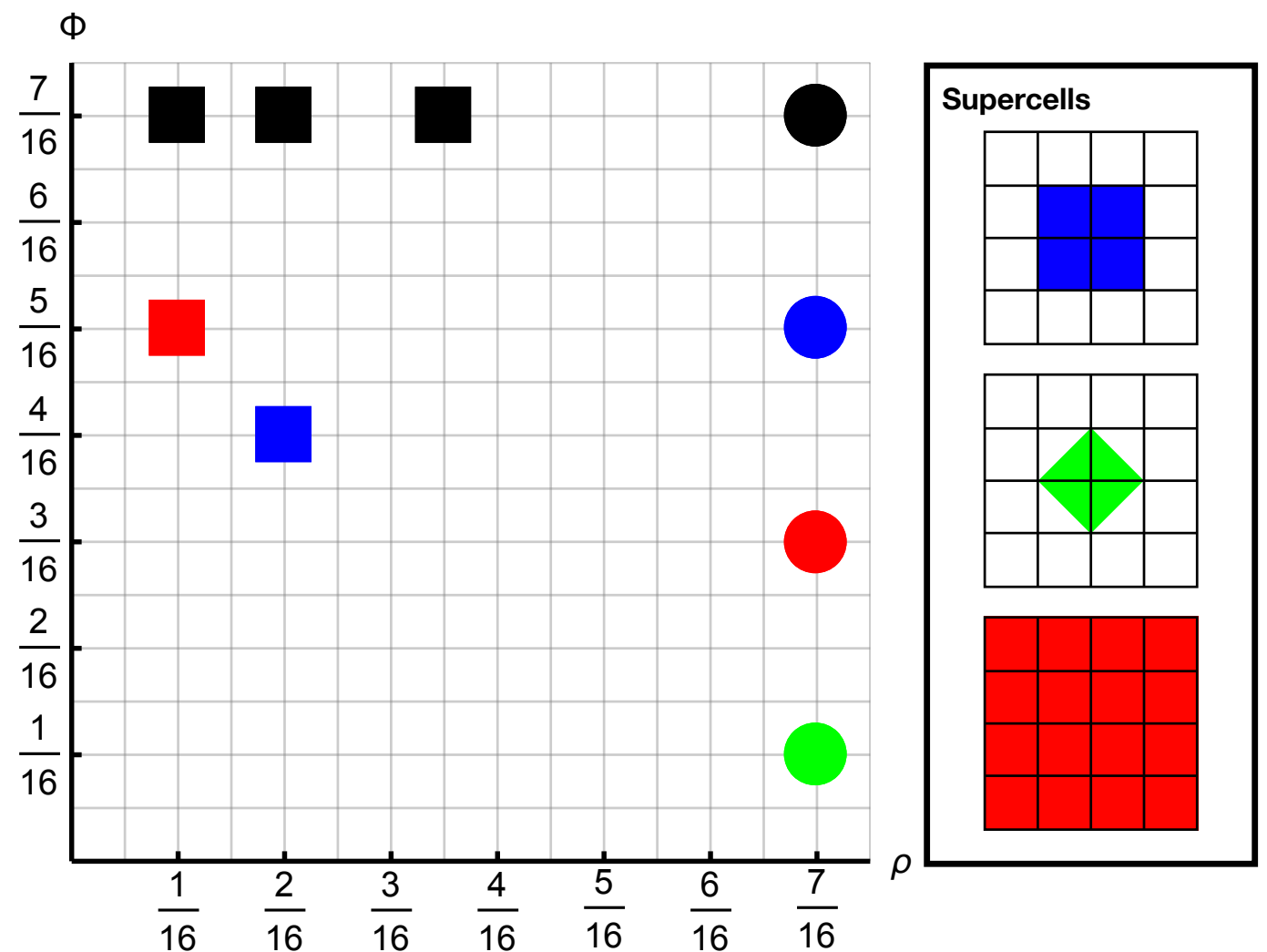

Figure 4.1: "Phase diagram" vs electron filling $\rho$ and magnetic flux $\Phi$ showing the various phases presented in Table 4.1. Circles are non-polarized (singlet) states while squares represent ferromagnets. Black symbols correspond to uniform solutions. Red, green, and blue symbols encode symmetry-breaking supercells of size $4 \times 4, \sqrt{2} \times \sqrt{2}$, and $2 \times 2$ (with staggered potential for $\Phi=1 / 4$ ) respectively.

stability of the CFP with varying flux, first discussed in Refs. [48, 114], will be revisited here.

In this section we fix the electronic fraction to be $\rho=7 / 16=0.4375$ and study how the states evolve with changing flux. This corresponds to a weakly-doped Mott insulator with a doping $\delta=2\left(\frac{1}{2}-\rho\right)=1 / 8$, i.e., two holes per magnetic $4 \times 4$ supercell. Within this choice of parameters, a uniform CFP has only been found for $\Phi=\rho=7 / 16$ (first line of Table 4.1). For the same doping and other commensurate values of the flux, $\Phi=p / 16 \neq \rho$ with $p$ an odd integer, singlet phases exhibiting lattice symmetry breaking patterns have been found, as is the case for the parameters corresponding to the second, third and fourth lines of Table 4.1. These patterns could correspond to a modulation of the (site) charge density and/or a modulation of the (real) bond hopping amplitude, which are the CDW or BDW, respectively. CDW and BDW orders may or may not coexist (compare second 


\begin{tabular}{|c|c|c|c|c|c|c|}
\hline$\rho$ & $\Phi$ & $\nu / \nu^{*}$ & $E_{0}$ & $E_{\text {kin }}$ & $E_{\text {pot }}$ & $C_{\text {RMFT }}$ \\
\hline $7 / 16$ & $7 / 16$ & 1 & $-8.945 \mathrm{t}$ & $-6.539 \mathrm{t}$ & $-2.405 \mathrm{t}$ & 2 \\
\hline $7 / 16$ & $5 / 16$ & $7 / 5$ & $-8.119 \mathrm{t}$ & $-5.882 \mathrm{t}$ & $-2.238 \mathrm{t}$ & 2 \\
\hline $7 / 16$ & $3 / 16$ & $7 / 3$ & $-7.632 \mathrm{t}$ & $-5.616 \mathrm{t}$ & $-2.016 \mathrm{t}$ & 4 \\
\hline $7 / 16$ & $1 / 16$ & 7 & $-7.658 \mathrm{t}$ & $-5.562 \mathrm{t}$ & $-2.096 \mathrm{t}$ & 2 \\
\hline $7 / 32$ & $7 / 16$ & $1^{*}$ & $-14.353 \mathrm{t}$ & $-14.713 \mathrm{t}$ & $0.360 \mathrm{t}$ & 1 \\
\hline $1 / 8$ & $1 / 4$ & $1^{*}$ & $-10.834 \mathrm{t}$ & $-10.917 \mathrm{t}$ & $0.083 \mathrm{t}$ & 1 \\
\hline $1 / 8$ & $7 / 16$ & $4 / 7^{*}$ & $-9.467 \mathrm{t}$ & $-9.566 \mathrm{t}$ & $0.098 \mathrm{t}$ & 4 \\
\hline $1 / 16$ & $5 / 16$ & $2 / 5^{*}$ & $-5.253 \mathrm{t}$ & $-5.274 \mathrm{t}$ & $0.021 \mathrm{t}$ & 6 \\
\hline $1 / 16$ & $7 / 16$ & $2 / 7^{*}$ & $-5.176 \mathrm{t}$ & $-5.197 \mathrm{t}$ & $0.022 \mathrm{t}$ & 2 \\
\hline
\end{tabular}

Table 4.2: Table of the energies and Chern numbers for the self-consistent solutions obtained in RMFT. $E_{0}=E_{\text {kin }}+E_{\text {pot }}$ represents the energy per $4 \times 4$ sublattice. The last column is the Chern number given by summing up the contribution from all the filled (mean-field) bands. The last five rows noted by an asterisk represent the fully polarized states for which $\nu^{*}=2 \nu$ is listed instead of $\nu$.

and third lines of Table 4.1). Staggered current (SC) patterns can also appear without CDW/BDW orders as described later on (see fourth line of Table 4.1).

Let us first examine the case $\Phi=\rho$. The results obtained for $J=0.3 t(t=1)$ show a homogeneous state and the RMFT band structure reveals a large band gap at the chemical potential. This corresponds to a mean-field (unprojected) state where the first Landau level is exactly filled. In the time-reversal symmetry broken state we may calculate the current for each bond as $J_{i j}=g_{i j \uparrow}^{t} \operatorname{Im}\left(\chi_{i j \uparrow} e^{i \phi_{i j}}\right)+g_{i j \downarrow}^{t} \operatorname{Im}\left(\chi_{i j \downarrow} e^{i \phi_{i j}}\right)$ while the charge hopping is $g_{i j \uparrow}^{t} \operatorname{Re}\left(\chi_{i j \uparrow} e^{i \phi_{i j}}\right)+g_{i j \downarrow}^{t} \operatorname{Re}\left(\chi_{i j \downarrow} e^{i \phi_{i j}}\right)$, where $\chi_{i j \sigma}=\left\langle c_{i \sigma}^{\dagger} c_{j \sigma}\right\rangle$. (The values of $\phi_{i j}$ at each bond for different $\Phi$ are shown in the Fig. 6.1.) For $\Phi=\rho$ all the bonds have zero current, confirming the homogeneous character of this state within the mean-field approach. The energy difference between RMFT and ED(Tables 4.2 and 4.3) is mainly due to the magnetic energy, that of RMFT being smaller than the ED, which also agrees with previous results [48].

It has been shown previously that, at fixed doping level $\delta=1 / 8$, the CFP exhibits an absolute minimum of the magnetic energy at $\Phi=7 / 16$ corresponding to the exact condition $\Phi=\rho$. However, after adding the competing kinetic energy, the total energy 
(a)

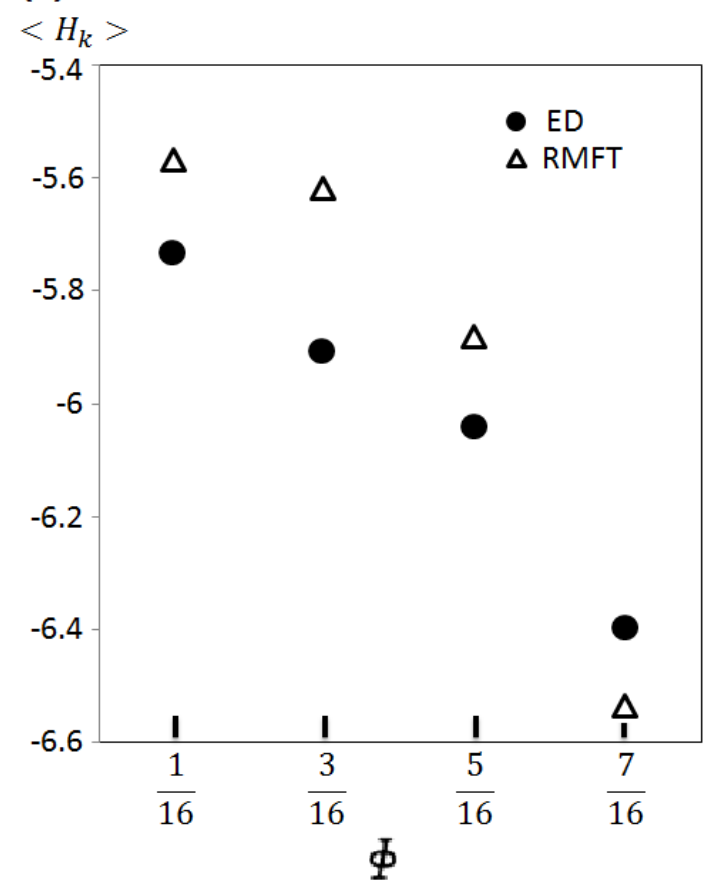

(b)

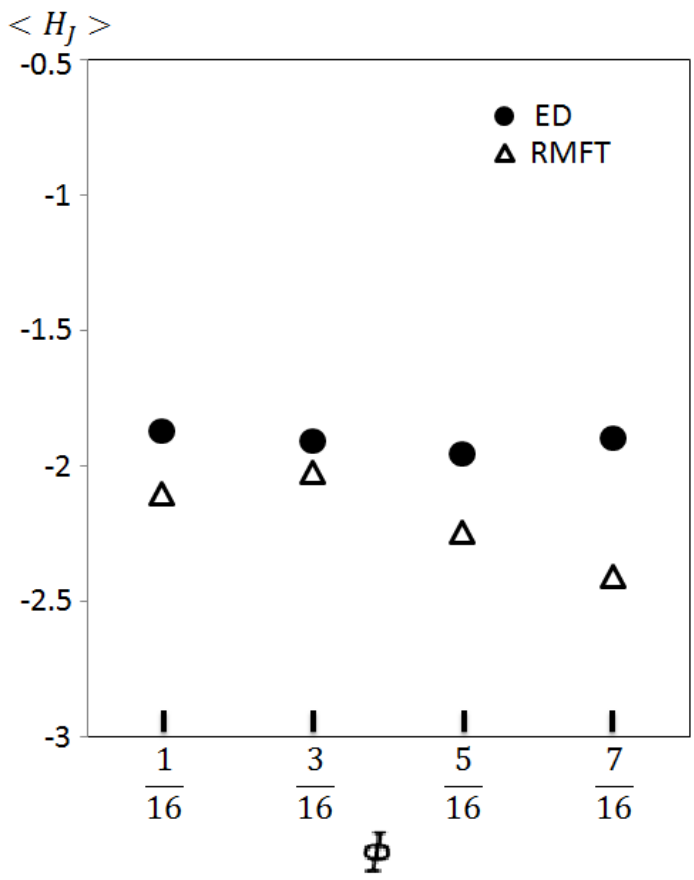

Figure 4.2: Comparison between RMFT and ED energies (per magnetic $4 \times 4$ unit cell). (a) Kinetic energy and (b) magnetic (potential) energy vs inserted flux $\Phi$. The doping level is fixed to $\delta=1 / 8$ and $J=0.3 t$. The numerical values are given in the Table 4.2.

was found to be lower for a smaller commensurate flux, at least at intermediate values of $J / t$ [117]. However, in Ref. [117] a simple $t-J$ Hamiltonian with $n o$ applied flux was considered, the flux entering only at the level of the projected CFP ansatz. Also, Ref. [117] did not take into account the possibility of CDW/BDW instabilities as well as the more sophisticated form of the Gutzwiller renormalization factors, both of which we have included here. When changing the inserted flux to $\Phi=\frac{5}{16}, \frac{3}{16}$, and $\frac{1}{16}$, the difference of the RMFT and ED magnetic energies becomes smaller as can be seen in Fig. 4.2. In contrast to Ref. [117], where the minimum of the kinetic energy was found at $\phi=\frac{1}{16}$, we find here with RMFT that it occurs at $\phi=\frac{7}{16}$, as for the magnetic part. This leads to a robust minimum of the total energy vs. flux profile and also generalizes to the case of the Affleck-Marston phase for which the minimal energy is found at $\Phi=\rho=1 / 2$.

Notably, for $\Phi=\frac{1}{16}$ and the same doping $\delta=1 / 8, \nu$ is equal to 7 which is also an integer, signifying that the first 7 Landau levels (of the mean-field spectrum) are filled. For this case, the real space pattern revealed by RMFT shows a staggered flux state with homogeneous current on each bond, that is, the current circulation directions are opposite 


\begin{tabular}{|c|c|c|c|c|c|c|c|}
\hline$\rho$ & $\Phi$ & $\nu / \nu^{*}$ & $S$ & $E_{0}$ & $E_{\text {kin }}$ & $E_{\text {pot }}$ & $C_{\mathrm{ED}}$ \\
\hline \hline $7 / 16$ & $7 / 16$ & 1 & 0 & -8.2901 & -6.39644 & -1.89369 & 2 \\
\hline $7 / 16$ & $5 / 16$ & $7 / 5$ & 0 & -8.0058 & -6.04586 & -1.95997 & 6 \\
\hline $7 / 16$ & $3 / 16$ & $7 / 3$ & 0 & -7.8204 & -5.90818 & -1.91226 & 6 \\
\hline $7 / 16$ & $1 / 16$ & 7 & 0 & -7.6298 & -5.73802 & -1.89179 & 14 \\
\hline $7 / 32$ & $7 / 16$ & $1^{*}$ & $7 / 2$ & -14.3874 & -14.7165 & 0.329042 & 1 \\
\hline $1 / 8$ & $1 / 4$ & $1^{*}$ & 2 & -11.2393 & -11.3132 & 0.0739077 & 1 \\
\hline $1 / 8$ & $7 / 16$ & $4 / 7^{*}$ & 2 & -9.4670 & -9.55201 & 0.0849988 & 4 \\
\hline $1 / 16$ & $5 / 16$ & $2 / 5^{*}$ & 1 & -5.2519 & -5.26527 & 0.0133967 & 6 \\
\hline $1 / 16$ & $7 / 16$ & $2 / 7^{*}$ & 1 & -5.1794 & -5.19852 & 0.0190752 & 2 \\
\hline
\end{tabular}

Table 4.3: Summary of the Lanczos exact diagonalization results.

between neighboring plaquettes. The reason is that again an integer number of Landau levels has been filled and the large band gap excludes the possibility of inhomogeneous modulation. Hence, it becomes clear that, for integral $\nu$, the band gap is large enough to suppress the lattice instability. The integer $\nu$ states are then adiabatically connected to band insulators, and we believe this scenario is generic beyond the two cases we have tested here.

Using similar arguments, we may already expect that for $\nu=7 / 5$ and $\nu=7 / 3$ lattice instabilities occur, since now the (mean-field) Landau levels are filled fractionally. Indeed we find them numerically, but they are of two different types. For $\nu=7 / 5$, we obtain two different self-consistent patterns (depending on the initial condition of the RMFT) with small but non-negligible energy difference and we concentrate on the one with lower energy first. As shown in Fig. 4.3, remarkably, the $\nu=7 / 5$ state does not exhibit charge modulation and has a uniform current amplitude on all bonds. However, the current pattern displays a $2 \times 2$ plaquette modulation, with two plaquettes carrying opposite current loops and two plaquettes with zero current circulation. This is also correlated with a $2 \times 2$ modulation of the hopping $\chi_{i j \sigma}$. In contrast, the RMFT solution with higher energy (corresponding to a local minimum in the variational space) bears a more complicated bond structure. For $\nu=7 / 3$, CDW order along with BDW order always develops as shown in Fig. 4.3. Interestingly, both cases can also be solved by including a non-zero pairing order parameter, indicating that either the Fermi level crosses bands instead of lying in a gap, or 


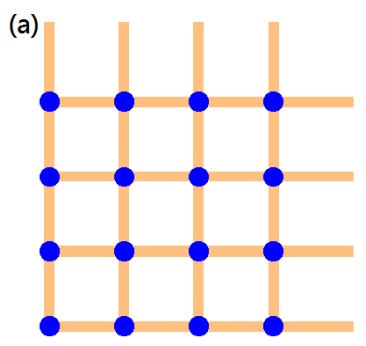

$\Phi=7 / 16$

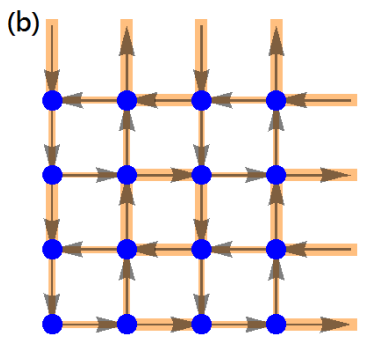

$\Phi=5 / 16$

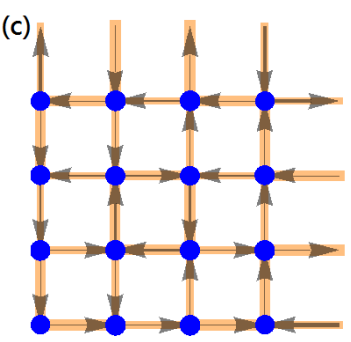

$\Phi=3 / 16$

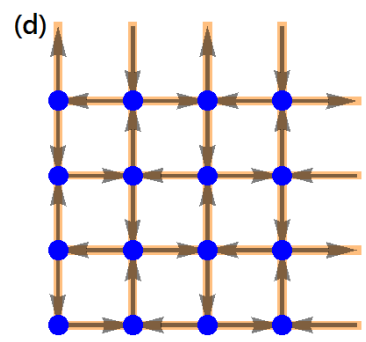

$\Phi=1 / 16$

Figure 4.3: Schematic patterns and results for the states in this subsection. (a)-(d) show the current and hopping patterns of each state within the $4 \times 4$ sublattice. The widths of the underlying orange bars and black arrows represent the magnitudes of hopping and current on each bond separately. The flows of current are indicated by the arrow directions. The numerical values are shown in Fig. 6.1.

the gap is rather small compared to the cases of $\nu=1$ or $\nu=7$. Hence, superconductivity appears, as has been discussed before [48], coexisting with bond and/or charge orders.

Note that to find translation symmetry breaking states in the model, ED cannot be used since in our case its applicability is limited to a $4 \times 4$ cluster. For such a small system, finite size effects destroy the translational invariance even of non-interacting magnetic models. This is due to the gauge choice we have to make in order to implement a magnetic flux $\Phi=q / 16, q=0, \cdots, 15$, which necessarily breaks the translational invariance within a $4 \times 4$ cluster. Of course, gauge invariance requires the full model to be translationally invariant. In the single particle picture, this can be accomplished by including degenerate states at nonzero momenta into the consideration. However, for the many-body system we are interested in, the system size accessible to ED is too small for these finite momentum single-particle states to contribute to the available Fock space. It is also not possible to effectively increase the system size by twisted boundary conditions as in the non-interacting case since this only ever allows us to reach a subset of all possible many-particle momenta: there are always many-particle momenta which correspond to different particles lying in different sectors of inserted flux, but twisted boundary conditions imply the same twisted flux for all particles. These shortcomings of ED render the comparison of charge, hopping and current density expectation values with RMFT difficult. 


\subsection{Fully polarized electron systems}

In the previous section we have considered a fixed doping of the $\rho=1 / 2$ Mott (AF) phase and studied how states evolve with changes in flux. In this section we will now vary the electron density while setting $\Phi$ to be $7 / 16$ or $5 / 16$. The remarkable phenomenon discussed here is the instability towards a fully polarized ferromagnet where all electronic spins are aligned in the same direction. This instability is driven by a gain of kinetic energy happening in the ferromagnetic state which supersedes the loss of magnetic energy when the electron density is small enough. We have indeed found that the energies of fully polarized states are lower than those of the singlets, both in RMFT and ED, for a number of cases, and we shall focus on those in this section.

For $\Phi=7 / 16$, we have studied several doping levels. For the cases we have considered, we found that the energies as calculated by RMFT or ED are very close(Tables 4.2 and 4.3 ) and the states we have found by either method are quite similar. This is not surprising since in fully polarized systems double occupancy is excluded by fermionic statistics, so that the projection operator $P_{\mathrm{G}}$ is no longer needed. Therefore, the Hamiltonian maps to a spinless electron system with nearest-neighbor repulsion. In this case, the RMFT renormalization factors become 1 as expected. Note that this is obtained only if the variational parameters of the nearest neighbor sites are included in the expression of the renormalization factors [40, 41, 45, 46] (small deviations from 1 occur nevertheless for $g_{i j}^{s, z}$ ). The agreement between RMFT and ED asserts the reliability of RMFT in the low-electron density regime, far away from the widely investigated low-doping regime. To further confirm this, we have also made the comparison for the case of $\rho=1 / 16$ and $\Phi=5 / 16$ and the energies from both side still agree remarkably well. All states we have obtained possess only very small currents, meaning that the phases of $\chi_{i j}$ tend to screen the phases from the applied magnetic flux in order to lower the kinetic energy. However, for $\rho=1 / 16$ and $\Phi=5 / 16$, there also emerge CDW and BDW orders which are not seen for $\Phi=7 / 16$. This follows from the differences in the respective non-interacting band structures. In Fig. 4.4(a) for $\rho=1 / 16$ and $\Phi=7 / 16\left(\nu^{*}=2 \nu=2 / 7\right)$, the Fermi level is located inside a large band gap between the second and the third (mean-field) 

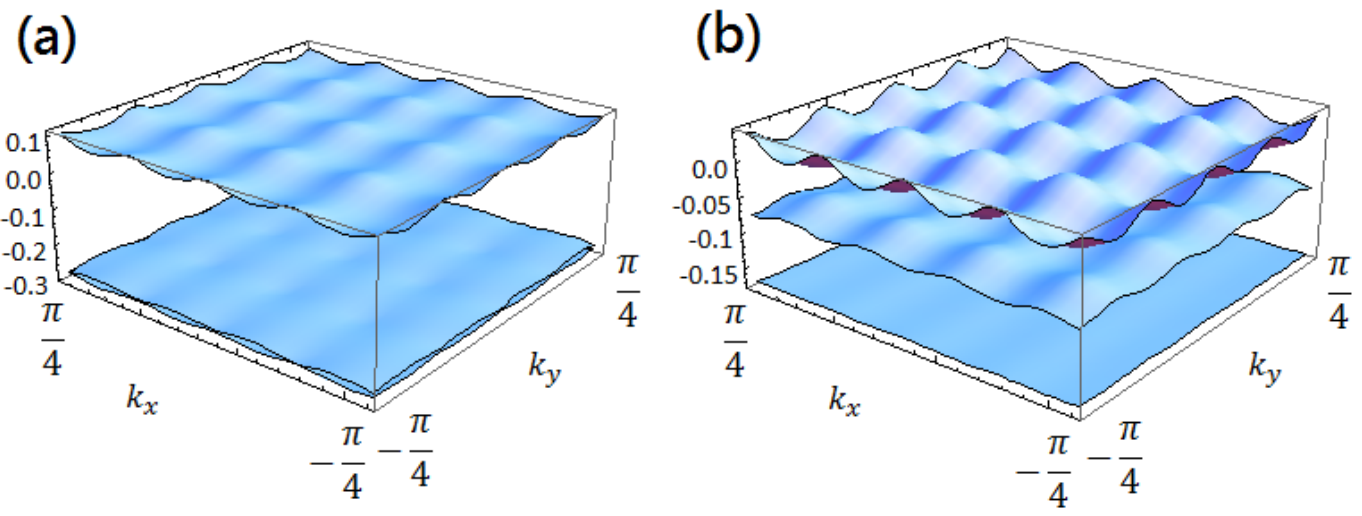

Figure 4.4: Band structure for the three lowest energy bands for (a) $\nu^{*}=2 / 7$ and (b) $\nu^{*}=2 / 5$. At this doping, the first two bands are filled. Note that in (a) the first two bands are almost degenerate.

band, producing a completely insulating state. In contrast, in Fig. 4.4(b) for $\rho=1 / 16$ and $\Phi=5 / 16\left(\nu^{*}=2 \nu=2 / 5\right)$, the band gap is much smaller (for the $k$ points where the two consecutive bands are closest, the gap value is around $0.03 t$ ), which allows for the instabilities that have been observed in our calculation.

\subsection{Topological properties}

Together with charge/bond ordering, it is also particularly interesting to look for the emergence of FQH-type states with topological order. At half-filling $(\rho=1 / 2)$ topological chiral spin liquids have been constructed as Gutzwiller projections of (non-interacting) wavefunctions with a completely filled band of Chern number $\pm 1[141,142,143]$. A related construction of topologically ordered states may also apply away from half-filling, at low doping and/or low electron density, and may be captured by the RMFT treatment of the Gutzwiller projector. In that case, our approach could point to situations where it may be energetically favorable for the system to accommodate a topologically ordered ground state.

Our first conclusion is that the $\nu=1$ and $\nu=7$ states in the integer quantum Hall regime are so robustly gapped that it is unlikely that further instabilities towards topologically ordered phases appear. What is left are the fully polarized uniform states with 


\begin{tabular}{|c|c|c|c|c|c|c|}
\hline$\rho$ & $\Phi$ & $\nu / \nu^{*}$ & $S$ & $C_{\mathrm{ni}}$ & $C_{\mathrm{RMFT}}$ & $C_{\mathrm{ED}}$ \\
\hline \hline $7 / 16$ & $7 / 16$ & 1 & 0 & 2 & 2 & 2 \\
\hline $7 / 16$ & $5 / 16$ & $7 / 5$ & 0 & 10 & 2 & 6 \\
\hline $7 / 16$ & $3 / 16$ & $7 / 3$ & 0 & 6 & 4 & 6 \\
\hline $7 / 16$ & $1 / 16$ & 7 & 0 & 14 & 2 & 14 \\
\hline $7 / 32$ & $7 / 16$ & $1^{*}$ & $F P$ & 1 & 1 & 1 \\
\hline $1 / 8$ & $1 / 4$ & $1^{*}$ & $F P$ & 1 & 1 & 1 \\
\hline $1 / 8$ & $7 / 16$ & $4 / 7^{*}$ & $F P$ & 4 & 4 & 4 \\
\hline $1 / 16$ & $5 / 16$ & $2 / 5^{*}$ & $F P$ & 6 & 6 & 6 \\
\hline $1 / 16$ & $7 / 16$ & $2 / 7^{*}$ & $F P$ & 2 & 2 & 2 \\
\hline
\end{tabular}

Table 4.4: Table comparing the Chern numbers obtained in the non-interacting case, in the (non-superconducting) RMFT self-consistent solutions and by Lanczos ED. In the two first cases, the Chern numbers are given by summing up the contribution from all the filled bands. The last five rows noted by an asterisk represent the fully polarized states for which $\nu^{*}=2 \nu$ is listed instead of $\nu$.

$\Phi=7 / 16$. The simplest prerequisite for the numerical realization of a FQH state in a system with periodic boundary conditions (i.e., a two-torus) is a topological ground state degeneracy(GSD) [144]. In a given symmetry sector we expect nearly degenerate states which are separated by a gap from all other states(If a system realizes a bosonic $\nu=1 / 2$ Laughlin state, this topological degeneracy should be two, for example.). Figure A.2 in the Appendix A shows the ED energy spectra for each case that we have discussed, resolved into $S_{z}$ subspaces. We can see that there is no GSD even though for certain $S_{z}$ the first two energy levels are fairly close. For example, for $\nu=2 / 7$ the $S_{z}=0$ sector has two nearly degenerate states at low energy, but one has $S=0$ and the other one has $S=2$. Therefore, these states cannot be topologically degenerate partners. Moreover, we checked that the manifold spanned by these two states has even Chern number and thus cannot realize a FQH state.

The reason why it is hard for fully polarized phases to realize a FQH state in the model we study is as follows: The dominant Hubbard interaction term is very local. In the FQH effect, interaction terms, projected into the single particle states of a given Landau level, are expanded in Haldane pseudopotentials. An ultralocal interaction contributes to the $V_{0}$ 
pseudopotential, which gives rise to the bosonic Laughlin state. For the simplest fermionic FQH Laughlin state, the longer-ranged pseudopotential $V_{1}$ is required. However, as has been studied in the context of fractional Chern insulators [145], the ultra-local Hubbard interaction translates into a dominant $V_{0}$ component after projection into a given band with nonvanishing Chern number.

Although directly observing FQH states in our calculations seems therefore unlikely, the states we have obtained still have (generically) interesting topological features associated to non-zero integer Chern numbers [146] and Hall conductance given by

$$
\sigma=C \frac{e^{2}}{h}
$$

with $C$ being the (many-body) Chern number, and the Planck constant $h$ and the electronic charge $e$ have been re-introduced for clarity. For RMFT, the way of calculating Chern numbers is to integrate the Berry curvature of each mean-field band as has been shown in Ref. [123]. In ED the many-body Chern numbers [147] are computed by introducing twisted boundary conditions [148, 149] (see Appendix A for details). The Chern numbers obtained by ED and RMFT (for the non-superconducting solutions) are compared with each other and also with the non-interacting case in Table 4.4. We note that at low enough electron filling, i.e., below 1/4-filling, all Chern numbers agree with the non-interacting ones (provided one assumes a ferromagnetic state, e.g., considers spinless fermions) showing that the effect of the interaction is moderate in this regime. In particular we observe that the lattice instabilities found in RMFT do not affect the topological character of the states. In contrast, discrepancies appear when approaching the Mott insulating phase, in the low doping regime at $\rho=7 / 16$. This signals that interactions play a crucial role there and obtaining the correct many-body Chern numbers of these correlated states is tedious: on one hand, the approximate way of treating the Gutzwiller projection in RMFT may not capture correctly the topological properties and/or, on the other hand, finite size effects in ED may also lead to deviations. It is, however, likely that Chern numbers close to the Mott insulating phase are different from those of the non-interacting case. A noticeable counterexample is the case $\rho=\Phi=7 / 16, \nu=1$ where the Chern number 
$C=2$ obtained by ED and RMFT agrees with the non-interacting limit. This suggests an adiabatic continuity from the non-interacting to the interacting case, which we have explicitly checked to hold in ED using a Hofstadter-Hubbard model where we increased the interaction strength $U$ gradually. 


\section{Chapter 5}

\section{Conclusions and Outlooks}

The results reported above are all based upon the well-established renormalized mean-field theory [31] and GWA [56] for a well-studied $t-J$ or $t-t^{\prime}-J$ model. Although they do not provide extremely accurate numbers, as many sophisticated numerical methods do, our results show that they do capture the most important physics of the strong correlation. For understanding the cuprate, first of all, this strong correlation provides a site-dependent Gutzwiller renormalization that produces many exotic solutions of PDW stripes and/or CBs intertwined with modulations of charge density and/or spin density. These results show quantitative agreement with some of the key experiments [15, 24, 25]. Because site-renormalization is extremely local, the effect of the Fermi surface or wave vectors $k_{F}$ is absent. Our model does not require the second or third neighbor hopping to provide a Fermi surface with nesting vectors or "hot spots" [33, 51, 91]. Thus, in our theory, there are no unique wave vectors for the charge density waves or CBs. Although we have mainly focused on the structures with a period of $4 a_{0}$ so far, our preliminary study also finds charge-ordered states with periods of $5 a_{0}$ and even $3 a_{0}$. States with a longer period should be possible, and they could also have degenerate energies [46, 54]. If we allow a pattern with multiple periods, nPDW can be formed and we could have states with fractional or incommensurate periods.

An important consequence of having all these charge-ordered states originating from the same Hamiltonian and physics is that these states are not the usual "competing states" we are familiar with. They do not stay in a deep local minimum in the energy landscape. 
They are actually quite fragile and can easily evolve into each other, as we have already demonstrated with the nPDW stripe, which evolved from a mixture of AP-CDW and an uniform d-SC state. Other examples of the mixture of stripes listed in Table 3.1 can be easily constructed. For real cuprates, there are many other interactions in addition to our $t$ and $J$ that will alter the preferences of these states. For example, a weak electron lattice interaction could make the IP-CDW-SDW stripe much more stable against the dSC-AFM state [42]. Including special Fermi surface features could also enhance CDW for certain periods. However, none of these interactions are as important and necessary as the site renormalization due to strong Mott physics to produce these charge-ordered states.

With second nearest hopping $t^{\prime}$, we have shown that there exist low-energy, commensurate and incommensurate charge modulated renormalizedmean field solutions of the $t-t^{\prime}-J$ model that are not the ground state at any filling, but still which are extremely close to the energy of the uniform superconducting state. Furthermore, the nPDW are intertwined with modulated superconductivity, and display properties remarkably similar to STM observations of the 1D modulated states seen on the surface of BSCCO and NaCCOC. These are well-established features of cuprate physics that have intrigued workers in the field for almost a decade, but until now have defied explanation. Among these properties are the same spectra and pattern of tunneling conductance maps within the unit cell as observed by STM on under- to optimally doped BSCCO and NaCCOC. To calculate these patterns, as well as continuum LDOS spectra within the unit cell, we employed the new Wannier function-based method of Ref. [119], which enables the calculation of the wavefunctions in the correlated state at any $3 \mathrm{D}$ position, including several angstrom above the surface where the STM tip is placed. This gives us an unprecedented ability to compare with details of the experiments in the charge ordered regime.

In addition, the bias dependence of intra-unit cell $d-, s^{\prime}-$ and $s$-form factors and their spatial phase difference were obtained in the nPDW state and display good agreement with the STM observations. The energy of the peak $d$-wave form factor depends on doping in a manner similar to the pseudogap. Note that with the exception of us, previous theories of charge ordered states in $t-t^{\prime}-J$ type models treated only commensurate $\left(4 a_{0}\right)$ 
charge order states, and could express observables only in terms of bias-independent bond variables.

One of the surprises we found is that without adjustable parameters in our calculations, we are able to get many quantities very close to experimental values and also have very good agreement with very sophisticated numerical works that go much beyond mean-field theory. Considering we are doing a mean-field calculation, this is even more surprising. One main reason could be that the GWA is really effective in catching the main physics of the $t-J$ model. Based on this premise, we can now provide a very simple picture about the cuprate phase diagrams. Starting at half-filling, the model has the RVB proposed by Anderson [12] dominate in the Mott insulator. RVB has the $d$-wave pairing and bond order intertwined. But without charge present both of them are actually the variational parameters or hidden orders we defined in Eq. 6.4. When holes are doped into the lattice, RVB tends to localize the charges to prohibit its fluctuation. Once the localization has failed possibly after the antiferromagnetism is destroyed by the dopant, the system starts to form these unidirectional PDW states which has charge density intertwined with RVB (pairing and bond order). These states have a gap in the antinodal region and in the nodal region a Fermi arc with only a fractional DOS survived. When there is too much doping that these density waves can no longer be viable, then we lose the Mott physics and recovered a FLS [150]. These states then develop an average uniform SC pairing order at lower temperatures although it is relatively small in comparison with large magnitude of pairing modulation. Of course, the phase fluctuation will become more important as temperature rises [36, 93] and mean field results will be revised.

The theory we propose depends on the presence of a PDW state in the PG phase. There is a way to test this hypothesis besides the possibility of using STS [1], which has to worry about the rapid pairing phase variation in a few lattice spacing and also the measurement being most likely at a higher temperature. For a PDW state in $x$-direction, the magnitude of the gap in the $y$-antinode $(0, \pi)$ is about the same [4] as the gap in the $x$-antinode $(\pi, 0)$ as shown in Fig. 3.11 and 3.12. This is contrary to what one would expect if we only have a pure CDW in the $x$-direction. Then the gap opening due to zone folding should be larger 
in the folding direction. The $x$ and $y$ asymmetry of the Fermi arc, shown in Fig. 3.15(f), may be used to distinguish the arc from part of the Fermi pocket [80, 81]. We can also examine the particle-hole asymmetry in the PG phase. IPDW will have very similar result as the nPDW state measured by ARPES [82]. Particle-hole asymmetry should be observed away from the Fermi arc. This could be a sign for the presence of finite momentum Cooper pairs [39].

In particular, we discussed the possibility that impurities stabilize the charge order, leading to the disordered 1D patterns observed in STM on BSCCO and NaCCOC. This disordered ground state would also be consistent with the short-range charge-order observed by resonant $x$-ray scattering [68]. In such a system, a magnetic field should suppress superconductivity and eventually favor long-range charge order, as observed in experiments. It has been shown that a magnetic field about $10 T$ is enough to induce a long-range ordered CDW or PDW [127, 139]. Since $10 T$ is quite small, it may be possible to generate the long-range order by having a thin tetragonal single layer cuprate deposited on a strained substrate. We have looked at the case with the hopping rate in the $x$ direction $t_{x}$ less than $t_{y}$ in the $y$ direction. The preliminary result shows that the energy of nPDW state for doping concentration $\delta=0.08$ is now lower than the uniform $d$-wave SC state if $t_{x}<0.84$ $t_{y}$. This is consistent with previous work on stripe states at $x=0.125$ [40]. But here we probably overestimate the strength of the uniform state. In real material a small difference between $t_{x}$ and $t_{y}$ might be enough to stabilize an IPDW/nPDW. Since the $x$-directional nPDW has a much lower energy than the $y$-directional nPDW, the system is likely to be dominated by only $x$-directional nPDW, and a unidirectional IPDW at $T>T_{c}$. It may also be possible to have a phase coherent IPDW state in a small temperature window that will be a truly new phase. Even without invoking $t_{x}<t_{y}$, as shown in Fig. 3.11 and 3.12, the spectra near $(\pi, 0)$ and $(0, \pi)$ are very different. Now with $t_{x}<t_{y}$, the UPOP has the $s^{\prime}+d$ symmetry with pairing in $x$ direction larger than in $y$. On the other hand, the energy gap near the $x$-direction antinode $(\pi, 0)$ is getting smaller as strain increases, while the gap near $(0, \pi)$ becomes larger. Thus IPDW in the PG phase could be detected with ARPES in this system. 
Moreover, motivated by recent experimental and numerical developments, we studied the Harper-Hofstadter model in the presence of strong correlations, which corresponds to the $t-J$ model in an orbital magnetic field. By employing a RMFT approach, supplemented by Lanczos ED calculations, we endeavored to find novel condensed matter phases for fermionic systems. In particular, we have focused on CFPs and several ferromagnetic phases. Although we failed to observe topologically ordered states, neither of singlet character nor fully polarized, topologically non-trivial states with non-zero Chern numbers have been identified in the presence of interaction. We found CFPs which realize an integer quantum Hall system. Those at fractional filling fraction $\nu$ generically exhibit lattice instabilities. For fully polarized states, occurring at low electron filling, RMFT and ED agree precisely with each other with regard to the GS energies and Chern numbers. Moreover, we showed that the effect of a staggered potential on destabilizing the topological state depends weakly on the interaction, which is demonstrated in Appendix B. Note that, close to the Mott insulating phase, i.e., at low (hole) doping, RMFT and ED results for the Chern numbers disagree with each other, revealing strong interaction effects that render the computation of the topological properties of the states difficult. Therefore, it is interesting to realize the system we propose in experimental setups. It has been shown that it is possible to investigate the Fermi-Hubbard model with degenerate Fermi gases with atomic species such as ${ }^{6} \operatorname{Li}(37,38)$, manipulated within optical lattices [151]. In order to include(synthetic) gauge fields, laser assistant tunneling can be applied with two laser beams controlling the hopping of nearby sites with an additional flux phase [97, 98]. We suggest a combination of these techniques for an experimental investigation of our system. Compared with the experimental setup of the Harper-Hofstadter Hamiltonian with interaction, however, the agreement between our results and those from the cold atom experiment suggests that the $t-J$ Hamiltonian is relevant for describing the physics of interacting fermions under external magnetic flux. Our results give a taste of the phenomena emerging from the strongly correlated Hofstadter Hamiltonian and motivate further experimental and theoretical studies. 


\section{Chapter 6}

\section{Sommaire}

Cette thèse vise à utiliser le $t-J$ Hamiltonian de la corrélation forte pour mieux comprendre la micro-fonctionnalité des scénarios de matériau condensé. Quand on aborde ces modèles, il est important de les exploiter de façon numérique. Dans cette thése, nous utiliserons la manière qui s'appelle "Renormalized Mean-Field Theory"(RMFT) pour le $t-J$ Hamiltonian. Grâce à $\mathrm{M}$. Gutzwiller, ce que nous devons faire est simplement de chiffrer des paramètres qui incluent l'influence de la corrélation électronique et de les mettre avant chaque partie du Hamiltonian. Après ce calcul, nous calculerons l'Hamiltonian du champ moyen de manière standard. Ceci sera notre façon principale pour aborder des questions physiques.

\subsection{Méthode}

Je vais discuter tout d'abord cette méthode en détail à partir de l'Hamiltonian de $t-J$. Je vais aussi montrer comment nous pouvons calculer des charactéristiques importantes comme LDOS et spectres avec Bogoliubov-deGenne fonction de sauvegarde.

\subsection{1 Équation BdG de Hamiltonian du champ moyen}

Dans cette thése, nous considons le modèle de $t-J$ en 2D, c'est-à-dire dans la limite que $U$ du modèle Hubbard est extrêmement grand. Par fois nous ajoutons un champ extérieur 
de la magnétique.

$$
\begin{gathered}
H=\underbrace{-\sum_{\langle i, j\rangle, \mu} P_{\mathrm{G}}\left(t_{i j} c_{i \mu}^{\dagger} c_{j \mu}+\text { h.c. }\right) P_{\mathrm{G}}}_{H_{\mathrm{kin}}} \underbrace{\sum_{\langle i, j\rangle} J \boldsymbol{S}_{i} \cdot \boldsymbol{S}_{j}}_{H_{\mathrm{pot}}}, \\
t_{i j}=t e^{\mathrm{i} A_{i j}}=t_{j i}^{*}, \quad \boldsymbol{S}_{i}=\sum_{\mu, \nu} c_{i \mu}^{\dagger} \boldsymbol{\sigma}_{\mu \nu} c_{j \nu},
\end{gathered}
$$

$c_{i \mu}^{\dagger}\left(c_{i \mu}\right)$ est l'opérateur de la création(anéantissement) pour un électron avec du spin $\mu=\uparrow$ ,$\downarrow$ sur le site du treillis $i . n_{i \mu}=c_{i \mu}^{\dagger} c_{i \mu}$ est l'opérateur par spin du site. $P_{\mathrm{G}}=\prod_{i}\left(1-n_{i \uparrow} n_{i \downarrow}\right)$ est l'opérator de Gutzwiller qui fonctionne comme l'interdiction d'occupation de deux électrons au même temps. $\boldsymbol{\sigma}=\left(\sigma_{x}, \sigma_{y}, \sigma_{z}\right)^{\mathrm{T}}$ est le vecteur de $2 \times 2$ metrice de Pauli. Dans la transformation précis du modèle Hubbard, il y a une terme de saut triple, qui est à l'unité de $t^{2} / U$. Cette terme n'influence pas beaucoup le résutat et donc nous n'en soucions pas. $J$ est égal de $0.3 t$ tout cette thése.

Le champ de magnétique entre l'Hamiltonian dans la forme de la phase $A_{i j}=\int_{i}^{j} \boldsymbol{A}(\boldsymbol{x})$. $d \boldsymbol{x}$, où le potential de vecteur $\boldsymbol{A}(\boldsymbol{x})$ est défini par $\boldsymbol{B}(\boldsymbol{x})=\nabla \times \boldsymbol{A}(\boldsymbol{x})$. Le flux per plaquette est comme $F=\int_{\square} \boldsymbol{B}(\boldsymbol{x}) \cdot d \boldsymbol{\Sigma}=A_{i, i+\hat{x}}+A_{i+\hat{x}, i+\hat{x}+\hat{y}}+A_{i+\hat{x}+\hat{y}, i+\hat{y}}+A_{i+\hat{y}, i}$. Ici nous choissisons que $F=2 \pi \Phi$, with $\Phi$ avec $\Phi$ est égal de $\frac{7}{16}$, $\frac{5}{16}$, etc. $A_{i j}=0$ quand nous luttons le problème de cuprate.

Le procédé standard de RMFT est d'abord à remplacer l'opérateur Gutzwiller par des facteurs renormalisé $g^{t}$ and $g^{s}$ et donc:

$$
\begin{aligned}
& \left\langle\Psi\left|c_{i \mu}^{\dagger} c_{j \mu}\right| \Psi\right\rangle=g_{i j \mu}^{t}\left\langle\Psi_{0}\left|c_{i \mu}^{\dagger} c_{j \mu}\right| \Psi_{0}\right\rangle, \\
& \left\langle\Psi\left|\boldsymbol{S}_{i} \cdot \boldsymbol{S}_{j}\right| \Psi\right\rangle=g_{i j}^{s}\left\langle\Psi_{0}\left|\boldsymbol{S}_{i} \cdot \boldsymbol{S}_{j}\right| \Psi_{0}\right\rangle
\end{aligned}
$$

où $\left|\Psi_{0}\right\rangle$ est la fonction sans projection et $|\Psi\rangle=P_{\mathrm{G}}\left|\Psi_{0}\right\rangle$. Alors, cette Hamiltonian devient:

$$
\begin{aligned}
H= & -\sum_{\langle i, j\rangle \mu} g_{i j \mu}^{t} t_{i j} e^{i A_{i j}}\left(c_{i \mu}^{\dagger} c_{j \mu}+\text { h.c. }\right) \\
& +\sum_{\langle i, j\rangle} J\left[g_{i j}^{s, z} S_{i}^{s, z} S_{j}^{s, z}+g_{i j}^{s, x y}\left(\frac{S_{i}^{+} S_{j}^{-}+S_{i}^{-} S_{j}^{+}}{2}\right)\right]
\end{aligned}
$$


où $g_{i j \sigma}^{t}, g_{i j}^{s, z}$, and $g_{i j}^{s, x y}$ sont les facteurs de Gutzwiller, qui dépendent de valeurs de paramètres $\Delta_{i j \mu}^{v}, \chi_{i j \mu}^{v}, m_{i}^{v}$, et $\delta_{i}$ :

$$
\begin{aligned}
& m_{i}^{v}=\left\langle\Psi_{0}\left|S_{i}^{z}\right| \Psi_{0}\right\rangle \\
& \Delta_{i j \mu}^{v}=\mu\left\langle\Psi_{0}\left|c_{i \mu} c_{j \bar{\mu}}\right| \Psi_{0}\right\rangle \\
& \chi_{i j \mu}^{v}=\left\langle\Psi_{0}\left|c_{i \mu}^{\dagger} c_{j \mu}\right| \Psi_{0}\right\rangle \\
& \delta_{i}=1-\left\langle\Psi_{0}\left|n_{i}\right| \Psi_{0}\right\rangle
\end{aligned}
$$

où $\left|\Psi_{0}\right\rangle$ est la fonction sans projection. $v$ attaché à chaque paramètre représente que ces quantités sont variationnels au lieu de quantité réel. Pour la phase $\left(A_{i j}\right)$, nous suivons la définition de Ref. [117]. Le nombre pour flux par plaquette $\Phi$ est montré au Fig. 6.1. Nous considons des facteurs Gutzwiller proposé par Ogata et Himeda [41, 45]:

$$
\begin{aligned}
& g_{i j \mu}^{t}=g_{i \mu}^{t} g_{j \mu}^{t} \\
& g_{i \mu}^{t}=\sqrt{\frac{2 \delta_{i}\left(1-\delta_{i}\right)}{1-\delta_{i}^{2}+4\left(m_{i}^{v}\right)^{2}} \frac{1+\delta_{i}+\mu 2 m_{i}^{v}}{1+\delta_{i}-\mu 2 m_{i}^{v}}} \\
& g_{i j}^{s, x y}=g_{i}^{s, x y} g_{j}^{s, x y} \\
& g_{i}^{s, x y}=\frac{2\left(1-\delta_{i}\right)}{1-\delta_{i}^{2}+4\left(m_{i}^{v}\right)^{2}} \\
& g_{i j}^{s, z}=g_{i j}^{s, x y} \frac{2\left(\left(\bar{\Delta}_{i j}^{v}\right)^{2}+\left(\bar{\chi}_{i j}^{v}\right)^{2}\right)-4 m_{i}^{v} m_{j}^{v} X_{i j}^{2}}{2\left(\left(\bar{\Delta}_{i j}^{v}\right)^{2}+\left(\bar{\chi}_{i j}^{v}\right)^{2}\right)-4 m_{i}^{v} m_{j}^{v}} \\
& X_{i j}=1+\frac{12\left(1-\delta_{i}\right)\left(1-\delta_{j}\right)\left(\left(\bar{\Delta}_{i j}^{v}\right)^{2}+\left(\bar{\chi}_{i j}^{v}\right)^{2}\right)}{\sqrt{\left(1-\delta_{i}^{2}+4\left(m_{i}^{v}\right)^{2}\right)\left(1-\delta_{j}^{2}+4\left(m_{j}^{v}\right)^{2}\right)}}
\end{aligned}
$$

où $\bar{\Delta}_{i j}^{v}=\sum_{\mu} \Delta_{i j \mu}^{v} / 2$ and $\bar{\chi}_{i j}^{v}=\sum_{\mu} \chi_{i j \mu}^{v} / 2$. Dans la présence d'AF, $\Delta_{i j \uparrow}^{v} \neq \Delta_{i j \downarrow}^{v}$. Pour les états maillots $m_{i}^{v}$ est égal de zéro et $n_{i \uparrow}=n_{i \downarrow}=\frac{1}{2}\left(1-\delta_{i}\right)$. Cependant, pour les états complètement polarisé $m_{i}^{v}=n_{i \uparrow} / 2$ ainsi que $n_{i \uparrow}=\left(1-\delta_{i}\right), n_{i \downarrow}=0$, où nous laissons tous électrons spin en haut. Cette combination de facteur Gutzwiller est conformité avec le calcul maillot de Monde Carlo [41, 45].

Aprés que nous remplacons l'opérateur de projection par facteurs Gutzwiller défini au Eq. 6.4, l'energie d'Hamiltonian(Eq. 6.3) devient le suivant avec quatre parties et 

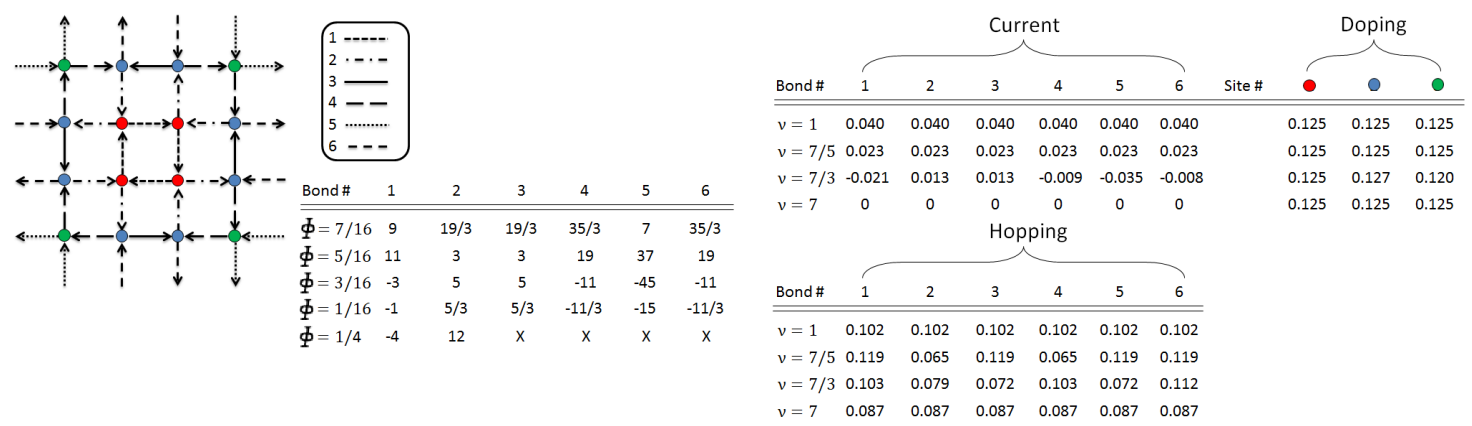

Figure 6.1: Distribution of the phases $\phi_{i j}$ on the bonds of $4 \times 4$ and $2 \times 2$ unit cells (on the 2-torus) for the flux densities $\Phi$ considered in this work(times $\pi / 32$ ). Arrows again indicate the directions of current and negative signs stand for opposite flows. The flux density $\Phi=1 / 4$ has only two different bonds (bond 1 and 2). The right panel shows detailed numbers of variables for the patterns we have obtained. Those patterns will be discussed later.

paramétres du champ moyen:

$$
\begin{aligned}
E=\left\langle\Psi_{0}|H| \Psi_{0}\right\rangle= & -\sum_{i, j, \mu} g_{i j \mu}^{t} t e^{i A_{i j}}\left(\chi_{i j \mu}^{v}+\text { h.c. }\right) \\
& -\sum_{\langle i, j\rangle \mu} J\left(\frac{g_{i j}^{s, z}}{4}+\frac{g_{i j}^{s, x y}}{2} \frac{\Delta_{i j \bar{\mu}}^{v *}}{\Delta_{i j \mu}^{v *}}\right) \Delta_{i j \mu}^{v *} \Delta_{i j \mu}^{v} \\
& -\sum_{\langle i, j\rangle \mu} J\left(\frac{g_{i j}^{s, z}}{4}+\frac{g_{i j}^{s, x y}}{2} \frac{\chi_{i j \bar{\mu}}^{v *}}{\chi_{i j \mu}^{v *}}\right) \chi_{i j \mu}^{v *} \chi_{i j \mu}^{v} \\
& +\sum_{\langle i, j\rangle} g_{i j}^{s, z} J m_{i}^{v} m_{j}^{v}
\end{aligned}
$$

Ensuite, nous voulons minimiser l'energie avec deux conditions: $\sum_{i} n_{i}=N_{\mathrm{e}}$ and $\left\langle\Psi_{0} \mid \Psi_{0}\right\rangle=1$. Par conséquent, notre fonction devient

$$
W=\left\langle\Psi_{0}|H| \Psi_{0}\right\rangle-\lambda\left(\left\langle\Psi_{0} \mid \Psi_{0}\right\rangle-1\right)-\epsilon\left(\sum_{i} n_{i}-N_{\mathrm{e}}\right)
$$

L'Hamiltonian du champ moyen est

$$
H_{\mathrm{MF}}=\sum_{\langle i, j\rangle \mu} \frac{\partial W}{\partial \chi_{i j \mu}^{v}} c_{i \mu}^{\dagger} c_{j \mu}+\text { h.c. }+\sum_{\langle i, j\rangle \mu} \frac{\partial W}{\partial \Delta_{i j \mu}^{v}} \mu c_{i \mu} c_{j \bar{\mu}}+\text { h.c. }+\sum_{i, \mu} \frac{\partial W}{\partial n_{i \mu}} n_{i \mu}
$$

Eq. (6.8) satisfait à l'équation Schrödinger $H_{\mathrm{MF}}\left|\Psi_{0}\right\rangle=\lambda\left|\Psi_{0}\right\rangle$. Les trois dérivés sont défini 
par:

$$
\begin{aligned}
H_{i j \mu}=\frac{\partial W}{\partial \chi_{i j \mu}^{v}}= & -J\left(\frac{g_{i j}^{s, z}}{4}+\frac{g_{i j}^{s, x y}}{2} \frac{\chi_{i j \mu}^{v *}}{\chi_{i j \mu}^{v *}}\right) \chi_{i j \mu}^{v *}-g_{i j \mu}^{t} t_{i j} e^{i A_{i j}}+\frac{\partial W}{\partial g_{i j}^{s, z}} \frac{\partial g_{i j}^{s, z}}{\partial \chi_{i j \mu}^{v}} \\
D_{i j}^{*}=\frac{\partial W}{\partial \Delta_{i j \uparrow}^{v}}= & -J\left(\frac{g_{i j}^{s, z}}{4}+\frac{g_{i j}^{s, x y}}{2} \frac{\Delta_{i j \downarrow}^{v *}}{\Delta_{i j \uparrow}^{v *}}\right) \Delta_{i j \uparrow}^{v *}+\frac{\partial W}{\partial g_{i j}^{s, z}} \frac{\partial g_{i j}^{s, z}}{\partial \Delta_{i j \uparrow}^{v}}
\end{aligned}
$$

et

$$
\epsilon_{i \mu}=-\frac{\partial W}{\partial n_{i \mu}}=\epsilon-\sum_{j} \frac{\partial W}{\partial g_{i j}^{s, x y}} \frac{\partial g_{i j}^{s, x y}}{\partial n_{i \mu}}-\sum_{j} \frac{\partial W}{\partial g_{i j}^{s, z}} \frac{\partial g_{i j}^{s, z}}{\partial n_{i \mu}}-\sum_{j \mu^{\prime}} \frac{\partial W}{\partial g_{i j \mu^{\prime}}^{t}} \frac{\partial g_{i j \mu^{\prime}}^{t}}{\partial n_{i \mu}}
$$

Eq. (6.10) est la potential de chimie locale. $H_{\mathrm{MF}}$ peut être récrit à la forme d'équation BdG.

$$
H_{\mathrm{MF}}=\left(c_{i \uparrow}^{\dagger}, c_{i \downarrow}\right)\left(\begin{array}{cc}
H_{i j \uparrow} & D_{i j} \\
D_{j i}^{*} & -H_{j i \downarrow}
\end{array}\right)\left(\begin{array}{c}
c_{j \uparrow} \\
c_{j \downarrow}^{\dagger}
\end{array}\right)
$$

Nous pouvons résoudre $H_{\mathrm{MF}}$ pour obtenir des valeurs propres. Le nombre positif est égal du nombre négatif avec ses vecteurs propres correspondants $\left(u_{i}^{n}, v_{i}^{n}\right)$. Avec ces vecteurs, nous pouvons calculer les paramètres d'ordre au zéro température par les équations suivantes:

$$
\begin{aligned}
& n_{i \uparrow}=\left\langle c_{i \uparrow}^{\dagger} c_{i \uparrow}\right\rangle=\sum_{n}\left|u_{i}^{n}\right|^{2} f\left(E_{n}\right) \\
& n_{i \downarrow}=\left\langle c_{i \downarrow}^{\dagger} c_{i \downarrow}\right\rangle=\sum_{n}\left|v_{i}^{n}\right|^{2}\left(1-f\left(E_{n}\right)\right) \\
& \Delta_{i j \uparrow}^{v}=\left\langle c_{i \uparrow} c_{j \downarrow}\right\rangle=\sum_{n_{+}} u_{i}^{n} v_{j}^{n *}\left(1-f\left(E_{n}\right)\right)-u_{j}^{n} v_{i}^{n *}\left(1-f\left(-E_{n}\right)\right) \\
& \Delta_{i j \downarrow}^{v}=-\left\langle c_{i \downarrow} c_{j \uparrow}\right\rangle=\sum_{n_{+}} u_{j}^{n} v_{i}^{n *}\left(1-f\left(E_{n}\right)\right)-u_{i}^{n} v_{j}^{n *}\left(1-f\left(-E_{n}\right)\right) \\
& \chi_{i j \uparrow}^{v}=\left\langle c_{i \uparrow}^{\dagger} c_{j \uparrow}\right\rangle=\sum_{n} u_{j}^{n} u_{i}^{n *} f\left(E_{n}\right) \\
& \chi_{i j \downarrow}^{v}=\left\langle c_{i \downarrow}^{\dagger} c_{j \downarrow}\right\rangle=\sum_{n} v_{i}^{n} v_{j}^{n *}\left(1-f\left(E_{n}\right)\right)
\end{aligned}
$$

La somme de $n_{+}$signifie seulement pour les vecteurs propres avec des énergies positives. 
$f\left(E_{n}\right)$ est la distribution de Fermi-Dirac:

$$
f\left(E_{n}\right)=\frac{1}{e^{E_{n} / T}+1}
$$

Un méthode itératif est utilisé pour résoudre $H_{M F}$. Cette convergence est atteindue quand la différence de tout paramètres d'ordre est moins de $10^{-3}$ entre les itérations consécutives et par fois $10^{-4}$ si nécessaire. Aprés les solutions sont obtenues, nous pouvons calculer les paramètres d'ordre avec cettes formules suivantes:

$$
\begin{aligned}
\Delta_{i} & =\sum_{\mu}\left(g_{i, \mu}^{t} g_{i+\hat{x}, \bar{\mu}}^{t} \Delta_{i, i+\hat{x}, \mu}^{v}+g_{i, \mu}^{t} g_{i-\hat{x}, \bar{\mu}}^{t} \Delta_{i, i-\hat{x}, \mu}^{v}-g_{i, \mu}^{t} g_{i+\hat{y}, \bar{\mu}}^{t} \Delta_{i, i+\hat{y}, \mu}^{v}-g_{i, \mu}^{t} g_{i-\hat{y}, \bar{\mu}}^{t} \Delta_{i, i-\hat{y}, \mu}^{v}\right) / 8, \\
m_{i} & =\left(\sqrt{g_{i, i+\hat{x}}^{s, z}}+\sqrt{g_{i, i-\hat{x}}^{s, z}}+\sqrt{g_{i, i+\hat{y}}^{s, z}}+\sqrt{g_{i, i-\hat{y}}^{s, z}}\right) m_{i}^{v} / 4, \\
K_{i, i+\hat{x}}= & \frac{1}{2} \sum_{\mu} g_{i, i+\hat{x}, \mu}^{t}\left\langle c_{i \mu}^{\dagger} c_{i+\hat{x} \mu}\right\rangle+g_{i+\hat{x}, i, \mu}^{t}\left\langle c_{i+\hat{x} \mu}^{\dagger} c_{i \mu}\right\rangle, \\
K_{i, i+\hat{y}}= & \frac{1}{2} \sum_{\mu} g_{i, i+\hat{y}, \mu}^{t}\left\langle c_{i \mu}^{\dagger} c_{i+\hat{y} \mu}\right\rangle+g_{i+\hat{y}, i, \mu}^{t}\left\langle c_{i+\hat{y} \mu}^{\dagger} c_{i \mu}\right\rangle, \\
K_{i} & =\left(K_{i, i+\hat{x}}+K_{i, i-\hat{x}}+K_{i, i+\hat{y}}+K_{i, i-\hat{y}}\right) / 4
\end{aligned}
$$

où $\Delta_{i}$ et $m_{i}$ sont les paramètres d'ordre de couplage et spin à chaque site et $K$ est l'ordre de leap pour la symmétrie. Le UPOP aussi joue un role important dans notre article. Pour des modèles uni-directionelles, nous calculons $\overline{\Delta_{x}}$ and $\overline{\Delta_{y}}$ :

$$
\begin{aligned}
& \overline{\Delta_{x}}=\sum_{\mathbf{K}} \sum_{i}^{N_{x}} \Delta_{i i+\hat{x}}^{\mathbf{K}} / N_{x} / M_{c} \\
& \overline{\Delta_{y}}=\sum_{\mathbf{K}} \sum_{i}^{N_{x}} \Delta_{i i+\hat{y}}^{\mathbf{K}} / N_{x} / M_{c}
\end{aligned}
$$

où $N_{x}$ est la taille de treillis dans la direction d'x et $M_{c}$ est la taille de supercell. K est le vecteur d'onde pour différent supercell et sa forme sera demonstré aprés. $\hat{x}(\hat{y})$ est le vecteur d'unité à la direction d'x(y). Aprés nous obtenons la valeur moyenne de couplage, 
nous pouvons afficher UPOP:

$$
U P O P=\frac{\left|\overline{\Delta_{x}}\right|+\left|\overline{\Delta_{y}}\right|}{2}
$$

\subsubsection{Fonctions de Green et LDOS}

Car les fonctions Green des modèles dont nous sommes intéresés sont tout à une direction, nous pouvons appliquer l'invariance translationelle à la direction d'y lors que nous mettons nos modèles suivi la direction d'x pour diminuer le temps de calcul. Nous transformons nos création/anéantissement opérateur à la base de $\left(i_{x}, k\right)$ :

$$
c_{i, \mu}^{\dagger}=\frac{1}{\sqrt{N}} \sum_{k} c_{i_{x}, \mu}^{\dagger}(k) e^{-i k R_{i_{y}}}
$$

Nous pouvons changer notre Hamiltonian dans un treillis en 1D. Avec cette transformation, nous pouvons afficher pour des treillis deux fois plus grand. Pour les symboles, $N$ répresente la taille en direction d'y, $R_{i_{y}}$ est la composant d'y du vecteur original au $i$. $c_{i_{x}, \mu}^{\dagger}(k)$ est l'opérateur de création en système de quasi-1D pour l'élan $k$. Alors l'Hamiltonian devient:

$$
\begin{aligned}
H= & \sum_{\left\langle i_{x}, j_{x}\right\rangle, k, \mu} H_{i_{x} j_{x} \mu}(k) c_{i_{x} \mu}^{\dagger}(k) c_{j_{x} \mu}(k)+h . c . \\
& +\sum_{\left\langle i_{x}, j_{x}\right\rangle, k, \mu} \sigma D_{i_{x} j_{x} \mu}^{*}(k) c_{i_{x} \mu}(k) c_{j_{x} \bar{\mu}}(-k)+h . c . \\
& -\sum_{i_{x}, k, \mu} \epsilon_{i_{x} \mu} n_{i_{x} \mu}(k)
\end{aligned}
$$

où

$$
H_{i_{x} j_{x} \mu}(k)=\sum_{i_{y}} H_{i_{x} i_{y} j_{x} 0 \mu} e^{-i k R_{i_{y}}}
$$


l'expression similaire existe pour $D_{i_{x} j_{x} \mu}(k)$ et $\epsilon_{i_{x} \mu}$. Avec des fonctions propres, la fonction Green peut être exprimé par:

$$
\begin{aligned}
& G_{i j \mu}(\omega)=\frac{1}{N} \sum_{k} g_{i j \mu}^{t} G_{i_{x} i_{y} \mu}(k, \omega) e^{i k\left(R_{i_{y}}-R_{j_{y}}\right)} \\
& G_{i_{x} i_{y} \mu}(k, \omega)=\sum_{n>0}\left[\frac{u_{i_{x} \mu}^{n}(k) u_{j_{x} \mu}^{n *}(k)}{\omega-E_{n \mu}(k)+i 0^{+}}+\frac{v_{i_{x} \mu}^{n *}(k) v_{j_{x} \mu}^{n}(k)}{\omega+E_{n \bar{\mu}}(k)+i 0^{+}}\right]
\end{aligned}
$$

Il y a plusier formes de $0^{+}$mais pricipalement il est égal de $0.01 t$ si ne pas mentionné particulièrement. Pour calculer l'LDOS à la pointe mesuré par STM, nous changons la base et obtenons la fonction Green continu par [119]:

$$
G_{\mu}(\mathbf{r}, \omega)=\sum_{i j} G_{i j \mu}(\omega) W_{i}(\mathbf{r}) W_{j}^{*}(\mathbf{r})
$$

où $W_{i}(\mathbf{r})$ est la fonction Wannier à la location $i$ et $\mathbf{r}$ est un vecteur continu dans l'éspace réel en 3D. La fonction Wannier utilisé d'ici est créé par Wannier 90 [120] et elle est similaire de la forme utilisé par [121]. Il faut faire attention que la fonction Green locale contient d'influence à tout la site dans le treillis . L'LDOS est calculé facilement par:

$$
\rho_{\mu}(\mathbf{r}, \omega)=-\frac{1}{\pi} \operatorname{Im}\left[G_{\mu}(\mathbf{r}, \omega)\right]
$$

Dans beaucoup de travaille avant [51, 53, 32], le facteur de forme a été obtenu par la transformation Fourier de leap le plus à côté $\chi_{i, i+\hat{x}(\hat{y})}$ qui peut être pensé comme une mésure de densité de charge à la location d'oxygène de la site $i$. Nous pouvons exprimer le facteur de forme $s-, s^{\prime}-$, et $d$ comme la suivant.

$$
\begin{aligned}
& D(\mathbf{q})=F T\left(\widetilde{\chi}_{i, i+\hat{x}}-\widetilde{\chi}_{i, i+\hat{y}}\right) / 2 \\
& S^{\prime}(\mathbf{q})=F T\left(\widetilde{\chi}_{i, i+\hat{x}}+\widetilde{\chi}_{i, i+\hat{y}}\right) / 2 \\
& S(\mathbf{q})=F T\left(1-\widetilde{\delta}_{i}\right)
\end{aligned}
$$

où FT répresnte la transformation Fourier et $\sim$ signifie que la moyenne spatiale de quantité correspondant était soustraite au but de soulinger la modulation. Clairement, ce quantité 
n'a pas dependance d'energie mais l'expérience de STM utilise des techniques [28] à traiter le facteur de forme et a trouvé une dependance en biais [1]. Avec cette info. de STM, nous pouvons suivre une manière similaire. Nous obtenons LDOS $Z$-map d'abord sur une plane locale en haut de $\approx 5 \AA$ de plane de $\mathrm{BiO}$.

$$
\rho^{Z}(\mathbf{r}, \omega>0)=\frac{\sum_{\mu} \rho_{\mu}(\mathbf{r}, \omega)}{\sum_{\mu} \rho_{\mu}(\mathbf{r},-\omega)}
$$

Ensuite, nous prennons de région séparément au tour d'atome avec la taille de région similaire de ce que utilisé par l'expérience [1], et attribuons au sub-treillis $Z$-maps $C u^{Z}(\mathbf{r}, \omega), O_{x}^{Z}(\mathbf{r}, \omega)$ et $O_{y}^{Z}(\mathbf{r}, \omega)$. Nous avons notifié que le facteur de forme n'est pas sensitif de la taille de treillis. Ici, $\mathrm{x}$ et y répresentent deux atomes d'oxygène en direction horizontale ou verticale. Si l'on prend une conbination correctement nous pouvons obtenir des facteurs de forme $s-, s^{\prime}-$, et $d$ :

$$
\begin{aligned}
& D^{Z}(\mathbf{q}, \omega)=\left(\widetilde{O}_{x}^{Z}(\mathbf{q}, \omega)-\widetilde{O}_{y}^{Z}(\mathbf{q}, \omega)\right) / 2 \\
& S^{Z}(\mathbf{q}, \omega)=\left(\widetilde{O}_{x}^{Z}(\mathbf{q}, \omega)+\widetilde{O}_{y}^{Z}(\mathbf{q}, \omega)\right) / 2 \\
& S^{Z}(\mathbf{q}, \omega)=\widetilde{C u}_{x}^{Z}(\mathbf{q}, \omega)
\end{aligned}
$$

Une autre quantité important est la différence du phase de moyenne spatiale $(\Delta \phi)$ entre les chaînes d'energies positives et négatives pour le $d$-form facteur. Pour afficher $\Delta \phi$ en concordance avec le procédé d'expérience [80], nous filtrons le vecteur de $d$-form modulation $\left(Q_{d}\right)$ de la modélisaion de LDOS en energies positives et négatives par la filtre Gaussian. Alors nous prennons la transformation Fourier inversé pour obtenir $D(\mathbf{r}, \omega)$ et $\phi(\mathbf{r}, \omega):$

$$
\begin{aligned}
& D^{g}(\mathbf{q}, \omega)=\left(\widetilde{O}_{x}^{g}(\mathbf{q}, \omega)-\widetilde{O}_{y}^{g}(\mathbf{q}, \omega)\right) / 2 \\
& D(\mathbf{r}, \omega)=\frac{2}{(2 \pi)^{2}} \int d \mathbf{q} e^{i \mathbf{q r}} D^{g}(\mathbf{q}, \omega) e^{-\frac{\left(\mathbf{q}-\mathbf{Q}_{d}\right)^{2}}{2 \Lambda^{2}}} \\
& \phi(\mathbf{r}, \omega)=\tan ^{-1}(\operatorname{Im}[D(\mathbf{r}, \omega)] / \operatorname{Re}[D(\mathbf{r}, \omega)]) \\
& \Delta \phi=\langle\phi(\mathbf{r}, \omega)-\phi(\mathbf{r},-\omega)\rangle
\end{aligned}
$$


où $\widetilde{O} g(\mathbf{q}, \omega)$ et $\widetilde{O}_{y}^{g}(\mathbf{q}, \omega)$ sont le FT de modélisation de LDOS de sub-treillis pour l'oxygène d'x et y. Nous utilisons $\Lambda=1 / 2 N$.

\subsubsection{La spectre et le nombre de Chern}

Car nous allons chercher des charactéristiques en éspace de $k$, il est nécessaire d'appliquer le calcul de supercell [122]. Pour chaque cell nous avons $N_{x} \times N_{y}$ sites et alors nombre de cells en totale $M_{c}=M_{x} \times M_{y}$. Notre Hamiltonian est donc réduit de $2 M_{x} N_{x} \times 2 M_{y} N_{y}$ à $M_{x} \times M_{y}$ équations de matrix et nombre de site $2 N_{x} \times 2 N_{y}$ pour chaque cell. Le calcul est affiché pour chaque cell et la spectre est décrit avec nos fonctions propres $(u, v)$ comme:

$$
\begin{aligned}
A(k, \omega)= & \frac{1}{N} \sum_{i j, n+} f\left(-E_{n}\right)\left(e^{i \mathbf{k} \cdot\left(\mathbf{r}_{i}-\mathbf{r}_{j}\right)} g_{i j \uparrow}^{t} u_{i, n}^{\mathbf{K} *} u_{j, n}^{\mathbf{K}} \delta\left(\omega-E_{n}\right)\right. \\
& \left.+e^{i \mathbf{k} \cdot\left(\mathbf{r}_{j}-\mathbf{r}_{i}\right)} g_{i j \downarrow}^{t} v_{i, n}^{\mathbf{K}} v_{j, n}^{\mathbf{K} *} \delta\left(\omega+E_{n}\right)\right) \\
& +\frac{1}{N} \sum_{i j, n-} f\left(E_{n}\right)\left(e^{i \mathbf{k} \cdot\left(\mathbf{r}_{i}-\mathbf{r}_{j}\right)} g_{i j \uparrow}^{t} u_{i, n}^{\mathbf{K} *} u_{j, n}^{\mathbf{K}} \delta\left(\omega-E_{n}\right)\right. \\
& \left.+e^{i \mathbf{k} \cdot\left(\mathbf{r}_{j}-\mathbf{r}_{i}\right)} g_{i j \downarrow}^{t} v_{i, n}^{\mathbf{K}} v_{j, n}^{\mathbf{K} *} \delta\left(\omega+E_{n}\right)\right)
\end{aligned}
$$

où $\mathbf{k}=\mathbf{k}_{0}+\mathbf{K}$ dans le même temps $\mathbf{k}_{0}=2 \pi\left(\frac{n_{x}}{N_{x}}, \frac{n_{y}}{N_{y}}\right)$ où $n_{x} \in\left[-N_{x} / 2+1, N_{x} / 2\right], n_{y} \in$ $\left[-N_{y} / 2+1, N_{y} / 2\right]$, et $\mathbf{K}=2 \pi\left(\frac{n_{x}^{c}}{M_{x} N_{x}}, \frac{n_{y}^{c}}{M_{y} N_{y}}\right)$ où $n_{x}^{c} \in\left[0, M_{x}-1\right], n_{y}^{c} \in\left[0, N_{y}-1\right]$. $f\left(E_{n}\right)$ est la distribution de Fermi-Dirac et $n+(n-)$ signifie la sommation d'energies positives(négatives). $\delta\left(\omega-E_{n}\right)$ est la Lorenzian et a la forme suivante:

$$
\delta\left(\omega-E_{n}\right)=\frac{1}{\pi} \frac{\Gamma}{\Gamma^{2}+\left(\omega-E_{n}\right)^{2}}
$$

Ensuite, pour chaque band aplati par le champ magnétique ses numbres de Chern sont définis par l'intégration de courbure Berry partout la zone Brillouin [123]:

$$
\begin{aligned}
& C_{n}=\frac{1}{2 \pi} \sum_{\mathbf{k} \in B Z} \nabla_{\mathbf{k}} \times \vec{A}_{n}(\mathbf{k})=\frac{1}{2 \pi} \sum_{\mathbf{k} \in B Z} \vec{B}_{n}(\mathbf{k}) \\
& =\frac{-i}{2 \pi} \sum_{m \neq n} \sum_{\mathbf{k} \in B Z} \frac{\left\langle n \mathbf{k}\left|J_{x}\right| m \mathbf{k}\right\rangle\left\langle m \mathbf{k}\left|J_{y}\right| n \mathbf{k}\right\rangle-\left(J_{x} \leftrightarrow J_{y}\right)}{\left[E_{n}(\mathbf{k})-E_{m}(\mathbf{k})\right]^{2}}
\end{aligned}
$$


où $\vec{A}_{n}(\mathbf{k})=-i\left\langle n \mathbf{k}\left|\nabla_{\mathbf{k}}\right| n \mathbf{k}\right\rangle$ est le champ de vecteur de Berry pour nth band, et $\vec{B}_{n}(\mathbf{k})$ est le champ magnétique relatif. La courante $\mathbf{J}=\left(J_{x}, J_{y}\right)$ est obtenue par $J=\nabla_{\mathbf{k}} H$.

\subsection{Supraconduteur à haute température}

Le premier système où nous avons appliquer notre méthode d'RMFT est ce que pour la mystique de supraconducteur à haute température. Après sa découverte il y a 30 ans, on ne peut pas encore définir une théorie pour expliquer sa micro-mécanique de manière appropriée. Cependant, avec des équipements avancés, on peut faire des expériences correctement et obtenir des résultats exacts. Ces preuves nous facilitent l'élaboration d'une bonne théorie, même s'il est aussi très difficile d'inclure tous les phénomènes ensemble. Nous avons obtenu des résultats et par rapport aux expériences et nous allons faire d'un petite sommaire ici.

\subsubsection{Charactéristique en éspace réel}

Dans l'approximation de Gutzwiller qui est non homogène, le $d$-wave homogène supraconducteur a une energies plus bas que celles-là d'autre modèles avec des instabilités partout le niveau de doping. Alors PDW n'est pas l'état normal de $t-t^{\prime}-J$ Hamiltonian. Cependant, la différence d'energies entre l'état homogène et l'autre états est petite [40]. Par conséquant, quand quelque influences minoritaires comme l'interaction d'électronphonon sont inclues, il est possible que ces modèles avec des modulations peuvent obtenir des énergies plus bas [43, 126]. En fait, la nature de courte portée de ces états, observé par les expériences de STM [69] et REXS [68] suggére que des désordres peuvent jouer un rôle important. Différent désordres locals peuvent aussi stabiliser des états avec la modulation. Cette réalité laisse que des modèles différentes peuvent être observé par les expériences. Le clé est les facteurs Gutzwiller. Ils changent forcément avec la variation de doping et fournissent un énvironnement pour la dégénérescence de modèle différente, montré par 3.2(a) pour tout le doping. La différence entre des énergies maintient constantes partout le niveau de doping. Par conséquant, l'addition d'un champ magnétique 
entre $10 \mathrm{~T}$ ou $1 \mathrm{meV}$ à chaque site peut stabiliser un ordre de charge en longue portée. Un résultat d'observation d'une ordre de charge de YBCO avec une corrélation longuee, dnas un champ magnétique de $30 T$ sert comme une preuve de ce phénomène [127, 128].

Nous avons trouvé que au doping certain, l'état de nPDW avec des vecteurs d'onde Q autour $(0.3,0)$ existe. Si nous maintenons la même taille de treillis $(N \times N)$, l'ordre de charge change un peu avec un $Q=(Q, 0)$ différent, car $Q$ est rélative de $1 / N$. Cependant, LDOS, facteur de forme, et différence de phase ne sont pas sensitifs de tellement petite change. Tout ces états avec $Q$ similaire ont des énergies trés proche et donc, au niveau d'approximation de Gutzwiller, nous ne pouvons pas distinguer la rélation entre $Q$ et doping. Mais la dépendance du facteur de forme et de la différence de phase est similaire partout les vecteurs $Q$, structures de bande, $t^{\prime}$, et dopings. Nous avons toujour obtenu la même $d$-forme facteur aux energies hautes et un changement de phase de $\pi$ en différence de phase moyenne au-délà d'une énergie certaine.

Cette analyse presenté en partie 3 que PDW et CDW sont surprimés artificiellement suggére que la rôle de PDW est nécessaire au but d'expliquer des charactéristiques méntionnées, particuliellement pour la dépendance du factuer de forme et de la différence de phase, montré par l'expérience sur BSCCO.

Il est important à noter que la dépendance du facteur de forme dans notre théorie est un résultat claire de la corrélation électronique sur le plan de $\mathrm{CuO}$. Elle était observée dans la spectre de X-ray que le plan de YBCO, par exemple, fixe une modèle du déplacement de $\mathrm{O}$ qui imite le facteur de $d$-forme [129], et suggére que cette modèle s'impose pour une conductance locale. Cependant, il est difficile à découvrir comment cet effet est sensitive en biase, observé par l'expérience. Autrefois, comment l'autre facteur de forme peut influencier quelque scénarios maintient une mystère.

\subsubsection{Charactéristique en éspace d'élan}

Si nos facteurs Gutzwiller qui incluent l'effet de la physique de corrélation forte ne sont pas beaucoup influenciés par la température faible comparé à celle ambiante, nous pouvons utiliser les même facteurs pour notre RMFT aux températures non-zéro de modèle $t-t^{\prime}-J$. 
Aux températures faibles sous la région de la phase de SC avec un UPOP non-zéro, une solution spéciale qui s'appelle nPDW a été proposé par nous. Elle a deux gaps de d paire, comme démontré par l'expérience de ARPES. Quand la dopage est plus bas, le gap à l'antinode dévient plus grand mais celui à la node ne change pas beaucoup. Il y a une asymétrie montré par ARPES, l'asymétrie de particule-trou à la région de antinode a été aussi produit par nous. L'état de nPDW a des charactéristiques spécieux même s'il est uni-dimensionel. Il a UPOP qui est symétrique en d paire. C'est uncroyable que même si les valeurs de paire semblent aléatoire à chaque bond(Fig. 3.3(a)), sa moyenne nous montre une symétrie de d-paire. La spectre aux $(\pi, 0)$ et $(0, \pi)$ sont tellement différentes mais ses valeurs s'approchent [4]. Avec nos découvertes avant pour l'expérience de STS, nous avons obtenu une scénario constante comparé aux experiéences.

Ici il est important à mentionner que l'état de nPDW que nous avons choisi est entre beaucoup de solutions possibles avec périodes différantes. Mais ses charactéristiques sont similaire seuf la différence de période. Avec la comparaison d'energie c'est claire que $d$ wave SC est l'état fondamental de la modèle $t-t^{\prime}-J$ résoudu par RMFT. Cépendant, comme mentionné avant, n'importe quand nous incluons des interactions faibles, par exemple l'interaction d'électron-phonon, la force de Coulomb, ou des impuretés [42, 47], nPDW peut être stabilisé et dévient l'état fondamental. Même si nous considérons simplement la modèle $t-t^{\prime}-J$, nos états sont aux minima locales. Donc nous pouvons analyser ses excitements d'énergies faibles.

Une l'autre chose est que les expériences comme notre théorie ont trouvé beaucoup de genre de CDW différent. Pour la famille de $L a_{2-x} B a_{x} C u O_{4}$, la période de CDW diminue avec la dopage mais elle augmente pour YBCO et BSCCO [19]. Ces deux sont appellés CDW1 et CDW2 séparément, à l'une article [36]. Il y a aussi CDW3 ou le CDW évoqué par le champ magnétique. Notre travail est pour la plupart sur CDW2 et CDW1 est peut-être pour l'état de rayure [47].

Quand la température augmente, le UPOP de nPDW commence à diminuer et il dévient faible aprés $T_{p 1}$. Cette conduite soutient notre théorie que ces états sont simplement minima locales. Aprés cette température, les états déviennent IPDW qui ont encore des modu- 
lations disproportionnées de CDW, BDW, et PDW mais sans UPOP. Les charactéristiques entre les deux états sont similaire seuf que la FFT de CDW n'a pas un sommet où PDW est au maximum [88]. C'état ne change pas rapidement jusqu'à la température de $T_{p 2}$ et tous solutions avec modulation disparaissent. $T_{p 2}$ diminue quand la dopage augmente. Fig. 3.16(a) montre que $T_{p 2}$ est proportionnelle aux températures de PG $T^{*}$. C'est déjà intéressant que notre théorie de champ moyen peut attirer ce résultat.

En plus, nos analyse montre que l'état de IPDW à côté de la région de node a un arc de Fermi avec une fraction de DOS de la surface de Fermi sans paire. Il y a encore un gap forcé a la région d'anti-node montré à Fig. 3.16(b). Le DOS ou longueur d'arc Fermi augmente avec la dopage comme les découvertes par ARPES [21] et NMR [3]

À notre calcul nous avons obtenu l'état de d paire SC aux température plus de $T_{p 2}$. Cependant, comme noté avant, ce résultat est un conséquence que l'état de d paire est en fait l'état fondamental surtout l'espace Hilbert. Nous pensons que si nous incluons IPDW en directions x- et y-, l'entropie haute peut stabiliser l'énergie libre et laisser cet état fondamental. Donc aprés $T_{p 2}$ nos solutions déviennent encore d paire SC car l'exactitude de RMFT sont limité et ils peut-être n'ont pas l'importance physicale.

Il faut soulinger que IPDW est aussi un SC FFLO [85, 86] avec un élan non-zéro si la cohérence de phase existe. Mais actuellement il y a des domaines sur les matérieux avec CDW, BDW, et PDW différents [132]. VMC [43] a montré que l'énergie d'état avec des domaines à la même temps peut obtenir une energies plus bas et dévient l'état fondamental. La phase de PG est conprise souvant avec des tourbillons [133, 134]. L'inclusion de phase différente pour chaque domaine peut aider montrer la scénario de supraconductivité plus clairement [88, 93, 94]. Cependent, le PDW décrit d'ici doit être une base de solution différente découverte par l'expérience.

Une l'autre chose importante est que avec le champ magnétique, un CDW peut être créé au milieu de tourbillon pour YBCO [135, 136, 137]. Quelque CDWs sont 3D [127]. Pour la famille de BSCCO différent résultat était découvert. Seuf CDW, la modulation d'échiquier soie aussi possible [138]. Pour $B i_{2} S r_{2-x} L a_{x} C u O_{6}$, NMR [139] a montré que quand un champ magnétique est mis de 10T, il est suffisant pour un CDW sans spin 
à la phase de PG. Car tant petit champ n'influence pas nos états, nous pensons qu'ils sont ceux de $\mathrm{Bi}_{2} \mathrm{Sr}_{2-x} \mathrm{La}_{x} \mathrm{CuO}$. Le conformément montré à Fig. 3.16(c) soutient notre théorie.

\section{3 Électrons corrélé dans un champ magnétique}

Le deuxième système qui nous intéresse est le mouvement d'électron dans un champ magnétique fort. Le papillon d'Hofstadter et son modèle, l'Hamiltonian de Harper-Hofstadter ont obtenu un grand succès à décrire la mécanique d'électrons libres aux treillis. Donc il est ainsi intéressant de se demander ce qu'il se passera si nous remplaçons des électrons libres avec ceux qui s'interagissent. D'ailleurs, $t-J$ Hamiltonian s'utilise comme bon modèle à le découvrir. Nous allons comparer nos résultats avec ceux de la diagonalisation exacte. Nous proposerons des découvertes intéressantes qui désormais seront réalisées par l'expérience d'atome froide.

Ensemble avec l'ordre de charge et bonde, il est particulièrement intéressant de trouver l'émergence d'état de FQH avec l'ordre de topologie. À demi-remplissage ( $\rho=1 / 2)$ la liquide de chiral spin topologique était construite comme la projection Gutzwiller de fonction d'onde non-interagissant avec une bande pleine de nombre Chern \pm 1 [141, 142 , 143]. Une construction d'état topologique est aussi appliqué loin de la dopage de demiremplissage à la dopage et/ou au densité d'électron plus bas. Et il peut être montré par la projection Gutzwiller. À cette cas, notre manière peut atteindre des situations qui ont les énergies plus bas avec des ordres topologiques.

Notre première conclusion est que pour les états $\nu=1$ et $\nu=7$ dans la region de l'effet de quantum Hall avec du nombre entier il y a un écart et donc il n'est pas possible que des instabilité d'ordre de topologie existent. Nous avons encore des états qui ont ses électrons avec la même spin de $\Phi=7 / 16$. La manière le plus simple pour distinguer ces qui sont les états de $\mathrm{FQH}$ dans une symmetrie avec des conditions de la frontière périodique est la dégénérescence d'état fondamental [144]. Nous nous attendons deux états qui ont les énergies à côté et séparés par un écart de les autre états(si un système réalise un état Langhlin de $\nu=1 / 2$, sa dégénérescence topologique est deux, par exemple). Figure A.2 
dans l'Appendix A montre la spectre d'énergie pour chaque état que nous avons, sous $S_{z}$ qui est un bon nombre du quantum d'ici. Nous avons découvert que GSD n'apparaît pas même si pour quelque solutions ses énergies d'état fondamental sont proche de ce lui de la deuxième état. Par exemple, pour $\nu=2 / 7$ et $S_{z}=0$ ils ont deux états avec ses énergies tellement similaire(et pétit aussi), mais un de deux est $S=0$ et l'autre $S=2$. Par conséquent, ces états ne sont de la dégénérescence. En plus, nous avons trouvé que ces deux états ont des nombres Chern qui sont pair et donc ils no sont pas des états de FQH.

La raison pourquoi il est difficile pour que ces états ne peuvent pas réaliser un état de FQH est composé par deux parties: L'interaction Hubbard est la force primaire et elle est extrêmement locale. Pour l'effet FQH, le terme d'interaction, qui est projecté comme un état des particules séparés sur un niveau Landau, est décrite comme une pseudopotential Haldane. Une interaction extrêmement locale est égale de $V_{0}$ pseudopotential, qui peut créer un état bosonique de FQH. Mais pour l'état fermionique le plus simple, il faut au moins inclure une pseudopotential $V_{1}$. Cependant, comme ce qui étaient découverts du isolateur Chern [145], cette interaction extrêmement locale transforme à une potential $V_{0}$ sur une bande avec non-zéro nombre de Chern.

\subsection{Conclusion}

Les résultats montrés avant sont tous en base d'une théorie du champs moyen rénormalisé [31] et GWA [56] pour la modèle de $t-J$ ou $t-t^{\prime}-J$. Même s'ils ne fournissent pas des valeurs exactes, opposite de beaucoup d'autre manières qui peuvent, nos résultats montrent qu'ils réflechissent la physique de la corrélation forcée. Pour exprimer la physique de cuprate, tout d'abord, cette corrélation fournit une rénormalisation Gutzwiller qui se trouve à chaque emplacement qui produit beaucoup de la solution exotique de PDW stripe et/ou CBs combinées avec des modulations de densité de charge et/ou de spin. Ces résultats montrent que nos solutions agréent avec des expériences clé [15, 24, 25]. Car nos rénormalisations sont extrêmement locales, l'effet de surface Fermi ou vecteur d'onde $k_{F}$ est absent. Notre modèle n'est pas besoin du mouvement sautillant de la deuxième ou 
troixième côté pour créer une surface Fermi avec vecteur de modulation ou "hot spots" [33, 51, 91]. Par conséquent, dans notre théorie, il n'y a une unique vecteur d'onde pour le densité du charge ou spin et CB. Même si nous avons obtenu beaucoup de résultat pour $4 a_{0}$, notre recherche aussi montrait la possibilité de l'autre période d'onde comme $5 a_{0}$ ou $3 a_{0}$. États avec période longue doit être possible et ses énergies doivent avoir des valeurs similairees [46, 54]. Si nous permettons la combination de plusieur période, nPDW peut être découvert et nous avons des états avec période incommensuratee.

Une conséquence importante que pour avoir des états qui ont la période de modulation différente d'une Hamiltonian commum, les états doivent n'être pas les "competing states" que nous avons compris. Ils ne restent pas une minimum d'énergie locale et ils sont sensitifs. Il s'agit que les états peuvent se transformer facilement. Un bon exemple est que comme nous avons montré, nPDW est en fait la combination de AP-CDW et d-SC. Pour l'autres exemples de stripe mixés, mis à la Table 3.1, ils peuvent aussi être créés. Pour la matériau de cuprate, il y a beaucoup d'autre interactions par ailleur de nos $t$ et $J$ qui peuvent changer la préférance d'état. Par exemple, une interaction faible d'électron laisse la stripe de IP-CDW-SDW plus stabilisé par rapport aux autre états [42]. Si nous inclurons l'effet de surface Fermi, le résultat aussi changera. Cépendent, aucune de ces interaction est plus important que ce qui vient de la physique de Mott avec la rénormalisation pour produire la modulation d'ordre.

Avec le saut seconde $t^{\prime}$, nous avons montré que il y a des solutions qui ont les énergies basses avec des ordres du charge ou pairing qui est proportionné ou disproportionné de la Hamiltonian $t-t^{\prime}-J$. Nos solutions ne sont pas les états fondamentals mais ses énergies sont trés proche de celle-là d'état supraconductivité homogène. En plus, un état qui s'appelle nPDW est entrelacé avec des modulations du charge ou pairing et nous montre des charactéristiques qui sont similaires par rapport aux expériences de STM sur la surface de BSCCO et NaCCOC. Il y a en fait des phénomènes de cuprate observé qui intéressent les scientifiques plus de 30 ans mais nous ne pouvions pas agréer entre nos explications. Une phénomène specielle est que le même spectre et conductance sont observé par STM des matérieux BSCCO ou NaCCOC qui sont classé comme les cuprates différents. En 
calculant cette phénomène, nous avons inclut la méthode par Choubey et al. [119] en utilisant la fonction de Wannier qui peut afficher les fonctions d'onde aux tous positions en $3 \mathrm{D}$, en incluant les positions de quelque angstrom en haute de celle où la pointe de STM est placé. Cette technique nous a fourni une manière de comparer nos résultats avec ceux de la expérience.

En plus, la dépendence de intra-unite cell $d-, s^{\prime}-$ et $s$ facteur du form et ses différences du phase étaient obtenus dans l'état de nPDW et ont permis une trés boone correspondance avec l'expérience de STM. L'énergie du facteur de form avec la symmetrie $d$-onde change avec la dopage juste comme le résultat observé dans pseudogap. Notez que sauf nous, théories avant d'ordre du charge de la modèle $t-t^{\prime}-J$ discutaient seulement l'ordre du charge commensurate et exprimaient les résultats observés avec des restrictions.

Un de nos surprises que nous avons trouvé est que nous n'avions pas des paramètres accordable dans nos calculations. Nous pouvions obtenir des valeurs côté de celles de l'expérience et aussi nos calculs sont similaire par rapport aux autre travails qui sont plus compliqué que notre façon qui est simplement du champs moyens. Un raison majeur est que le GWA est tellement effectif en réalisant la physique de la modèle $t-J$. Basé à ce raison, nous pouvions fournir une figure trés simple sur la diagramme du phase de cuprate. En commercant de la fourrage moitié, cette modèle est RVB proposé par Anderson [12] qui est plus important en isolant de Mott. RVB a le pairing de $d$-onde et d'ordre du bonde qui sont entrelacé. Mais sans l'ordre du charge ils sont en fait des paramètres variationnelles que nous avons définies en Eq. 6.4. Quand nous ajoutons des trous d'électron aux matérieux, RVB prévient le mouvement électronique et donc prévient la modulation. Mais aprés cette localisation est détruite à cause de la disparition de antiferromagnetique, la système devient un état avec PDW et la modulation du charge entrelacé avec RVB(des ordres du pairing et bonde). Ces états ont un écart dans la région d'anti-node et une arc de Fermi à côté de node. Dans une arc de Fermi, seulement partie de DOS maintient. Quand il y a plus de trou d'électron et ces modulations sont détruits, nous perdrions la physique de Mott et une FLS se re-installerait [150]. Alors, ces états deviennent un SC régulière dans la température basse même s'il est petit par rapport à l'ampleur de la modulation du 
pairing. Bien sûr, la fluctuation du phase joue une rôle important quand la température augmente [36, 93] et notre théorie du champs moyens a besoin d'être amélioré.

La théorie que nous avons proposée est dépendant de la présence de nPDW dans le phase de PG. Il y a un façon d'examiner notre théorie d'ailleur l'expérience de STS [1], qui concerne la variation du phase rapide dans un petit région et aussi ses mesures sont surtout dans une température haute. Pour un état de nPDW dans la direction $x$, l'ampleur d'écart dans la direction $y,(0, \pi)$, est approximativement la même [4] par rapport à celui de la $x$ direction, $(\pi, 0)$, comme en montrant dans Fig. 3.11 et 3.12. Il est au contraire de ce que nous avons espéré si nous aurions simplement une modulation dans la direction $x$, où l'écart créé par la pliage du zone doit être plus grand. L'asymmetrie de la surface de Fermi aux $x$ et $y$ directions, montré dans Fig. 3.15(f), sert comme une manière de distinguer l'arc de la surface de Fermi [80, 81]. Nous pouvons aussi examiner l'asymmetrie de ParticleHole dans PG. IPDW a des charactéristiques similaire de ceux de nPDW sous mesure par ARPES [82]. L'asymmetrie de Particle-Hole est observé loin d'arc de Fermi et il est une indication de Cooper pairs avec une élan non-zéro [39].

Particulièrement, nous avons discuté la possibilité que des impurité stabilisent l'ordre du charge, en créant une modulation en 1D sur BSCCO et NaCCOC, observé par STM. C'état fondamental agrée avec des résultats proposeé par l'expérience de resonant X-ray scattering [68] qui portent des charactéristiques d'ordre du change dans une période courte. Dans tant système, un champs magnétique peut surprimer la supraconductivité et finalement faciliter la création des ordres CDW ou PDW qui ont des périodes longues. Il était montré que avec un champs magnétique de $10 T$, il est suffisant de créer des ordres longues de CDW ou PDW [127, 139]. Car $10 T$ est petit, il est possible de créer des ordres longues en laissant un cuprate sur une couche stressé à une direction. Nous avons examiné ce cas avec le saut en direction $x$ plus petit que ce qui est en direction $y$. Le résultat montrait que l'énergie de nPDW pour $\delta=0.08$ est maintenant plus petite que ce d'état uniform si $t_{x}$ $<0.84 t_{y}$. Il a agréé aux résultats de Yang [40] pour $\delta=0.08$ au état de stripe. Dans un matériel réel, nous avons besion de petit stress en stabilisant un IPDW/nPDW. Car nPDW dans la direction $x$ sont plus stable, la système favorise la création de nPDW en $x$-direction 
plus que ce de nPDW en $y$-direction et donc une modulation uniforme même si $T>T_{c}$ pour IPDW. Il est aussi possible que le IPDW avec la cohérence du phase peut devenir un état nouveau dans une température spécifique. Même si nous perdons $t_{x}<t_{y}$, comme démontré par Fig. 3.11 et 3.12, les spectre proche de $(\pi, 0)$ et $(0, \pi)$ sont trés différents. Avec $t_{x}<t_{y}$, le UPOP a la symmetrie $s^{\prime}+d$ avec pairing plus grand dans $x$-direction que $y$-direction. D'ailleur, l'écart d'énergie proche de $x$ anti-node devient plus petit mais ce de $y$ anti-node devient plus grand. Cette phénomène peut être découvert par ARPES dans la système.

En plus, motivé par le développement d'expérience et technique numérique, nous examinions la modèle de Harper-Hofstadter sous la présence d'une corrélation forte, qui est égale de la modèle $t-J$ sous un champs magnétique. Avec notre manière de RMFT et calcul de Lanczos ED, nous avons essayé trouver des états nouveaux des système fermionique. Particulièrement, nous faisions attention du phase de CFPs et fermionique. Même si nous n'avons pas réussi observer un phase topologique, des états qui n'est pas banals avec non-zéro nombre de Chern sont découverts sous la présence d'interaction. Nous trouvions que CFPs qui est rélatif de la système de Hall quantique. CFPs dans une garniture $\nu$ qui n'est pas commensurate ont des instabilités. Pour nos systèmes qui ont ses électrons dans la même polarisation, qui s'arrivent dans une garniture basse, RMFT et ED s'agrée bien avec ses énergies et nombres de Chern calculés. En plus, nous montrions que l'effet d'une potential décalé peut détruire ses charactéristiques topologiques et nous l'exprimons en Appendix B. Notez que pour les phases proche de isolant de Mott, RMFT et ED ne s'agréent pas pour ses nombres de Chern ou énergies calculs. Il nous dit que l'interaction forte a mis des difficultés d'afficher ses charactéristiques topologiques. Par conséquent, il est intéressant de réaliser ces systèmes dans une expérience. Il était déjà possible à rechercher la modèle de Fermi-Hubbard avec le gas de Fermi qui est dégénéré avec ${ }^{6} \operatorname{Li}(37$, 38), dans un treillis optique [151]. Pour inclure le champs de gauge, nous pouvons utiliser deux lasers pour manipuler les saut en $x$ et $y$ directions et aussi ajouter un flux [97, 98]. Nous alors suggérons la combination de ces deux systèmes pour créer une scénario pour notre système décrite. Cependant, en comparé avec l'expérience d'Hamiltonian Harper- 
Hofstadter avec des interactions, les résultats s'agréent et il nous porte l'information que l'Hamiltonian $t-J$ est rélative quand nous voulons décrire la physique des fermions interactives sous un champs magnétique. Nous résultats sert comme une commerce de trouver la phénomène d'Hamiltonian Hofstadter corrélative forcément et motiver plus de recherche des théorie et expérience. 


\section{Bibliography}

[1] M. H. Hamidian, S. D. Edkins, C. K. Kim, J. C. Davis, A. P. Mackenzie, H. Eisaki, S. Uchida, M. J. Lawler, E. A. Kim, S. Sachdev, and K. Fujita. Atomic-scale electronic structure of the cuprate $d$-symmetry form factor density wave state. Nature Phys., 12:150-156, 2016.

[2] J. W. Alldredge, J. Lee, K. McElroy, M. Wang, K. Fujita, Y. Kohsaka, C. Taylor, H. Eisaki, S. Uchida, P. J. Hirschfeld, and J. C. Davis. Evolution of the electronic excitation spectrum with strongly diminishing hole density in superconducting $\mathrm{Bi}_{2} \mathrm{Sr}_{2} \mathrm{CaCu}_{2} \mathrm{O}_{8+\delta}$. Nature Phys., 4:319-326, 2008.

[3] S. Kawasaki, C. Lin, P. L. Kuhns, A. P. Reyes, and G. Zheng. Carrierconcentration dependence of the pseudogap ground state of superconducting $\mathrm{Bi}_{2} \mathrm{Sr}_{2-x} L \mathrm{La}_{x} \mathrm{CuO} \mathrm{O}_{6+\delta}$ revealed by ${ }^{63,65} \mathrm{Cu}$-nuclear magnetic resonance in very high magnetic fields. Phys. Rev. Lett., 105:137002, 2010.

[4] Gap values near the two antinodes my differ by $10 \%$ for different nPDW states. This is probably the accuracy of the mean-filed theory.

[5] J. G. Bednorz and K. A. Müller. Possible high $T_{c}$ superconductivity in the Ba-LaCu-O system. Z. Phys. B, 64:189-193, 1986.

[6] M. K. Wu, J. R. Ashburn, C. J. Torng, P. H. Hor, R. L. Meng, L. Gao, Z. J. Huang, Y. Q. Wang, and C. W. Chu. Superconductivity at $93 \mathrm{~K}$ in a new mixed-phase Y-Ba-Cu-O compound system at ambient pressure. Phys. Rev. Lett., 58:908, 1987. 
[7] H. Maeda, Y. Tanaka, M. Fukutomi, and T. Asano. A new high- $T_{c}$ oxide superconductor without a rare earth element. Jpn. J. Appl. Phys., 27:L209-L210, 1988.

[8] Z. Z. Sheng and A. M. Hermann. Bulk superconductivity at $120 \mathrm{~K}$ in the Tl-Ca/BaCu-O system. Nature, 332:138-139, 1988.

[9] A. Schilling, M. Cantoni, J. D. Guo, and H. R. Ott. Superconductivity above 130 $\mathrm{K}$ in the Hg-Ba-Ca-Cu-O system. Nature, 363:56-58, 1993.

[10] J. Bardeen, L. N. Cooper, and J. R. Schrieffer. Theory of superconductivity. Phys. Rev., 108:1175-1204, 1957.

[11] N. F. Mott. The basis of the electron theory of metals, with special reference to the transition metals. Proceedings of the Physicl Society. Series A, 62:416, 1949.

[12] P. W. Anderson. The resonating valence bond state in $\mathrm{La}_{2} \mathrm{CuO}$ and superconductivity. Science, 235:1196-1198, 1987.

[13] K. Yamada, C. H. Lee, K. Kurahashi, J. Wada, S. Wakimoto, S. Ueki, H. Kimura, Y. Endoh, S. Hosoya, G. Shirane, R. J. Birgeneau, M. Greven, M. A. Kastner, and Y. J. Kim. Doping dependence of the spatially modulated dynamical spin correlations and the superconducting-transition temperature in $\mathrm{La}_{2-x} \mathrm{Sr}_{x} \mathrm{CuO}$. Phys. Rev. $B, 57: 6165,1998$.

[14] P. Abbamonte, A. Rusydi, S. Smadici, G. D. Gu, G. A. Sawatzky, and D. L. Feng. Spatially modulated 'Mottness' in $\mathrm{La}_{2-x} \mathrm{Ba}_{x} \mathrm{CuO}$. Nature Phys., 1:155-158, 2005.

[15] Y. Kohsaka, C. Taylor, K. Fujita, A. Schmidt, C. Lupien, T. Hanaguri, M. Azuma, M. Takano, H. Eisaki, H. Takagi, S. Uchida, and J. C. Davis. An intrinsic bondcentered electronic glass with unidirectional domains in underdoped cuprates. Science, 315:1380-1385, 2007. 
[16] C. V. Parker, P. Aynajian, E. H. da Silva Neto, A. Pushp, S. Ono, J. Wen, Z. Xu, G. Gu, and A. Yazdani. Fluctuating stripes at the onset of the pseudogap in the high- $T_{c}$ superconductor $\mathrm{Bi}_{2} \mathrm{Sr}_{2} \mathrm{CaCu}_{2} \mathrm{O}_{8+x}$. Nature, 486:677-680, 2010.

[17] T. Wu, H. Mayaffre, S. Krämer, M. Horvatic, C. Berthier, W. N. Hardy, R. Liang, D. A. Bonn, and M. H. Julien. Magnetic-field-induced charge-stripe order in the high-temperature superconductor $\mathrm{YBa}_{2} \mathrm{Cu}_{3} \mathrm{O}_{y}$. Nature, 477:191-194, 2011.

[18] G. Ghiringhelli, M. Le Tacon, M. Minola, S. Blanco-Canosa, C. Mazzoli, N. B. Brookes, G. M. De Luca, A. Frano, D. G. Hawthorn, F. He, T. Loew, M. Moretti Sala, D. C. Peets, M. Salluzzo, E. Schierle, R. Sutarto, G. A. Sawatzky, E. Weschke, B. Keimer, and L. Braicovich. Long-range incommensurate charge fluctuations in $(Y, N d) \mathrm{Ba}_{2} \mathrm{Cu}_{3} \mathrm{O}_{(6+x)}$. Science, 337:821-825, 2012.

[19] R. Comin, A. Frano, M. M. Yee, Y. Yoshida, H. Eisaki, E. Schierle, E. Weschke, R. Sutarto, F. He, A. Soumyanarayanan, Y. He, M. Le Tacon, I. S. Elfimov, J. E. Hoffman, G. A. Sawatzky, B. Keimer, and A. Damascelli. Charge order driven by Fermi-arc instability in $\mathrm{Bi}_{2} \mathrm{Sr}_{2-x} \mathrm{La}_{x} \mathrm{Cu} \mathrm{O}_{6+\delta}$. Science, 343:390-392, 2014.

[20] E. H. da Silva Neto, P. Aynajian, A. Frano, R. Comin, E. Schierle, E. Weschke, A. Gyenis, J. Wen, J. Schneeloch, Z. Xu, S. Ono, G. Gu, M. Tacon, and A. Yazdani. Ubiquitous interplay between charge ordering and high-temperature superconductivity in cuprates. Science, 343:393-396, 2014.

[21] M. Hashimoto, G. Ghiringhelli, W. S. Lee, G. Dellea, A. Amorese, C. Mazzoli, K. Kummer, N. B. Brookes, B. Moritz, Y. Yoshida, H. Eisaki, Z. Hussain, T. P. Devereaux, Z. X. Shen, and L. Braicovich. Direct observation of bulk charge modulations in optimally doped $\mathrm{Bi}_{1.5} \mathrm{~Pb}_{0.6} \mathrm{Sr}_{1.54} \mathrm{CaCu}_{2} \mathrm{O}_{8+\delta}$. Phys. Rev. B, 89:220511(R), 2014.

[22] S. Blanco-Canosa, A. Frano, E. Schierle, J. Porras, T. Loew, M. Minola, M. Bluschke, E. Weschke, B. Keimer, and M. Le Tacon. Resonant X-ray scat- 
tering study of charge-density wave correlations in $\mathrm{YBa}_{2} \mathrm{Cu}_{3} \mathrm{O}_{6+x}$. Phys. Rev. B, 90:054513, 2014.

[23] E. H. da Silva Neto, R. Comin, F. He, R. Sutarto, Y. Jiang, R. L. Greene, G. A. Sawatzky, and A. Damascelli. Charge ordering in the electron-doped superconductor $\mathrm{Nd}_{2-x} \mathrm{Ce}_{x} \mathrm{CuO}$. Science, 347:282-285, 2015.

[24] W. D. Wise, M. C. Boyer, K. Chatterjee, , T. Kondo, T. Takeuchi, H. Ikuta, Yayu Wang, and E. W. Hudson. Charge-density-wave origin of cuprate checkerboard visualized by scanning tunnelling microscopy. Nature Phys., 4:696-699, 2008.

[25] T. Hanaguri, Y. Kohsaka, J. C. Davis, C. Lupien, I. Yamada, M. Azuma, M. Takano, K. Ohishi, M. Ono, and H. Takagi. Quasiparticle interference and superconducting gap in $\mathrm{Ca}_{2-x} \mathrm{Na}_{x} \mathrm{CuO}_{2} \mathrm{Cl}_{2}$. Nature Phys., 3:865-871, 2007.

[26] R. Comin, R. Sutarto, F. He, E. H. da Silva Neto, L. Chauviere, A. Frano, R. Liang, W. N. Hardy, D. A. Bonn, Y. Yoshida, H. Eisaki, A. J. Achkar, D. G. Hawthorn, B. Keimer, G. A. Sawatzky, and A. Damascelli. Symmetry of charge order in cuprates. Nature Materials, 14:796-800, 2015.

[27] A. J. Achkar, F. He, R. Sutarto, C. McMahon, M. Zwiebler, M. Hücher, G. D. Gu, R. Liang, D. A. Bonn, W. N. Hardy, J. Geck, and D. G. Hawthorn. Orbital symmetry of charge-density-wave order in $\mathrm{La}_{1.875} \mathrm{Ba}_{0.125} \mathrm{CuO}$ and $\mathrm{YBa}_{2} \mathrm{Cu}_{3} \mathrm{O}_{6.67}$. Nature Materials, 15:616-620, 2016.

[28] K. Fujita, M. H. Hamidian, S. D. Edkins, C. K. Kim, Y. Kohsaka, M. Azuma, M. Takano, H. Takagi, H. Eisaki, S. Uchida, A. Allais, M. J. Lawler, E. A. Kim, S. Sachdev, and J. C. Davis. Direct phase-sensitive identification of a $d$-form factor density wave in underdoped cuprates. PNAS, 111:E3026-E3032, 2014.

[29] K. Fujita, C. K. Kim, I. Lee, J. Lee, M. H. Hamidian, I. A. Firmo, S. Mukhopadhyay, H. Eisaki, S. Uchida, M. J. Lawler, E. A. Kim, and J. C. Davis. Simultaneous transitions in cuprate momentum-space topology and electronic symmetry breaking. Science, 344:612-616, 2014. 
[30] M. Hashimoto, I. Vishik, R. He, T. Devereaux, and Z. Shen. Energy gaps in hightransition-temperature cuprate superconductors. Nature Phys., 10:483-495, 2014.

[31] F. C. Zhang and T.M. Rice. Effective Hamiltonian for the superconducting $\mathrm{Cu}$ oxides. Phys. Rev. B, 37:3759-3761, 1988.

[32] A. Allais, J. Bauer, and S. Sachdev. Bond order instabilities in a correlated twodimensional metal. Phys. Rev. B, 90:155114, 2014.

[33] M. Metlitski and S. Sachdev. Instabilities near the onset of spin density wave order in metals. New J. Phys., 12:105007, 2010.

[34] M. Metlitski and S. Sachdev. Quantum phase transitions of metals in two spatial dimensions: Ii. Spin density wave order. Phys. Rev. B, 82:075128, 2010.

[35] R. B. Laughlin. Hartree-Fock computation of the high- $T_{c}$ cuprate phase diagram. Phys. Rev. B, 89:035134, 2014.

[36] E. Fradkin, S. Kivelson, and J. Tranquada. Colloquium: Theory of intertwined orders in high temperature superconductors. Rev. Mod. Phys., 87:457-482, 2015.

[37] E. Berg, E. Fradkin, S. Kivelson, and J. Tranquada. Striped superconductors: how spin, charge and superconducting orders intertwine in the cuprates. New J. Phys., $11: 115004,2009$.

[38] F. Loder, S. Graser, A. Kampf, and T. Kopp. Mean-field pairing theory for the charge-stripe phase of high-temperature cuprate superconductors. Phys. Rev. Lett., 107:187001, 2011.

[39] P. A. Lee. Amperean pairing and the pseudogap phase of cuprate superconductors. Phys. Rev. X, 4:031017, 2014.

[40] K. Yang, W. Chen, T. M. Rice, M. Sigrist, and F. C. Zhang. Nature of stripes in the generalized $t-J$ model applied to the cuprate superconductors. New J. Phys., 11:055053, 2009. 
[41] A. Himeda, T. Kato, and M. Ogata. Stripe states with spatially oscillating $d$ wave superconductivity in the two-dimensional $t-t^{\prime}-J$ model. Phys. Rev Lett., $88: 117001,2002$.

[42] C. P. Chou and T. K. Lee. Mechanism of formation of half-doped stripes in underdoped cuprates. Phys. Rev. B, 81:060503, 2010.

[43] C. P. Chou, N. Fukushima, and T. K. Lee. Cluster-glass wave function in the twodimensional extended $t-J$ model. Phys. Rev. B, 78:134530, 2008.

[44] A. Himeda and M. Ogata. Coexistence of $d_{x^{2}-y^{2}}$ superconductivity and antiferromagnetism in the two-dimensional $t-J$ model and numerical estimation of Gutzwiller factors. Phys. Rev B, 60:R9935-R9938, 1999.

[45] M. Ogata and A. Himeda. Superconductivity and antiferromagnetism in an extended gutzwiller approximation for $t-J$ model: effect of double-occupancy exclusion. J. Phys. Soc. Japan, 72:374-391, 2003.

[46] R. B. Christensen, P. J. Hirschfeld, and B. M. Anderson. Two routes to magnetic order by disorder in underdoped cuprates. Phys. Rev. B, 84:184511, 2011.

[47] C. P. Chou and T. K. Lee. Inhomogeneous state of the extended $t-J$ model on a square lattice: A variational Monte Carlo and Gutzwiller approximation study. Phys. Rev. B, 85:104511, 2012.

[48] D. Poilblanc. Stability of inhomogeneous superstructures from renormalized meanfield theory of the $t-J$ model. Phys. Rev. B, 72:060508, 2005.

[49] S. White and D. J. Scalapino. Density matrix renormalization group study of the striped phase in the $2 \mathrm{~d} t-J$ model. Phys. Rev. Lett., 80:1272-1275, 1998.

[50] S. White and D. J. Scalapino. Pairing on striped $t-t^{\prime}-J$ lattices. Phys. Rev. B, 79:220504, 2009.

[51] S. Sachdev and R. La Placa. Bond order in two-dimensional metals with antiferromagnetic exchange interactions. Phys. Rev. Lett., 111:027202, 2013. 
[52] J. C. Davis and D. H. Lee. Concepts relating magnetic interactions, intertwined electronic orders, and strongly correlated superconductivity. PNAS, 110:1762317630, 2013.

[53] Y. Wang and A. Chubukov. Charge-density-wave order with momentum $(2 q, 0)$ and $(0,2 q)$ within the spin-fermion model: Continuous and discrete symmetry breaking, preemptive composite order, and relation to pseudogap in hole-doped cuprates. Phys. Rev. B, 90:035149, 2014.

[54] P. Corboz, T. M. Rice, and M. Troyer. Competing states in the $t-J$ model: uniform $d$-wave state versus stripe state. Phys. Rev. Lett., 113:046402, 2014.

[55] B. X. Zheng, C. M. Chung, P. Corboz, G. Ehlers, M. P. Qin, R. M. Noark, H .Shi, S. R. White, S. Zhang, and G. K. Lin. Stripe order in the underdoped region of the two-dimensional Hubbard model. Science, 358:1155, 2017.

[56] M. Gutzwiller. Effect of correlation on the ferromagnetism of transition metals. Phys. Rev. Lett., 10:159-162, 1963.

[57] F. C. Zhang, C. Gros, T. M. Rice, and H. Shiba. A renormalized Hamiltonian approach to a resonant valence bond wavefunction. Supercond. Sci. Technol., 1:3646, 1988.

[58] M. Capello, M. Raczkowski, and D. Poilblanc. Stability of RVB hole stripes in high-temperature superconductors. Phys. Rev. B, 77:224502, 2008.

[59] M. H. Hamidian, S. D. Edkins, S. H. Joo, A. Kostin, H. Eisaki, S. Uchida, M. J. Lawler, E. A. Kim, A. P. Mackenzie, K. Fujita nad J. Lee, and J. C. Davis. Detection of a Cooper-pair density wave in $\mathrm{Ba}_{2} \mathrm{Sr}_{2} \mathrm{CaCu}_{2} \mathrm{O}_{8+x}$. Nature, 532:343, 2016.

[60] M. Vojta. Lattice symmetry breaking in cuprate superconductors: Stripes, nematics, and superconductivity. Adv. Phys., 58:699-820, 2009.

[61] B. Keimer, S. A. Kivelson, M. R. Norman, S. Uchida, and J. Zaanen. From quantum matter to superconductivity in copper oxides. Nature, 518:179-186, 2015. 
[62] A. Shekhter, B. J. Ramshaw, R. Liang, W. N. Hardy, D. A. Bonn, F. F. Balakirev, R. D. McDonald, J. B. Betts, S. C. Riggs, and A. Migliori. Bounding the pseudogap with a line of phase transitions in $\mathrm{YBa}_{2} \mathrm{Cu}_{3} \mathrm{O}_{6+\delta}$. Nature, 498:75-77, 2013.

[63] L. Zhao, C. A. Belvin, R. Liang anf D. A. Bonn, W. N. Hardy, N. P. Armitage, and D. Hsieh. A global inversion-symmetry-broken phase inside the pseudogap region of $\mathrm{YBa}_{2} \mathrm{Cu}_{3} \mathrm{O}_{y}$. Nature Phys., 13:250-254, 2017.

[64] P. Bourges and Y. Sidis. Novel magnetic order in the pseudogap state of high- $T_{c}$ copper oxides superconductors. Comptes Rendus Physique, 12:461-479, 2011.

[65] Y. Sato, S. Kasahara, H. Murayama, Y. Kasahara, E. G. Moon, T. Nishizaki, T. Loew, J. Porras, B. Keimer, T. Shibauchi, and Y. Matsuda. Thermodynamic evidence for a nematic phase transition at the onset of the pseudogap in $\mathrm{YBa}_{2} \mathrm{Cu}_{3} \mathrm{O}_{y}$. Nature Phys., 13:1074-1078, 2017.

[66] R. Comin, R. Sutarto, E. H. da Silva Neto, L. Chauviere, R. Liang, Hardy W, N, D. A. Bonn, F. He, G. A. Sawatzky, and A. Damascelli. Broken translational and rotational symmetry via charge stripe order in underdoped $\mathrm{YBa}_{2} \mathrm{Cu}_{3} \mathrm{O}_{6+y}$. Science, 347:1335-1339, 2015.

[67] J. Wu, A. T. Bollinger, X. He, and I. Bo žović. Spontaneous breaking of rotational symmetry in copper oxide superconductors. Nature, 547:432-435, 2017.

[68] R. Comin and A. Damascelli. Resonant X-ray scattering studies of charge order in cuprates. Ann. Rev. Condensed Matter Phys., 7:369-405, 2016.

[69] A. Yazdani, E. H. da Silva Neto, and P. Aynajian. Spectroscopic imaging of strongly correlated electronic states. Ann. Rev. Condensed Matter Phys., 7:11-33, 2016.

[70] D. H. Torchinsky, F. Mahmood, A. T. Bollinger, I. Božović, and N. Gedik. Fluctuating charge-density waves in a cuprate superconductor. Nature Materials, 12:387$391,2013$. 
[71] J. R. Kirtley, C. C. Tsuei, A. Ariando, C. J. M. Verwijs, S. Harkema, and J. W. M. Hilgenkamp. Angle-resolved phase-sensitive determination of the in-plane gap symmetry in $\mathrm{YBa}_{2} \mathrm{Cu}_{3} \mathrm{O}_{7-\delta}$. Nature Phys., 2:190-194, 2006.

[72] C. C. Tsuei, J. R. Kirtley, G. Hammerl, J. Mannhart, and H. Raffy abd Z. Z. Li. Robust $d_{x^{2}-y^{2}}$ pairing symmetry in hole-doped cuprate superconductors. Phys. Rev. Lett., 93:187004, 2004.

[73] W. W. Warren Jr., R. E. Walstedt, G. F. Brennert, R. J. Cava, R. Tycko, R. F. Bell, and G. Dabbagh. Cu spin dynamics and superconducting precursor effects in planes above Tc in $\mathrm{YBa}_{2} \mathrm{Cu}_{3} \mathrm{O}_{6.7}$. Phys. Rev. Lett., 62:1193-1196, 1989.

[74] T. Timusk and B. Statt. The pseudogap in high-temperature superconductors: An experimental survey. Rep. Prog. Phys., 62:61-122, 1999.

[75] D. S. Marshall, D. S. Dessau, A. G. Loeser, C. H. Park, A. Y. Matsuura, J. N. Eckstein, I. Bozovic, P. Fournier, A. Kapitulnik, W. E. Spicer, and Z. X. Shen. Unconventional electronic structure evolution with hole doping in $\mathrm{Bi}_{2} \mathrm{Sr}_{2} \mathrm{CaCu}_{2} \mathrm{O}_{8+\delta}$ : Angle-resolved photoemmision results. Phys. Rev. Lett., 76:4841-4844, 1996.

[76] A. G. Loeser, Z. X. Shen, D. S. Dessau, D. S. Marshall, C. H. Park, P. Fournier, and A. Kapitulnik. Excitation gap in the normal state of underdoped $\mathrm{Bi}_{2} \mathrm{Sr}_{2} \mathrm{CaCu}_{2} \mathrm{O}_{8+\delta}$. Science, 273:325-329, 1996.

[77] H. Ding, T. Yokoya, J. C. Campuzano, T. Takahashi, M. Randeria, M. R. Norman, T. Mochiku, K. Kadowaki, and J. Giapintzakis. Spectroscopic evidence for a pseudogap in the normal state of underdoped high- $T_{c}$ superconductors. Nature, 382:51-54, 1996.

[78] A. Kanigel, M. R. Norman, M. Randeria, U. Chatterjee, S. Souma, A. Kaminski, H. M. Fretwell, S. Rosenkranz, M. Shi, T. Sato, T. Takahashi, Z. Z. Li, H. Raffy, K. Kadowaki, D. Hinks, L. Ozyuzer, and J. C. Campuzano. Evolution of the pseudogap from Fermi arcs to the nodal liquid. Nature Phys., 2:447-451, 2006. 
[79] K. Nakayama, T. Sato, Y. Sekiba, K. Terashima, P. Richard, T. Takahashi, K. Kudo, N. Okumura, T. Sasaki, and N. Kobayashi. Evolution of a pairing-induced pseudogap from the superconducting gap of $(\mathrm{Bi}, \mathrm{Pb})_{2} \mathrm{Sr}_{2} \mathrm{CuO}_{6}$. Phys. Rev. Lett., 102:227006, 2009.

[80] N. Doiron-Leyraud, C. Proust, D. LeBoeuf, J. Levallois, J. B. Bonnemaison, R. Liang, D. A. Bonn, W. N. Hardy, and L. Taillefer. Quantum oscillations and the Fermi surface in an underdoped high- $T_{c}$ superconductor. Nature, 447:565-568, 2007.

[81] A. F. Bangura, J. D. Fletcher, A. Carrington, J. Levallois, M. Nardone, B. Vignolle, P. J. Heard, N. Doiron-Leyraud, D. LeBoeuf, L. Taillefer, S. Adachi, C. Proust, and N. E. Hussey. Small fermi surface pockets in underdoped high temperature superconductors: Observation of Shubnikov-de Haas oscillations in $\mathrm{YBa}_{2} \mathrm{Cu}_{4} \mathrm{O}_{8}$. Phys. Rev. Lett., 100:047004, 2008.

[82] R. H. He, M. Hashimoto, H. Karapetyan, J. D. Koralek, J. P. Hinton, J. P. Testaud, V. Nathan, Y. Yoshida, H. Yao, K. Tanaka, W. Meevasana, R. G. Moore, D. H. Lu, S. K. Mo, M. Ishikado, H. Eisaki, Z. Hussain, T. P. Devereaux, S. A. Kivelson, J. Orenstein, A. Kapitulnik, and Z. X. Shen. From a single-band metal to a hightemperature superconductor via two thermal phase transitions. Science, 331:1579$1583,2011$.

[83] S. Huefner, M. A. Hossain, A. Damascelli, and G. A. Sawatzky. Two gaps make a high-temperature superconductor? Rep. Prog. Phys., 71:062501, 2008.

[84] P. A. Lee, N. Nagaosa, and X. G. Wen. Doping a Mott insulator: Physics of hightemperature superconductivity. Rev. Mod. Phys., 78:17, 2006.

[85] A. I. Larkin and Y. N. Ovchinnikov. Nonuniform state of superconductors. Sov. Phys.-JETP, 20:762, 1965.

[86] P. Fulde and R. A. Ferrell. Superconductivity in a strong spin-exchange field. Phys. Rev., 135:A550, 1964. 
[87] E. Berg, E. Fradkin, and S. A. Kivelson. Theory of the striped superconductor. Phys. Rev. B, 79:064515, 2009.

[88] E. Berg, E. Fradkin, and S. A. Kivelson. Charge-4e superconductivity from pairdensity-wave order in certain high-temperature superconductors. Nature Phys., 5:830-833, 2009.

[89] D. Podolsky, E. Demler, K. Damle, and B. I. Halperin. Translational symmetry breaking in the superconducting state of the cuprates: Analysis of the quasiparticle density of states. Phys. Rev. B, 67:094514, 2003.

[90] H. D. Chen, O. Vafek, A. Yazdani, and S. C. Zhang. Pair density wave in the pseudogap state of high temperature superconductors. Phys. Rev. Lett., 93:187002, 2004.

[91] K. B. Efetov and C. Pépin. Pseudogap state near a quantum critical point. Nature Phys., 9:442-446, 2013.

[92] Y. Wang, D. F. Agterberg, and A. Chubukov. Coexistence of charge-density-wave and pair-density-wave orders in underdoped cuprates. Phys. Rev. Lett., 114:197001, 2015.

[93] E. Berg, E. Fradkin, E. A. Kim, S. A. Kivelson, V. Oganesyan, J. M. Tranquada, and S. C. Zhang. Dynamical layer decoupling in a stripe-ordered high- $T_{c}$ superconductor. Phys. Rev. Lett., 99:127003, 2007.

[94] D. Agterberg and H. Tsunetsugu. Dislocations and vortices in pair-density-wave superconductors. Nature Phys., 4:639-642, 2008.

[95] S. Baruch and D. Orgad. Spectral signatures of modulated $d$-wave superconducting phases. Phys. Rev. B, 77:174502, 2008.

[96] D. R. Hofstadter. Energy levels and wave functions of Bloch electrons in rational and irrational magnetic fields. Phys. Rev. B, 14:2239, 1976. 
[97] M. Aidelsburger, M. Atala, M. Lohse, J. T. Barreiro, B. Paredes, and I. Bloch. Realization of the Hofstadter Hamiltonian with ultracold atoms in optical lattices. Phys. Rev. Lett., 111:185301, 2013.

[98] H. Miyake, G. A. Siviloglou, C. J. Kennedy, W. C. Burton, and W. Ketterle. Realizing the Harper Hamiltonian with laser-assisted tunneling in optical lattices. Phys. Rev. Lett., 111:185302, 2013.

[99] C. J. Kennedy, W. C. Burton, W. C. Chung, and W. Ketterle. Observation of BoseEinstein condensation in a strong synthetic magnetic field. Nature Phys., 11:859$864,2015$.

[100] M. Aidelsburger, M. Lohse, C. Schweizer, M. Atala, J. T. Barreiro, S. Nascimbène, N. R. Cooper, I. Bloch, and N. Goldman. Measuring the Chern number of Hofstadter bands with ultracold bosonic atoms. Nature Phys., 11:162-166, 2015.

[101] M. Mancini, G. Pagano, G. Cappellini, L. Livi, M. Rider, J. Catani, C. Sias, P. Zoller, M. Inguscio, M. Dalmonte, and L. Fallani. Observation of chiral edge states with neutral fermions in synthetic Hall ribbons. Science, 349:1510-1513, 2015.

[102] B. K. Stuhl, H. I. Lu, L. M. Aycock, D. Genkina, and I. B. Spielman. Visualizing edge states with an atomic Bose gas in the quantum Hall regime. Science, 349:1514-1518, 2015.

[103] N. R. Cooper and J. Dalibard. Reaching fractional quantum Hall states with optical flux lattices. Phys. Rev. Lett., 110:185301, 2013.

[104] N. Goldman, J. C. Budich, and P. Zoller. Topological quantum matter with ultracold gases in optical lattices. Nature Physics, 12:639-645, 2016.

[105] M. Lacki, H. Pichler, A. Sterdyniak, A. Lyras, V. E. Lembessis, O. Al-Dossary, J. C. Budich, and P. Zoller. Quantum Hall physics with cold atoms in cylindrical optical lattices. Phys. Rev. A, 93:013604, 2016. 
[106] T. H. Hansson, M. Hermanns, S. H. Simon, and S. F. Viefers. Quantum Hall physics: Hierarchies and conformal field theory techniques. Rev. Mod. Phys., 89:025005, 2017.

[107] A. S. Sørensen, E. Demler, and M. D. Lukin. Fractional quantum Hall states of atoms in optical lattices. Phys. Rev. Lett., 94:086803, 2005.

[108] M. Hafezi, A. S. Sørensen, E. Demler, and M. D. Lukin. Fractional quantum Hall effect in optical lattices. Phys. Rev. A, 76:023613, 2007.

[109] G. Möller and N. R. Cooper. Composite fermion theory for bosonic quantum Hall states on lattices. Phys. Rev. Lett., 103:105303, 2009.

[110] A. Sterdyniak, N. Regnault, and G. Möller. Particle entanglement spectra for quantum Hall states on lattices. Phys. Rev. B, 86:165314, 2012.

[111] I. Affleck and J. B. Marston. Large-n limit of the Heisenberg-Hubbard model: Implications for high- $T_{c}$ superconductors. Phys. Rev. B, 37:R3774, 1988.

[112] J. B. Marston and I. Affleck. Large- $n$ limit of the Hubbard-Heisenberg model. Phys. Rev. B, 39:11538, 1989.

[113] G. Kotliar. Resonating valence bonds and $d$-wave superconductivity. Phys. Rev. B, $37: 3664,1988$.

[114] P. Lederer, D. Poilblanc, and T. M. Rice. Superconductivity from commensurate flux phases. Phys. Rev. Lett., 63:1519, 1989.

[115] F. Nori, E. Abrahams, and G. T. Zimanyi. Generalized flux states of the $t-J$ model. Phys. Rev. B, 41:R7277, 1990.

[116] M. Gerster, M. Rizzi, P. Silvi, M. Dalmonte, and S. Montangero. Fractional quantum Hall effect in the interacting Hofstadter model via tensor networks. Phys. Rev. B, 96:195123, 2017. 
[117] D. Poilblanc, Y. Hasegawa, and T. M. Rice. Numerical study of flux phases in the $t-J$ model. Phys. Rev. B, 41:1949, 1990.

[118] E. Ercolessi, P. Pieri, and M. Roncaglia. Phase separation and three-site hopping in the 2-dimensional $t-J$ model. Phys. Lett. A, 233:451, 1997.

[119] P. Choubey, T. Berlijn, A. Kreisel, C. Cao, and P. J. Hirschfeld. Visualization of atomic-scale phenomena in superconductors: Application to FeSe. Phys. Rev. B, 90:134520, 2014.

[120] A. A. Mostofi, J. R. Yates, G. Pizzi, Y. S. Lee, I. Souza, D. Vanderbilt, and N. Marzari. An updated version of wannier90: A tool for obtaining maximallylocalised Wannier functions. Comput. Phys. Commun., 185:2309-2310, 2014.

[121] A. Kreisel, P. Choubey, T. Berlijn, W. Ku, B. M. Anderson, and P. J. Hirschfeld. Interpretation of scanning tunneling quasiparticle interference and impurity states in cuprates. Phys. Rev. Lett., 114:217002, 2015.

[122] M. Schmid, B. M. Anderson, A. P. Kampf, and P. J. Hirschfeld. d-wave superconductor as a catalyst for antiferromagnetism in underdoped cuprates. New J. Phys., 12:053043, 2010.

[123] D. Green, L. Santos, and C. Chamon. Isolated flat bands and spin-1 conical bands in two-dimensional lattices. Phys. Rev. B, 82:075104, 2010.

[124] W. L.. McMillan. Theory of discommensurations and the commensurateincommensurate charge-density-wave phase transition. Phys. Rev. B, 14:1469$1502,1976$.

[125] A. Mesaros. Commensurate $4 a_{0}$-period charge density modulations throughout the $\mathrm{Bi}_{2} \mathrm{Sr}_{2} \mathrm{CaCu}_{2} \mathrm{O}_{8+x}$ pseudogap regime. PNAS, 113:12661, 2016.

[126] S. A. Kivelson, I. P. Bindloss, E. Fradkin, V. Oganesyan, J. M. Tranquada, A. Kapitulnik, and C. Howald. How to detect fluctuating stripes in the high-temperature superconductors. Rev. Mod. Phys., 75:1201, 2003. 
[127] H. Jang, W. S. Lee, H. Nojiri, S. Matsuzawa, H. Yasumura, L. Nie, A. V. Maharaj, S. Gerber, Y. J. Liu, A. Mehta, D. A. Bonn, R. Liang, W. N. Hardy, C. A. Burns, Z. Islam, S. Song, J. Hastings, T. P. Devereaux, Z. X. Shen, S. A. Kivelson, C. C. Kao, D. Zhu, and J. S. Lee. Three-dimensional charge density wave order in $\mathrm{YBa}_{2} \mathrm{Cu}_{3} \mathrm{O}_{6.67}$ at high magnetic fields. Science, 350:949-952, 2015.

[128] H. Jang, W. S. Lee, H. Nojiri, S. Matsuzawa, H. Yasumura, L. Nie, A. V. Maharaj, S. Gerber, Y. J. Liu, A. Mehta, D. A. Bonn, R. Liang, W. N. Hardy, C. A. Burns, Z. Islam, S. Song, J. Hastings, T. P. Devereaux, Z. X. Shen, S. A. Kivelson, C. C. Kao, D. Zhu, and J. S. Lee. Ideal charge-density-wave order in the high-field state of superconducting ybco. PNAS, 113:14645-14650, 2016.

[129] E. M. Forgan, E. Blackburn, A. T. Holmes, A. K. R. Briffa, J. Chang, L. Bouchenoire, S. D. Brown, R. Liang, D. Bonn, W. N. Hardy, N. B. Christensen, M. V. Zimmermann, M. Hucker, and S. M. Hayden. The microscopic structure of charge density waves in underdoped $\mathrm{YBa}_{2} \mathrm{Cu}_{3} \mathrm{O}_{6.54}$ revealed by X-ray diffraction. Nature Comm., 6:10064, 2015.

[130] I. M. Vishik, M. Hashimoto, R. H. He, W. S. Lee, F. Schmitt, D. Lu, R. G. Moore, C. Zhang, W. Meevasana, T. Sasagawa, S. Uchida, K. Fujita, S. Ishida, M. Ishikado, Y. Yoshida, H. Eisaki, Z. Hussain, T. P. Devereaux, and Z. X. Shen. Phase competition in trisected superconducting dome. PNAS, 109:18332-18337, 2012.

[131] K. Y. Yang, C. T. Shih, C. P. Chou, S. M. Huang, T. K. Lee, and F. C. Zhang. Lowenergy physical properties of high- $T_{c}$ superconducting $\mathrm{Cu}$ oxides: A comparison between the resonating valence bond and experiments. Phys. Rev. B, 73:224513, 2006.

[132] E. W. Huang, C. B. Mendl, S. Liu, S. Johnson, H. C. Jiang, B. Moritz, and T. P. Devereaux. Numerical evidence of fluctuating stripes in the normal state of high$T_{c}$ cuprate superconductors. Science, 358:1161-1164, 2017. 
[133] Z. A. Xu, N. P. Ong, Y. Wang, and T. Kakeshita nad S. Uchida. Vortex-like excitations and the onset of superconducting phase fluctuation in underdoped $\mathrm{La}_{2-x} \mathrm{Sr}_{x} \mathrm{CuO}$. Nature, 406:486-488, 2000.

[134] P. W. Anderson. Last words on the cuprates. arXiv, 1612:03919, 2016.

[135] J. Chang, E. Blackburn, A. T. Holmes, N. B. Christensne, J. Larsen, J. Mesot, R. Liang, D. A. Bonn, W. N. Hardy, A. Watenphul, M. V. Zimmermann, E. M. Forgen, and S. M. Hayden. Direct observation of competition between superconductivity and charge density wave order in $\mathrm{YBa}_{2} \mathrm{Cu}_{3} \mathrm{O}_{6.67}$. Nature Phys., 8:871$876,2012$.

[136] T. Wu, H. Mayaffre, S. Kramer, M. Horvatic, C. Berthier, P. L. Kuhns, A. P. Reyes, R. Liang, W. N. Hardy, D. A. Bonn, and M. H. Julien. Emergence of charge order from the vortex state of a high-temperature superconductor. Nature Comm., 4:2113, 2013.

[137] S. Blanco-Canosa nad A. Frano, T. Loew, Y. Lu, J. Porras, G. Ghiringhelli, M. Minola, C. Mazzoli, L. Braicovich, E. Schierle, E. Weschke, M. Le Tacon, and B. Keimer. Momentum-dependent charge correlations in $\mathrm{YBa}_{2} \mathrm{Cu}_{3} \mathrm{O}_{6+\delta}$ superconductors probed by resonant X-ray scattering: Evidence for three competing phases. Phys. Rev. Lett., 110:187001, 2013.

[138] S. D. Edkins, A. Kostin, K. Fujita, A. P. Mackenzie, H. Eisaki, S. Uchida nad S. Sachdev, M. J. Lawler, E. A. Kim, J. C. Davis, and M. H. Hamidian. Magneticfield induced pair density wave state in the cuprate vortex halo. arXiv, 1802:04673, 2018.

[139] S. Kawasaki, Z. Li, M. Kitahashi, C. T. Lin, P. L. Kuhns, A. P. Reyes, and G. Zheng. Charge-density-wave order takes over antiferromagnetism in $\mathrm{Bi}_{2} \mathrm{Sr}_{2-x} \mathrm{La}_{x} \mathrm{CuO} \mathrm{O}_{6}$ superconductors. Nature Comm., 8:1267, 2017. 
[140] P. W. Anderson, B. S. Shastry, and D. Hristopulos. Class of variational singlet wave functions for the hubbard model away from half filling. Phys. Rev. B, 40:8939, 1989.

[141] X. G. Wen, F. Wilczek, and A. Zee. Chiral spin states and superconductivity. Phys. Rev. B, 39:11413-11423, 1989.

[142] A. Wietek, A. Sterdyniak, and A. M. Läuchli. Nature of chiral spin liquids on the kagome lattice. Phys. Rev. B, 92:125122, 2015.

[143] A. Wietek and A. M. Läuchli. Chiral spin liquid and quantum criticality in extended $s=\frac{1}{2}$ heisenberg models on the triangular lattice. Phys. Rev. B, 95:035141, 2017.

[144] X. G. Wen and Q Niu. Ground-state degeneracy of the fractional quantum hall states in the presence of a random potential and on high-genus riemann surfaces. Phys. Rev. B, 41:9377, 1990.

[145] M. Claassen, C. H. Lee, R. Thomale, X. L. Qi, and T. P. Devereaux. Positionmomentum duality and fractional quantum hall effect in chern insulators. Phys. Rev. Lett., 114:236802, 2015.

[146] D. J. Thouless, M. Kohmoto, M. P. Nightingale, and M. denNijs. Quantized hall conductance in a two-dimensional periodic potential. Phys. Rev. Lett., 49:405, 1982.

[147] Q. Niu, D. J. Thouless, and Y.-S. Wu. Quantized hall conductance as a topological invariant. Phys. Rev. B, 31:3372, 1985.

[148] Didier Poilblanc. Twisted boundary conditions in cluster calculations of the optical conductivity in two-dimensional lattice models. Phys. Rev. B, 44:9562-9581, Nov 1991.

[149] T. Fukui, Y. Hatsugai, and H. Suzuki. Chern numbers in discretized brillouin zone: Efficient method of computing (spin) hall conductances. J. Phys. Soc. Jpn., $74: 1674,2005$. 
[150] S. Badoux, W. Tabis, F. Laliberté, G. Grissonnanche, B. Vignolle, D. Vignolles, J. Beard, D. A. Bonn, W. N. Hardy, R. Liang, N. Doiron-Leyraud, L. Taillefer, and C. Proust. Change of carrier density at the pseudogap critical point of a cuprate superconductor. Nature, 531:210, 2016.

[151] T. Esslinger. Fermi-hubbard physics with atoms in an optical lattice. Ann. Rev. of Condens. Matter Phys., 1:129, 2010. 


\section{Appendix A}

\section{Exact Diagonalization}

\section{A.1 Model}

We study by Lanczos ED an instance of the model given by Eq. (6.1) with $\Phi=q / 16, q=$ $0, \cdots, 15$, for the parameter $t=1$ and $J=0.3$, on a $4 \times 4$ lattice with periodic boundary conditions (2-torus geometry). We make a choice of gauge in which the $A_{i j}$ take the values shown in Fig. A.1(a).

$H$ preserves the total number of particles per spin $n_{\mu}=\sum_{i, \mu} n_{i}$, which is therefore a good quantum number. For one, this allows us to treat sectors of different particle number. We will label them by the particle filling $\rho=\left(n_{\uparrow}+n_{\downarrow}\right) / 32$. On the other hand, the model is also invariant under global $S U(2)$ spin rotations. In particular, it is unaffected by global $U(1)$ rotations around the $z$-axis. The eigenvalue of the operator $S_{z}=\sum_{i}\left(S_{z}\right)_{i}$ is therefore a good quantum number, and we can diagonalize $H$ in each $S_{z}$ subspace separately. Finite-size precursors to ferromagnetic order can be inferred from degenerate energy eigenvalues at different $S_{z}$, where a multiplicity of $2 S+1$ corresponds to a spin polarization of magnitude $S$.

\section{A.2 Many-body Chern number}

To calculate the many-body Chern number, we introduce twisted boundary conditions [148] labeled by the twisting angles $\phi=\left(\phi_{x}, \phi_{y}\right)^{\mathrm{T}}$. This amounts to all many-body states $|\Psi\rangle$ 
a)

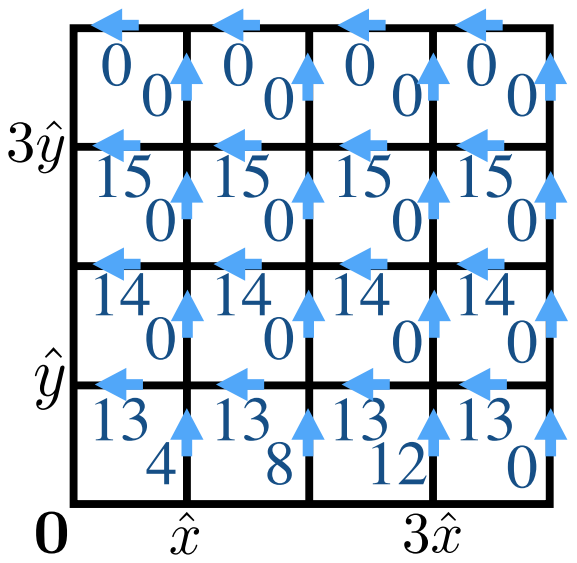

b)

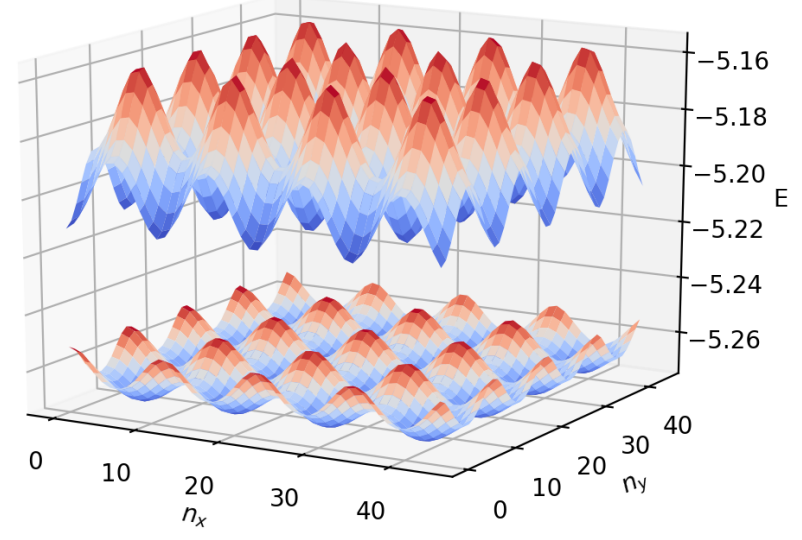

Figure A.1: (a) Vector potential gauge choice for $\Phi=q / 16, q=0, \cdots, 15$. Periodic boundary conditions are assumed. $A_{i j}$ in units of $F=2 \pi \Phi$ is given by the integer number shown between site $i$ and $j$, with positive sign if the respective arrow points from site $i$ to site $j$, and negative sign otherwise. (b) Spectrum $E(\phi)$ as a function of inserted flux for $\nu=1 / 5$. The Chern number evaluates to 6 , however, there is no indication for a topological GSD.

obeying

$$
T_{L \hat{x}}|\Psi\rangle=e^{\mathrm{i} \phi_{x}}|\Psi\rangle, \quad T_{L \hat{y}}|\Psi\rangle=e^{\mathrm{i} \phi_{y}}|\Psi\rangle
$$

where $T_{\boldsymbol{r}}$ is any operator translating a single particle by $\boldsymbol{r}$. In practice, this prescription can be implemented by making the substitutions

$$
\begin{aligned}
A_{i, i+\hat{x}} & \rightarrow A_{i, i+\hat{x}}+\phi_{x}, \\
\forall i & =(L-1) \hat{x}+n \hat{y}, \quad n=0, \cdots, L-1, \\
A_{i, i+\hat{y}} & \rightarrow A_{i, i+\hat{y}}+\phi_{y}, \\
\forall i & =(L-1) \hat{y}+n \hat{x}, \quad n=0, \cdots, L-1 .
\end{aligned}
$$

The Chern number of the $n$-th many body eigenstate $|n\rangle$ is then defined as [147]

$$
C=\frac{1}{2 \pi \mathrm{i}} \int_{0}^{2 \pi} d \phi_{x} \int_{0}^{2 \pi} d \phi_{y} \epsilon^{a b}\left\langle\partial_{a} n(\phi) \mid \partial_{b} n(\phi)\right\rangle
$$



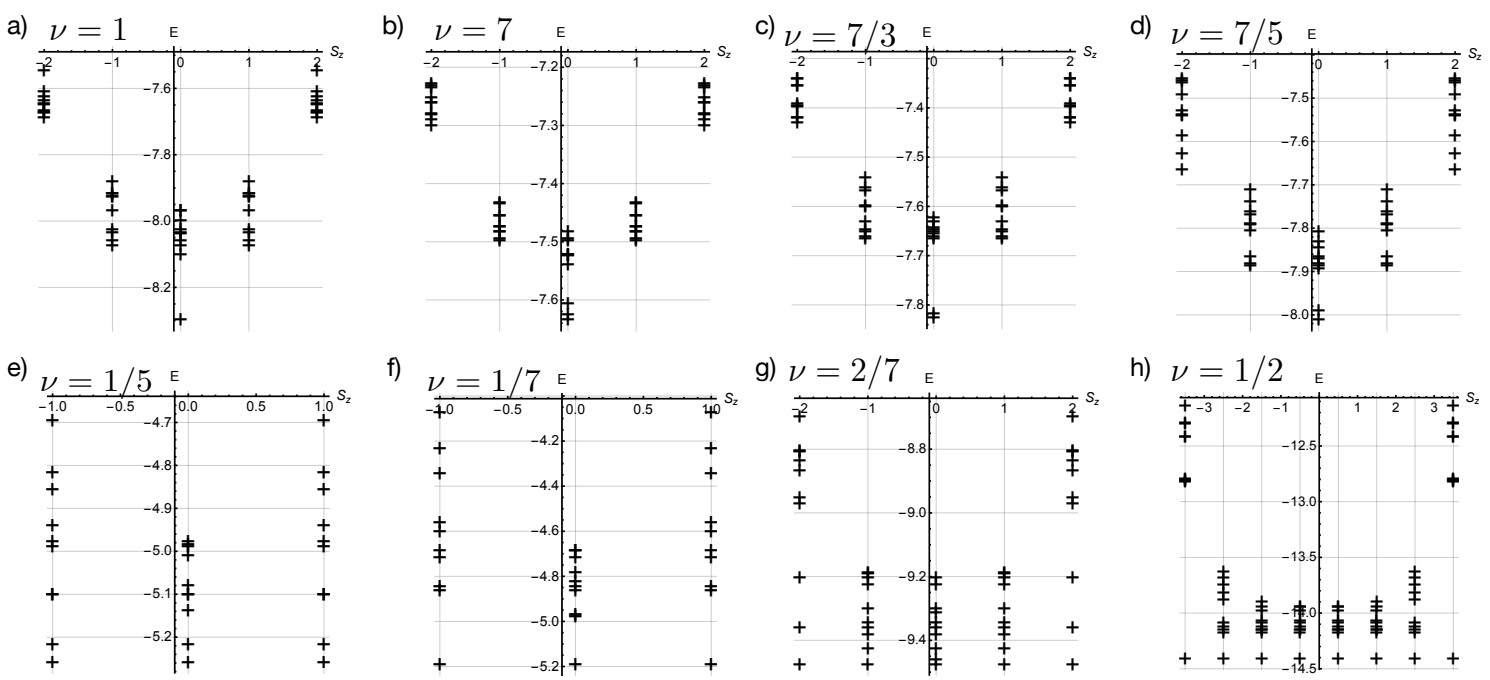

h) $\nu=1 / 2$

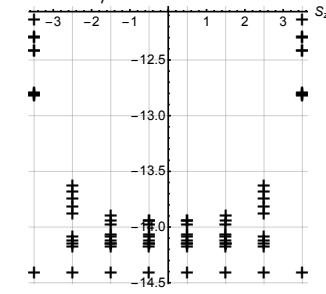

Figure A.2: Lanczos ED spectrum of $H$ for various values of $\nu$, with $\Phi$ and $\rho$ as given by Table A.1. When there is no magnetization, only the $S_{z}=0, \pm 1$ sector is shown.

where $\epsilon^{a b}, a, b=x, y$ is the totally antisymmetric $2 \times 2$ tensor, $\partial_{a}=\partial / \partial \phi_{a}$, and we assume that $|n(\phi)\rangle$ is non-degenerate at all $\phi$.

In practice, to calculate the Chern number via ED, we consider a lattice of twisted boundary conditions $\phi_{a}=2 \pi n_{a} / N, n_{a}=0 \ldots N-1$, and evaluate $C$ using the prescription of Ref. [149]. Here, we have chosen $N=45$ for the cases corresponding to low fermion densities. For the cases corresponding to $\rho=7 / 16$ filling, i.e., 2 holes on $4 \times 4$, which have a much larger Hilbert space, we have taken $N=10$ and checked the consistency of the results with $N=32$ in the special case where $\Phi=5 / 16$. See Fig. A.1(b) for an example of the dependence of the spectrum of $H$ on inserted flux.

\begin{tabular}{|c|c|c|c|c|c|c|c|}
\hline$\rho$ & $\Phi$ & $\nu / \nu^{*}$ & $S$ & $E_{0}$ & $E_{\text {kin }}$ & $E_{\text {pot }}$ & $C_{\mathrm{ED}}$ \\
\hline \hline $7 / 16$ & $7 / 16$ & 1 & 0 & -8.2901 & -6.39644 & -1.89369 & 2 \\
\hline $7 / 16$ & $5 / 16$ & $7 / 5$ & 0 & -8.0058 & -6.04586 & -1.95997 & 6 \\
\hline $7 / 16$ & $3 / 16$ & $7 / 3$ & 0 & -7.8204 & -5.90818 & -1.91226 & 6 \\
\hline $7 / 16$ & $1 / 16$ & 7 & 0 & -7.6298 & -5.73802 & -1.89179 & 14 \\
\hline $7 / 32$ & $7 / 16$ & $1^{*}$ & $7 / 2$ & -14.3874 & -14.7165 & 0.329042 & 1 \\
\hline $1 / 8$ & $1 / 4$ & $1^{*}$ & 2 & -11.2393 & -11.3132 & 0.0739077 & 1 \\
\hline $1 / 8$ & $7 / 16$ & $4 / 7^{*}$ & 2 & -9.4670 & -9.55201 & 0.0849988 & 4 \\
\hline $1 / 16$ & $5 / 16$ & $2 / 5^{*}$ & 1 & -5.2519 & -5.26527 & 0.0133967 & 6 \\
\hline $1 / 16$ & $7 / 16$ & $2 / 7^{*}$ & 1 & -5.1794 & -5.19852 & 0.0190752 & 2 \\
\hline
\end{tabular}

Table A.1: Summary of the Lanczos exact diagonalization results. 


\section{A.3 Results}

We diagonalize $H$ for various filling factors $\nu$, defined as $\nu \equiv \rho / \Phi$. The GS energies, as well as spin polarizations and Chern numbers are summarized here again in Table A.1. Figure A.2 furthermore shows the spectra for the $S_{z}$ values of interest. Taking $|0\rangle$ to be the many-body GS of $H$, we define $E=\langle 0|H| 0\rangle, E_{\text {kin }}=\left\langle 0\left|H_{\text {kin }}\right| 0\right\rangle$ and $E_{\text {pot }}=\left\langle 0\left|H_{\text {pot }}\right| 0\right\rangle$, with $H_{\text {kin }}$ and $H_{\text {pot }}$ given by Eq. (6.1). 


\section{Appendix B}

\section{Induced Topological-Trivial Transition}

When it comes to the topological transition, interestingly, it is possible to induce a transition from a topologically non-trivial phase to a trivial phase by adding a staggered potential to the Hamiltonian, as was implemented in a cold atom experiment [100]. The staggered potential of magnitude $\Gamma$ takes the form:

$$
H_{\text {staggered }}=\frac{\Gamma}{2} \sum_{i}\left[(-1)^{i_{x}}+(-1)^{i_{y}}\right] n_{i}
$$

where $n_{i}=c_{i}^{\dagger} c_{i}$ at lattice sites $i=\left(i_{x}, i_{y}\right)$. Notice that since we are considering fully polarized systems, we discard the spin index. Since the staggered potential has a $2 \times 2$ spatial periodicity, it will induce CDW modulation via linear response, which may prohibit the formation of a topological phase (associated to a non-zero Chern number). To match the experimental setup, we choose here $\rho=1 / 8$ and $\Phi=1 / 4$, which gives $\nu^{*}=2 \nu=1$. This corresponds to the scenario of a completely filled lowest Hofstadter band. The magnetic gauge used is shown in Fig. 6.1. Our aim is to investigate the role of the interaction namely, (i) whether it could induce a lattice instability involving spontaneous translation symmetry breaking and/or (ii) whether it will affect the location of the transition.

To investigate (i) we have used a $4 \times 4$ supercell, larger than the $2 \times 2$ magnetic unit cell, when solving the RMFT equations. In fact, no such instability was found, i.e., the $2 \times 2$ unit cell corresponds to the translation symmetry of the ground state.

We have considered different staggered potential strengths and observed the phase 
transition as a function of $\Gamma$ showing results very similar to the non interacting case [97, 100]. A qualitative understanding of the effect of the interaction on the location of the transition can be obtained as follows. The mean-field Hamiltonian is in fact identical to the non-interacting one up to renormalizations of the hopping term $\tilde{t} \approx t\left(1+\frac{J}{4} \chi\right)$ and of the magnitude of the $2 \times 2$ potential $\tilde{\Gamma}=\Gamma\left(1+J \chi_{\Gamma}\right)$, where the $J \chi_{\Gamma} \Gamma$ term originates from the induced effective local chemical potential (Eq. 6.10) whose spatial periodicity is (in linear response) identical to the one of the perturbation $\Gamma$, and $\chi_{\Gamma}$ is a susceptibility at momentum $(\pi / 2, \pi / 2)$. As shown in Ref.[100], the transition for a non-interacting system occurs at $\Gamma=2 t$, which for the mean-field Hamiltonian translates into $\tilde{\Gamma}=2 \tilde{t}$ providing a simple expression for the critical staggered strength $\Gamma^{*}$,

$$
\Gamma^{*}=2 t \frac{1+\frac{1}{4} J \chi}{1+J \chi_{\Gamma}} .
$$

Using the numerical values of $\chi$ (Eq. 6.9) and $\chi_{\Gamma}$ at $J=0.3$, we obtain $\Gamma^{*} \simeq 2.048 t$. This signifies that interactions increase the size of the trivial region only very slightly, which may be a generic feature.

Further details of the RMFT calculations of the Hofstadter $t-J$ model for $\rho=1 / 8$ and $\Phi=1 / 4$ and in the presence of a staggered potential are given next. We have considered different staggered strengths and observed the phase transition described in Ref. [100] as a function of $\Gamma$. In Fig. B.1, the band structures for four representative values of $\Gamma$ are shown within a reduced $\mathrm{BZ}, k_{x}, k_{y} \in[-\pi / 4, \pi / 4]$. Note that the modulations generated by a nonzero $\Gamma$ all have $2 \times 2$ periodicity, indicating that the bands connecting with each other at the zone boundary are in fact due to the (artificial) band folding originating from the larger supercell used in the RMFT calculation, and therefore should be considered as the same bands. In Fig. B.1(a), the bands are topologically trivial since their Chern numbers are zero. There is also an obvious band gap between the lowest and middle bands. As we lower the staggered value, the gap shrinks gradually and closes eventually at $\Gamma \simeq 2 t$. This is the point when the system enters the topologically non-trivial phase since now summing up the Chern numbers of the lowest and middle bands gives 1. As we further lower the staggered strength, the gap opens up again and the Chern numbers for the highest, middle, 
(a)

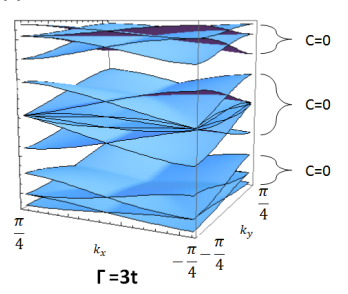

(b)

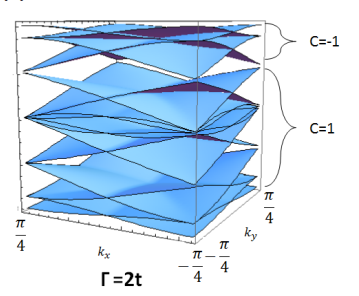

(c)

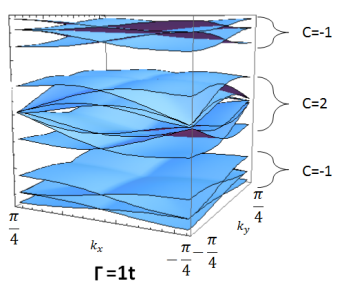

(d)

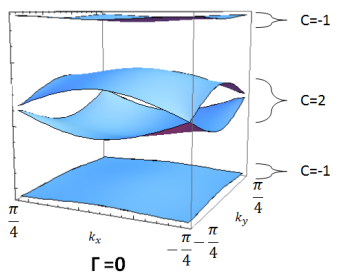

Figure B.1: RMFT energy spectrum as a function of staggered potential $\delta$ with Chern numbers for each band shown beside the figure. For $\Gamma>2 t$ the system is topologically trivial with the Chern number $C$ of the bands zero. At the transition point, the band gap closes and it becomes topologically non-trivial with $C=1$ for the lowest band. After passing the transition point, the gap opens again and the lowest band now possesses a Chern number of -1 . Notice that within this chosen reduced BZ, each of the four bands originating from the $2 \times 2$ modulation is folded into 4 sub-bands, producing a total of 16 bands.

and lowest bands become $-1,2$, and -1 , respectively. When the staggered number is equal to zero, the system is similar to the Harper-Hofstadter model with $\Phi=1 / 4$. Our results reveal a competition between the topological phase and the (induced) CDW, which has been experimentally realized by Aidelsburger et al. [100]. 
